Estimação em modelos funcionais com erro normais e repetições não balanceadas

\author{
Joan Neylo da Cruz Rodriguez
}

DISSERTAÇÃO APRESENTADA

$\mathrm{AO}$

INSTITUTO DE MATEMÁTICA E ESTATÍSTICA

DA

UNIVERSIDADE DE SÃO PAULO

PARA

OBTENÇÃO DO TÍTULO DE MESTRE

EM

CIÊNCIAS

\author{
Área de Concentração: Estatística \\ Orientador: Prof. Dr. Heleno Bolfarine
}

Durante a elaboração deste trabalho o autor recebeu auxílio financeiro do CNPq

São Paulo, abril de 2008. 


\title{
Estimação em modelos funcionais com erro normais e repetições não balanceadas
}

\author{
Este exemplar corresponde à redação \\ final da dissertação de mestrado, \\ devidamente corrigida e defendida por \\ Joan Neylo da Cruz Rodriguez e \\ aprovada pela comissão julgadora.
}

São Paulo, 29 de abril de 2008.

Banca Examinadora:

- Prof. Dr. Heleno Bolfarine (Presidente) - IME/USP

- $\operatorname{Prof}^{a}$. Dr ${ }^{a}$. Mônica Carneiro Sandoval - IME/USP

- Prof. Dr. Victor Hugo Lachos Davila - IMECC/UNICAMP 
Aos meus pais e meus irmãos. 
À minha preciosa esposa

Claudia, pelo incentivo, companherismo e acima de tudo, amor e respeito. Além de tudo isso, por significar que o amor pode suportar as distâncias e os obstáculos da vida. 


\section{Agradecimentos}

- Aos meus pais, Wilfredo e Luz (in memorian), por todo o carinho, motivação e apoio em todos os sentidos que sempre me deram.

- Ao meu orientador Prof. Dr. Heleno Bolfarine, por sua compreensão, dedicação e orientação, que de uma maneira clara e objetiva sempre me direcionou para um bom desenvolvimento desse trabalho.

- Aos companheiros da república: Christian Noriega, Ivan, Juan Carlos Cutipa que se tornaram minha família aqui em São Paulo.

- Aos amigos Lourdes, Juan Soto, Jannet Mercado, Jesus Mena, Karina Valdivia, Cristian Paz, Cristian Bayes, Pedro Fujita, Karina Preto, Fernando Zerecetti, Grazielle, Fernado Cristiano, Betsabé e Nubia pelo prazer de suas amizades, conversas e trocas de conhecimentos.

- À Jenny Niño, Zoraida, Marcia, Bruno e Henrry Javier pela amizade cultivada em todo momento.

- À mulher mais amada da face da terra Claudia, pelo apoio, preocupação e carinho nesta fase da minha vida.

- À Profa. Dra. Denice Aparecida Botter pela sua ajuda e amizade durante o mestrado.

- Aos meus professores no IME-USP. 
- Aos amigos que incentivaram minha vinda para o IME-USP, como a Profa. Rosario Bullon Cuadrado e o Prof. Oswaldo Ramos Chumpitas da Facudade de Ciencias Matematicas - UNMSM, ao Prof. Antonio Bravo e muitos outros.

- À banca examinadora, pelos comentários e pelas sugestões.

- Ao IME e à USP pela oportunidade de fazer meu Mestrado nesta Instituição, e ao CNPq pelo apoio financeiro.

- Ademais, gostaria de agradecer à todos aqueles, que colaboraram de uma forma ou de outra para a realização deste trabalho.

Finalmente, agradeço a Deus, por sua proteção, e por ter me dado força e vida ao longo de todo este período. 


\section{Resumo}

Esta dissertação compreende um estudo da eficiência de estimadores dos parâmetros no modelo funcional com erro nas variáveis, com repetições para contornar o problema de falta de identificação. Nela, discute-se os procedimentos baseados nos métodos de máxima verossimilhança e escore corrigido. As estimativas obtidas pelos dois métodos levam a resultados similares. 


\section{Abstract}

This work is concerned with a study on the efficiency of parameter estimates in the functional linear relashionship with constant variances. Lack of identification is resolved of by considering replications. Estimation is dealt with by using maximum likelihood and the corrected score approach. Comparisons between the approaches are illustrated by using simulated data. 


\section{Sumário}

Resumo vi

Abstract vii

Lista de Tabelas $\quad$ xi

1 Introdução 1

1.1 Organização da dissertação . . . . . . . . . . . . . . . . . . . . 3

2 Modelo Funcional Normal com Replicações 4

2.1 Introdução . . . . . . . . . . . . . . . . . . . . . . . . . . . 4

2.2 Modelo funcional normal com replicações . . . . . . . . . . . . . . . . 6

2.3 Estimação na presença de parâmetros incidentais . . . . . . . . . . 7

2.4 Estimação por máxima verossimilhança . . . . . . . . . . . . . . . 8

2.4 .1 Cálculos dos EMV . . . . . . . . . . . . . . . . . . 8 
2.4.2 Forma numérica dos estimadores para $(n=2) \ldots \ldots$

2.5 O Método Delta para $n=2 \ldots \ldots \ldots . \ldots . \ldots . \ldots 19$

2.6 Inferência usando verossimilhança perfilada . . . . . . . . . . . . . 23

2.6.1 Notações e resultados preliminares . . . . . . . . . . . . . . 23

2.6.2 Comportamento assintótico dos EMV . . . . . . . . . 25

2.6.3 Estimadores obtidos por Dorf e Gurland . . . . . . . . . . 28

2.6.4 Comparação dos estimadores de MV e Dorf e Gurland . . . . 30

$\begin{array}{lll}3 & \text { O Enfoque do escore corrigido } & 33\end{array}$

3.1 Introdução . . . . . . . . . . . . . . . . . . . . . . . . . . . . . 33

3.2 Descrição do método . . . . . . . . . . . . . . . . . . . . . . . 34

3.2 .1 Condições de regularidade . . . . . . . . . . . . . . 35

3.3 Aplicação do método com $\sigma_{u u}$ conhecido . . . . . . . . . . . . . . 36

3.3.1 Cálculos para encontrar estimador para a variância assintótica 40

3.4 Aplicação do método quando $\lambda=\frac{\sigma_{e e}}{\sigma_{u u}}$ conhecido . . . . . . . . . . . . 43

3.4.1 Cálculos para encontrar a matriz de covariâncias assintótica . 46

4 Comparação entre os métodos de estimação 48

4.1 Simulações . . . . . . . . . . . . . . . . . . . . . . . . . . . . 48

4.1.1 Resultados . . . . . . . . . . . . . . . . . . . 49 
5 Considerações finais $\quad 60$

A Condições de regularidade $\quad 62$

B O Método Delta $\quad 65$

C Programas em R 


\section{Lista de Tabelas}

2.1 Estimação dos parâmetros por MV, com tamanho de amostra $n=2$ com $r=\left(r_{1}, r_{2}\right)=(12,10)$ e $s=\left(s_{1}, s_{2}\right)=(10,8) \ldots \ldots \ldots$

2.2 Estimação dos parâmetros por MV, onde o ponto inicial é $x_{i}=\bar{X}_{i}+1$, obtendo-se a convergência na iteração 13. . . . . . . . . . . . 16

2.3 Estimação dos parâmetros por MV, com tamanho de amostra $n=2$ $\operatorname{com} r=\left(r_{1}, r_{2}\right)=(12,10)$ e $s=\left(s_{1}, s_{2}\right)=(10,8) \ldots \ldots 17$

2.4 Estimação dos parâmetros por MV, onde o ponto inicial é $x_{i}=\bar{X}_{i}+1$, obtendo-se a convergência na iteração 13. . . . . . . . . . . . 18

2.5 Vício e erro quadrático médio empírico usando 5000 amostras simuladas, com tamanho de amostra $n=3$, réplicas de $s=(8,10,15)$ e $r=(16,20,30) \ldots \ldots \ldots \ldots \ldots \ldots$

2.6 Vício e erro quadrático médio empírico usando 5000 amostras simuladas, com tamanho de amostra $n=3$, réplicas de $s=(30,40,60)$ e $r=(40,60,80)$. 
2.7 Vício e erro quadrático médio empírico usando 5000 amostras simuladas, com tamanho de amostra $n=15$, réplicas variando entre $\mathrm{s}=(6,9,12$, $15,18,21,24,21,18,15,12,9,6,9,12)$ e $\mathrm{r}=(11,14,17,20,23,26,29,26,23,20,17,14$,

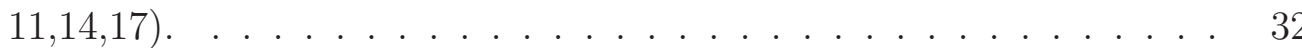

2.8 Vício e erro quadrático médio empírico usando 5000 amostras simuladas, com tamanho de amostra $n=30$, réplicas variando entre $\mathrm{s}=(6,9,12$, $15,18,21,24,21,18,15,12,9,6,9,12,15,18,21,24,21,18,15,12,9,6,9,12,15,18,21)$ e r $=(11,14,17,20,23,26,29,26,23,20,17,14,11,14,17,20,23,26,29,26,23,20,17$, $14,11,14,17,20,23,26) \ldots \ldots \ldots \ldots \ldots$

4.1 Vício e erro quadrático médio empírico, com tamanho de amostra $n=$ 3 , réplicas de valores $r=(16,20,30)$ e $s=(8,10,15) \ldots \ldots \ldots 1$

4.2 Vício e erro quadrático médio empírico, com tamanho de amostra $n=$ 3 , réplicas de valores $r=(16,20,30)$ e $s=(8,10,15) \ldots \ldots . . \ldots 52$

4.3 Vício e erro quadrático médio empírico, com tamanho de amostra $n=$ 3 , réplicas de valores $r=(16,20,30)$ e $s=(8,10,15) \ldots \ldots \ldots 3$

4.4 Vício e erro quadrático médio empírico, com tamanho de amostra $n=$ 15 , réplicas de valores $\mathrm{r}=(11,14,17,20,23,26,29,26,23,20,17,14,11,14,17)$ e $\mathrm{s}=(6,9,12,15,18,21,24,21,18,15,12,9,6,9,12) \ldots \ldots \ldots \ldots$

4.5 Vício e erro quadrático médio empírico, com tamanho de amostra $n=$ 15 , réplicas de valores $\mathrm{r}=(11,14,17,20,23,26,29,26,23,20,17,14,11,14,17)$ e $\mathrm{s}=(6,9,12,15,18,21,24,21,18,15,12,9,6,9,12)$. 
4.6 Vício e erro quadrático médio empírico, com tamanho de amostra $n=$ 15 , réplicas de valores $\mathrm{r}=(11,14,17,20,23,26,29,26,23,20,17,14,11,14,17)$ e $\mathrm{s}=(6,9,12,15,18,21,24,21,18,15,12,9,6,9,12) \ldots \ldots \ldots \ldots$

4.7 Vício e erro quadrático médio empírico, com tamanho de amostra $n=$ 30 , réplicas de valores $\mathrm{r}=(11,14,17,20,23,26,29,26,23,20,17,14,11,14,17$, $20,23,26,29,26,23,20,17,14,11,14,17,20,23,26)$ e $s=(6,9,12,15,18,21,24,21$, $18,15,12,9,6,9,12,15,18,21,24,21,18,15,12,9,6,9,12,15,18,21) \ldots \ldots . . .57$

4.8 Vício e erro quadrático médio empírico, com tamanho de amostra $n=$ 30 , réplicas de valores $\mathrm{r}=(11,14,17,20,23,26,29,26,23,20,17,14,11,14,17$, $20,23,26,29,26,23,20,17,14,11,14,17,20,23,26)$ e $s=(6,9,12,15,18,21,24,21$, $18,15,12,9,6,9,12,15,18,21,24,21,18,15,12,9,6,9,12,15,18,21) \ldots \ldots . . .58$

4.9 Vício e erro quadrático médio empírico, com tamanho de amostra $n=$ 30 , réplicas de valores $\mathrm{r}=(11,14,17,20,23,26,29,26,23,20,17,14,11,14,17$, $20,23,26,29,26,23,20,17,14,11,14,17,20,23,26)$ e s $=(6,9,12,15,18,21,24,21$, $18,15,12,9,6,9,12,15,18,21,24,21,18,15,12,9,6,9,12,15,18,21) \ldots \ldots . . .59$ 


\section{Capítulo 1}

\section{Introdução}

O objetivo desta dissertação é o desenvolvimento de estimadores para os parâmetros do modelo funcional com erro normais e com repetições. O estimador de máxima verossimilhança estudado por Hokama, Morettin, Bolfarine \& Galea-Rojas (2001) será comparado com um estimador definido pelo método do escore corrigido considerando as boas propriedades do método, como demonstrado em Gimenez \& Bolfarine (1997).

O modelo funcional é adotado porque não requer a especifição de uma distribuição para os parâmetros incidentais. E o uso de replicações não balanceadas não permite uma representação matricial na forma de uma equação de regressão linear.

A motivação do trabalho é usar replicações não balanceadas num estudo do modelo de regressão linear simples, $y_{i}=\alpha+\beta x_{i}+e_{i}$, para garantir uma abordagem factível sem suposições adicionais sobre as variâncias dos erros. Pode-se dar uma solução para a estimação dos parâmetros mediante o uso do método do escore corrigido. Para assegurar a identificabilidade do modelo mediante o método do escore corrigido usa-se suposições adicionais sobre as variâncias dos erros. 
O modelo funcional considera que " $x_{i}$ " não é observado e sem distribuição, portanto, os estimadores dos parâmetros definidos pelos métodos de máxima verossimilhança e do escore corrigido podem não ser consistentes. Para isto foram realizadas uma série de simulações, que serão apresentadas nos capítulos posteriores juntamente com os resultados obtidos.

Assim, o modelo considera $s_{i}$ e $r_{i}$ como os números de replicações para a unidade de observação "i", $y_{i}$ e $x_{i}$, respectivamente, sendo que estes valores são desconhecidos e serão observados com erro na variável resposta $Y_{i j}$, bem como na variável explicativa $X_{i k}$. Desta forma, considera-se a seguinte relação:

$$
Y_{i j}=\alpha+\beta x_{i}+e_{i j} \quad e \quad X_{i k}=x_{i}+u_{i k},
$$

ou seja, $y_{i}$ relaciona-se linearmente com $x_{i}$ sendo que $y_{i}=\alpha+\beta x_{i}$. Assim o modelo pode ser expressado da seguinte forma:

$$
Y_{i j}=y_{i}+e_{i j}, \quad X_{i k}=x_{i}+u_{i k}
$$

onde $e_{i j}$ e $u_{i k}$ são os erros que ocorrem ao medir estas variáveis. Para esta pesquisa considere-se que estes erros são não correlacionados e normais, ou seja

$$
\begin{gathered}
\left(\begin{array}{c}
e_{i j} \\
u_{i k}
\end{array}\right) \sim N_{2}\left[\left(\begin{array}{c}
0 \\
0
\end{array}\right),\left(\begin{array}{cc}
\sigma_{e e} & 0 \\
0 & \sigma_{u u}
\end{array}\right)\right], \\
\left(X_{i k}, Y_{i j}\right), \quad j=1, \ldots, s_{i}, \quad k=1, \ldots, r_{i} \quad e i=1, \ldots, n,
\end{gathered}
$$

onde,

- $Y_{i j}$ corresponde a $i$-ésima unidade de observação com a $j$-ésima réplica;

- $X_{i k}$ corresponde a $i$-ésima unidade de observação com a $k$-ésima réplica;

- $\alpha$ é o intercepto; 
- $\beta$ é o coeficiente angular ou a taxa de inclinação;

- $\sigma_{e e}$ é a variância adquirida ao medir $y$;

- $\sigma_{u u}$ é a variância adquirida ao medir $x$.

\subsection{Organização da dissertação}

A presente dissertação de mestrado está dividida em 5 capítulos e anexos. No segundo capítulo, apresenta-se o modelo funcional normal com replicações e as estimações dos parâmetros na presença de parâmetros incidentais mediante o método de máxima verossimilhança, onde destacam-se os resultados obtidos por Hokama et al. (2001) e que serão comparados com os obtidos por Dorf \& Gurland (1960).

No terceiro capítulo, apresenta-se a estimação dos parâmetros mediante o método de escore corrigido e as propriedades que oferece este estimador, supondo a variância dos erros de medida conhecida.

No quarto capítulo, apresenta-se uma aplicação baseada em dados simulados com certos valores fixados para os parâmetros, onde a forma de $x_{i}$ é conhecida, avaliando a precisão dos estimadores mediante o viés e o erro quadrático médio para os dois métodos utilizados; máxima verossimilhança "MV" e "escore corrigido".

Finalmente, no quinto capítulo apresentam-se as conclusões dos resultados obtidos neste trabalho. 


\section{Capítulo 2}

\section{Modelo Funcional Normal com Replicações}

\subsection{Introdução}

Neste capítulo estuda-se a estimação de máxima verossimilhança dos parâmetros estruturais na relação linear funcional com replicações. Esta abordagem é baseada em Mak (1982), onde resultados assintóticos gerais para estimação de máxima verossimilhança na presença de parâmetros incidentais são desenvolvidos para o caso sem replicações. Para o caso com replicações, os resultados são estabelecidos por Hokama et al. (2001) e Dorf \& Gurland (1960).

A estimação dos parâmetros estruturais por máxima verossimilhança, quando o número de parâmetros desconhecidos aumenta com o tamanho da amostra, apresenta problemas de identificação (ver Neyman \& Scott 1948); a matriz de covariância assintótica desses estimadores não coincide com o inverso da matriz de informação de Fisher (ver Patefield 1977 e 1978); sob a normalidade dos erros de medida, o logaritmo 
da função de verossimilhança é ilimitado, segundo Solari (1969) a raiz das equações da primeira derivada parcial em relação aos parâmetros estruturais é um ponto de cela e não de máximo, e Vilca-Labra, Arellano-Valle \& Bolfarine (1998) mostra que o valor de $\boldsymbol{\beta}$ que maximiza tal função não pertence à região do espaço paramétrico.

Considere $\sigma_{e e}$, a variância do erro ao observar a variável $y$ e $\sigma_{u u}$, a variância do erro ao observar a variável $x$ com relação linear $y=\alpha+\beta x$. Neste contexto são necessárias suposições adicionais, que tornam factível o estudo de inferência sob normalidade. Suposições comumente adotadas incluem:

- (i) $\sigma_{e e}$ e $\sigma_{u u}$ conhecidos (Barnett,1969, obtém estimadores de máxima verossimilhança de forma fechada).

- (ii) $\sigma_{e e}\left(\right.$ ou $\sigma_{u u}$ ) conhecida (veja Cheng \& Van Ness, 1991, entre outros).

- (iii) $\lambda=\sigma_{e e} / \sigma_{u u}$ conhecida (Gleser, 1985, entre outros).

Contudo, Mak (1982), sob um modelo bem geral, estabeleceu certas condições que garantem a consistência e a normalidade assintótica dos estimadores dos parâmetros estruturais (que incluem, em particular, o estimador de máxima verossimilhança $($ EMV $)$ ) obtidos via abordagem da verossimilhança usual, quando os parâmetros incidentais são primeiramente substituídos por estimativas (que podem depender de parâmetros estruturais). Essas condições foram aplicadas por Mak (1982), entre outros, ao modelo funcional univariado definido pela relação linear:

$$
y_{i}=\alpha+\beta x_{i}
$$

onde $x_{1}, x_{2}, \ldots, x_{n}$ são quantidades não aleatórias ou parâmetros incidentais e $\alpha$ e $\beta$ são parâmetros estruturais desconhecidos. Os pares $x_{i}, y_{i}$ são os verdadeiros valores das variáveis explanatórias e das respostas não observáveis ou observadas com erro. 
Observa-se os pares $\left(X_{i}, Y_{i}\right)$, de modo que, sob normalidade, pode-se escrever:

$$
\begin{gathered}
X_{i}=x_{i}+u_{i}, \quad Y_{i}=y_{i}+e_{i} \\
\left(\begin{array}{c}
e_{i} \\
u_{i}
\end{array}\right) \sim N_{2}\left[\left(\begin{array}{l}
0 \\
0
\end{array}\right),\left(\begin{array}{cc}
\sigma_{e e} & 0 \\
0 & \sigma_{u u}
\end{array}\right)\right], \quad i=1,2, \ldots, n .
\end{gathered}
$$

Note que Mak (1982) usa a suposição (iii) para obter os estimadores de máxima verossimilhança dos parâmetros estruturais do modelo.

O objetivo é estudar o modelo funcional com erro nas variáveis com replicações, que é uma maneira de garantir uma abordagem factível sem suposições adicionais sobre as variâncias dos erros. Assim, considerando $s_{i}$ e $r_{i}$ números de replicações para $y_{i}$ e $x_{i}$, respectivamente, reescreve-se o modelo acima como:

$$
\begin{gathered}
Y_{i j}=y_{i}+e_{i j}, \quad X_{i k}=x_{i}+u_{i k}, \\
\left(\begin{array}{c}
e_{i j} \\
u_{i k}
\end{array}\right) \sim N_{2}\left[\left(\begin{array}{l}
0 \\
0
\end{array}\right),\left(\begin{array}{cc}
\sigma_{e e} & 0 \\
0 & \sigma_{u u}
\end{array}\right)\right], \\
j=1, \ldots, s_{i}, \quad k=1, \ldots, r_{i} \text { e } i=1, \ldots, n .
\end{gathered}
$$

O vetor de parâmetros desconhecidos é $\boldsymbol{\theta}=\left(\alpha, \beta, \sigma_{e e}, \sigma_{u u}, x_{1}, x_{2}, \ldots, x_{n}\right)$.

\subsection{Modelo funcional normal com replicações}

Neste caso, reescreve-se o modelo (2.2) como escrito em (2.3). Portanto, o termo não constante no logaritmo da função de verossimilhança correspondente aos dados observados na amostra $\left(X_{i k}, Y_{i j}\right), \operatorname{com} j=1, \ldots, s_{i}, k=1, \ldots, r_{i}$ e $i=1, \ldots, n$, é dado por:

$$
\ell \propto-\frac{1}{2} \sum_{i=1}^{n}\left[s_{i} \log \left(\sigma_{e e}\right)+r_{i} \log \left(\sigma_{u u}\right)\right]-\frac{1}{2}\left\{\sigma_{u u}^{-1} \sum_{i=1}^{n} \sum_{k=1}^{r_{i}}\left(X_{i k}-x_{i}\right)^{2}\right.
$$




$$
\left.+\sigma_{e e}^{-1} \sum_{i=1}^{n} \sum_{j=1}^{s_{i}}\left(Y_{i j}-\alpha-\beta x_{i}\right)^{2}\right\}
$$

onde $\alpha, \beta, \sigma_{e e}$ e $\sigma_{u u}$ são os parâmetros estruturais e $x_{i}, i=1, \ldots, n$, são os parâmetros incidentais. Note que este modelo é similar ao modelo proposto por Dorf \& Gurland (1960), onde são sugeridos estimadores baseados em análise de variância. A abordagem de máxima verossimilhança é investigada em Hokama et al. (2001), na qual estudos de eficiência são considerados. Estes autores também implementaram um algoritmo tipo EM, como considerado em Kimura (1992). Mais especificamente, têm-se:

- Considerando a razão constante entre os números de replicações $\left(r_{i} / s_{i}\right)$, o estimador de $\beta$ é obtido resolvendo-se uma equação de quarto grau. Maiores simplicações ocorrem se $r_{i}=r$ e $s_{i}=s$ ou no caso em que $r_{i}=r$ e $s_{i}=1$, isto é, apenas $x_{i}$ é replicado, constituindo-se em situações comuns em casos práticos (Dorff \& Gurland, 1960).

- Em Hokama et al. (2001) são establecidas propriedades dos EMVs dos parâmetros incidentais e estruturais, demonstrando que os estimadores de máxima verossimilhança de $\alpha$ e $\beta$ são consistentes. Os estimadores de $\sigma_{e e}$ e $\sigma_{u u}$ não são consistentes, mas uma correção adequada os tornam e suas respectivas variâncias assintóticas podem ser obtidas.

\subsection{Estimação na presença de parâmetros inciden- tais}

Sabe-se que em modelos com variáveis aleatórias independentes e identicamente distribuídas (iid) e sob condições de regularidade (Sen \& Singer, 1993), os EMV são 
consistentes e assintoticamente normais (AN). A matriz de covariância assintótica é dada pela inversa da matriz de informação de Fischer. Em muitas situações, no entanto, a "suposição" de observações iid não é satisfeita como, por exemplo, no modelo funcional definido em (2.2). Sob o modelo (2.3) e usando propriedades da distribuição normal multivariada, pode-se mostrar que

$$
\begin{aligned}
& \left(\begin{array}{c}
X_{i j} \\
Y_{i k}
\end{array}\right) \sim N_{2}\left[\left(\begin{array}{c}
x_{i} \\
\alpha+\beta x_{i}
\end{array}\right),\left(\begin{array}{cc}
\sigma_{e e} & 0 \\
0 & \sigma_{u u}
\end{array}\right)\right], \\
& j=1, \ldots, s_{i}, \quad k=1, \ldots, r_{i} \text { e } i=1, \ldots, n .
\end{aligned}
$$

\subsection{Estimação por máxima verossimilhança}

A estimação dos parâmetros por MV na presença de parâmetros incidentais onde o número de parâmetros desconhecidos cresce concomitantemente com o tamanho da amostra, a estimação dos parâmetros tem justificativa menos óbvia. No modelo (2.2), os estimadores de MV são inconsistentes e sua matriz de covariâncias assintóticas não é dada, necessariamente, pela inversa da matriz de informação de Fischer (ver Patefield, 1977 e Fuller, 1987).

Usando a mesma notação de Mak (1982), que propõe um método de estimação que inclui o método de máxima verossimilhança, considera-se, a seguir, a estimação por máxima verossimilhança para os parâmetros do modelo descrito em (2.3).

\subsubsection{Cálculos dos EMV}

Os EMV do vetor de parâmetros $\theta$ são obtidos derivando o logaritmo da função de verossimilhança em relação a $\beta, \alpha, \sigma_{e e}, \sigma_{u u}$ e $x_{i}, i=1, \ldots, n$. 
Derivando o logaritmo da função de verossimilhança dado em (2.4) em relação a $\beta$ e igulando a zero, temos que

$$
\sum_{i=1}^{n} \sum_{j=1}^{s_{i}}\left(x_{i} Y_{i j}-\widehat{\alpha} x_{i}-\widehat{\beta} x_{i}^{2}\right)=0 .
$$

Logo,

$$
\sum_{i=1}^{n} \sum_{j=1}^{s_{i}} x_{i} Y_{i j}-\widehat{\alpha} \sum_{i=1}^{n} \sum_{j=1}^{s_{i}} x_{i}=\widehat{\beta} \sum_{i=1}^{n} \sum_{j=1}^{s_{i}} x_{i}^{2}
$$

ou seja,

$$
\sum_{i=1}^{n} \sum_{j=1}^{s_{i}} x_{i} Y_{i j}-\widehat{\alpha} \sum_{i=1}^{n} s_{i} x_{i}=\widehat{\beta} \sum_{i=1}^{n} s_{i} x_{i}^{2} .
$$

Derivando $\ell$ em relação a $\alpha$, e igualando a zero segue que

$$
\sum_{i=1}^{n} \sum_{j=1}^{s_{i}}\left(Y_{i j}-\widehat{\alpha}-\widehat{\beta} x_{i}\right)=0
$$

Logo,

$$
\sum_{i=1}^{n} \sum_{j=1}^{s_{i}} Y_{i j}-\widehat{\beta} \sum_{i=1}^{n} s_{i} x_{i}=\widehat{\alpha} \sum_{i=1}^{n} s_{i}
$$

ou seja,

$$
\widehat{\alpha}=\frac{\sum_{i=1}^{n} \sum_{j=1}^{s_{i}} Y_{i j}-\widehat{\beta} \sum_{i=1}^{n} s_{i} x_{i}}{\sum_{i=1}^{n} s_{i}} .
$$


Dado que $\sum_{i=1}^{n} s_{i}=s$ e $\sum_{i=1}^{n} r_{i}=r$, substituindo (2.7) em (2.6) tem-se que:

$$
\sum_{i=1}^{n} \sum_{j=1}^{s_{i}} x_{i} Y_{i j}-\left(\frac{\sum_{i=1}^{n} \sum_{j=1}^{s_{i}} Y_{i j}-\widehat{\beta} \sum_{i=1}^{n} s_{i} x_{i}}{s}\right) \sum_{i=1}^{n} s_{i} x_{i}=\widehat{\beta} \sum_{i=1}^{n} s_{i} x_{i}^{2}
$$

ou seja,

$$
\begin{aligned}
& \qquad \sum_{i=1}^{n} \sum_{j=1}^{s_{i}} x_{i} Y_{i j}-\frac{\sum_{i=1}^{n} s_{i} \bar{Y}_{i}}{s} \sum_{i=1}^{n} s_{i} x_{i}+\frac{\widehat{\beta}}{s}\left(\sum_{i=1}^{n} s_{i} x_{i}\right)^{2}=\widehat{\beta} \sum_{i=1}^{n} s_{i} x_{i}^{2} . \\
& \text { Em que } \quad \frac{\sum_{j=1}^{s_{i}} Y_{i j}}{s_{i}}=\bar{Y}_{i} \quad \text { e } \quad \frac{\sum_{k=1}^{r_{i}} X_{i k}}{r_{i}}=\bar{X}_{i} .
\end{aligned}
$$

Portanto,

$$
\widehat{\beta}=\frac{\sum_{i=1}^{n} \sum_{j=1}^{s_{i}} x_{i} Y_{i j}-\sum_{i=1}^{n} \frac{s_{i} \bar{Y}_{i}}{s} \sum_{i=1}^{n} s_{i} x_{i}}{\sum_{i=1}^{n} s_{i} x_{i}^{2}-\frac{1}{s}\left(\sum_{i=1}^{n} s_{i} x_{i}\right)^{2}} .
$$

Substituindo (2.8) em (2.7), temos

$$
\sum_{j=1}^{s_{i}} Y_{i j}-\left(\frac{\sum_{i=1}^{n} \sum_{j=1}^{s_{i}} x_{i} Y_{i j}-\sum_{i=1}^{n} \frac{s_{i} \bar{Y}_{i}}{s} \sum_{i=1}^{n} s_{i} x_{i}}{\sum_{i=1}^{n} s_{i} x_{i}^{2}-\frac{1}{s}\left(\sum_{i=1}^{n} s_{i} x_{i}\right)^{2}}\right) \sum_{i=1}^{n} s_{i} x_{i}=s \widehat{\alpha},
$$

resultando em

$$
\widehat{\alpha}=\sum_{i=1}^{n} \sum_{j=1}^{s_{i}} \frac{Y_{i j}}{s}-\widehat{\beta} \sum_{i=1}^{n} \frac{s_{i} x_{i}}{s}
$$


Resolvendo

$$
\frac{\partial \ell}{\partial \sigma_{e e}}=0
$$

temos

$$
\left(-\frac{1}{2}\right) \sum_{i=1}^{n} \frac{s_{i}}{\widehat{\sigma}_{e e}}-\frac{1}{2} \times(-1) \times\left(\frac{-1}{\widehat{\sigma}_{e e}^{2}}\right) \sum_{i=1}^{n} \sum_{j=1}^{s_{i}}\left(Y_{i j}-\widehat{\alpha}-\widehat{\beta} x_{i}\right)^{2}=0
$$

ou seja,

$$
\widehat{\sigma}_{e e}=\frac{\sum_{i=1}^{n} \sum_{j=1}^{s_{i}}\left(Y_{i j}-\widehat{\alpha}-\widehat{\beta} x_{i}\right)^{2}}{s} .
$$

De maneira similar, fazendo

$$
\frac{\partial \ell}{\partial \sigma_{u u}}=0
$$

temos

$$
-\frac{1}{2} \sum_{i=1}^{n} \frac{r_{i}}{\widehat{\sigma}_{u u}}-\frac{1}{2} \times-1 \times \frac{1}{\widehat{\sigma}_{u u}^{2}} \sum_{i=1}^{n} \sum_{k=1}^{r_{i}}\left(X_{i k}-\widehat{x}_{i}\right)^{2}=0 .
$$

quando $\sum_{i=1}^{n} r_{i}=r$, resulta em

$$
\widehat{\sigma}_{u u}=\frac{\sum_{i=1}^{n} \sum_{k=1}^{r_{i}}\left(X_{i k}-\widehat{x}_{i}\right)^{2}}{r} .
$$

Finalmente, derivando $\ell$ em relação a $x_{i}$ e igualando a zero, obtemos

$$
-\frac{1}{2}\left[\widehat{\sigma}_{u u}^{-1} \sum_{k=1}^{r_{i}}\left(X_{i k}-\widehat{x}_{i}\right) \times-2+\widehat{\sigma}_{e e}^{-1} \sum_{j=1}^{s_{i}}\left(Y_{i j}-\widehat{\alpha}-\widehat{\beta} \widehat{x}_{i}\right) \times-2 \widehat{\beta}\right]=0
$$

ou seja,

$$
\widehat{\sigma}_{u u}^{-1}\left(\sum_{k=1}^{r_{i}} X_{i k}-r_{i} \widehat{x}_{i}\right)+\widehat{\beta} \widehat{\sigma}_{e e}^{-1}\left(\sum_{j=1}^{s_{i}} Y_{i j}-s_{i} \widehat{\alpha}-\widehat{\beta} s_{i} \widehat{x}_{i}\right)=0
$$


e, portanto,

$$
\widehat{x_{i}}=\frac{{\widehat{\sigma_{u u}}}^{-1} \sum_{k=1}^{r_{i}} X_{i k}+\widehat{\beta} \widehat{\sigma}_{e e}^{-1}\left(\sum_{j=1}^{s_{i}} Y_{i j}-s_{i} \widehat{\alpha}\right)}{r_{i} \widehat{\sigma}_{u u}^{-1}+s_{i} \widehat{\beta}^{2}{\widehat{\sigma_{e e}}}^{-1}},
$$

assim,

$$
\widehat{x}_{i}=\bar{X}_{i}\left[\frac{r_{i} \widehat{\sigma}_{u u}^{-1}+s_{i} \widehat{\beta}^{2} \widehat{\sigma}_{e e}^{-1}\left(\frac{\bar{Y}_{i}-\widehat{\alpha}}{\widehat{\beta}_{i}}\right)}{r_{i} \widehat{\sigma}_{u u}^{-1}+s_{i} \widehat{\beta}^{2} \widehat{\sigma}_{e e}^{-1}}\right] \text {, para } i=1, \ldots, n
$$

Assim, para efeitos práticos, nas equações anteriores $(2.8),(2.9),(2.10),(2.11)$ e (2.13) se realizará um processo de convergência iterativa para obter as estimativas dos parâmetros estruturais e incidentais. 


\subsubsection{Forma numérica dos estimadores para $(n=2)$}

Mostra-se, nesta seção, que para amostras de tamanho dois $(n=2)$, com erro nas variáveis no modelo funcional normal com replicações (2.3), os estimadores têm forma fechada, possível de ser demonstrado numericamente.

A seguir, é proposto um estimador consistente para $n=2$, sugerido a partir de $\widehat{x}_{1}=\bar{X}_{1}$ e $\widehat{x}_{2}=\bar{X}_{2}$. Assim, substituindo na equação (2.8) obteve-se o seguinte:

$$
\begin{aligned}
\widehat{\beta} & =\frac{s_{1} \bar{X}_{1} \bar{Y}_{1}+s_{2} \bar{X}_{2} \bar{Y}_{2}-\left(s_{1} \bar{Y}_{1}+s_{2} \bar{Y}_{2}\right)\left(s_{1} \bar{X}_{1}+s_{2} \bar{X}_{2}\right) / s}{s_{1} \bar{X}_{1}^{2}+s_{2} \bar{X}_{2}^{2}-\left(s_{1} \bar{X}_{1}+s_{2} \bar{X}_{2}\right)^{2} / s} \\
& =\frac{\left[\left(s_{1}+s_{2}\right)\left(s_{1} \bar{X}_{1} \bar{Y}_{1}+s_{2} \bar{X}_{2} \bar{Y}_{2}\right)-\left(s_{1} \bar{Y}_{1}+s_{2} \bar{Y}_{2}\right)\left(s_{1} \bar{X}_{1}+s_{2} \bar{X}_{2}\right)\right] / s}{s_{1} \bar{X}_{1}^{2}+s_{2} \bar{X}_{2}^{2}-\left(s_{1} \bar{X}_{1}+s_{2} \bar{X}_{2}\right)^{2} / s} \\
& =\frac{s_{1} s_{2} \bar{X}_{2} \bar{Y}_{2}+s_{1} s_{2} \bar{X}_{1} \bar{Y}_{1}+s_{2}^{2} \bar{X}_{2} \bar{Y}_{2}-s_{1} s_{2} \bar{Y}_{1} \bar{X}_{2}-s_{1} s_{2} \bar{X}_{1} \bar{Y}_{2}-s_{2}^{2} \bar{X}_{2} \bar{Y}_{2}}{s_{1}^{2} \bar{X}_{1}^{2}+s_{1} s_{2} \bar{X}_{2}^{2}+s_{1} s_{2} \bar{X}_{1}^{2}+s_{2}^{2} \bar{X}_{2}^{2}-\left[s_{1}^{2} \bar{X}_{1}^{2}+2 s_{1} s_{2} \bar{X}_{1} \bar{X}_{2}+s_{2}^{2} \bar{X}_{2}^{2}\right]} \\
& =\frac{s_{1} s_{2}\left[\bar{X}_{2} \bar{Y}_{2}+\bar{X}_{1} \bar{Y}_{1}-\bar{Y}_{1} \bar{X}_{2}-\bar{X}_{1} \bar{Y}_{2}\right]}{s_{1} s_{2}\left[\bar{X}_{2}^{2}+\bar{X}_{1}^{2}-2 \bar{X}_{1} \bar{X}_{2}\right]} \\
& =\frac{\bar{Y}_{1}\left(\bar{X}_{1}-\bar{X}_{2}\right)-\bar{Y}_{2}\left(\bar{X}_{1}-\bar{X}_{2}\right)}{\left(\bar{X}_{1}-\bar{X}_{2}\right)^{2}} \\
& =\frac{\left(\bar{X}_{1}-\bar{X}_{2}\right)\left(\bar{Y}_{1}-\bar{Y}_{2}\right)}{\left(\bar{X}_{1}-\bar{X}_{2}\right)^{2}}
\end{aligned}
$$

ou seja,

$$
\widehat{\beta}=\frac{\bar{Y}_{1}-\bar{Y}_{2}}{\bar{X}_{1}-\bar{X}_{2}}
$$

Onde o estimador encontrado " $\widehat{\beta}$ " para $n=2$, denotaremos por $\widehat{\beta}_{J}=\frac{\bar{Y}_{1}-\bar{Y}_{2}}{\bar{X}_{1}-\bar{X}_{2}}$, que é consistente para $s_{1}, s_{2}, r_{1}, r_{2} \rightarrow \infty$, ou seja,

$$
\widehat{\beta}_{J} \stackrel{P}{\rightarrow} \frac{\alpha+\beta x_{1}-\alpha-\beta x_{2}}{x_{1}-x_{2}}=\beta,
$$


onde " $\stackrel{P}{\rightarrow}$ " significa convergência em probabilidade, pois, $\bar{X}_{1} \stackrel{P}{\rightarrow} x_{1}$ e $\bar{X}_{2} \stackrel{P}{\rightarrow} x_{2}$, de modo que $\bar{Y}_{1} \stackrel{P}{\rightarrow} \alpha+\beta x_{1}$ e $\bar{Y}_{2} \stackrel{P}{\rightarrow} \alpha+\beta x_{2}$.

$$
\bar{Y}_{1}=\alpha+\beta x_{1}+\bar{e}_{1}
$$

e como $E\left[\bar{e}_{1}\right]=0$ e $\operatorname{Var}\left(\bar{e}_{1}\right)=\frac{1}{s_{1}^{2}} \sum_{j=1}^{s_{1}} \operatorname{Var}\left(e_{1 j}\right)=\frac{\sigma_{e e}}{s_{1}}$, então

$$
\bar{Y}_{1} \stackrel{P}{\rightarrow} \alpha+\beta x_{1}
$$

Idem para $\bar{Y}_{2}$.

Além disso, foi verificado através de estudos de simulação que, $\widehat{\beta}_{J}$ é o estimador de máxima verossimilhança de $\beta$, neste caso particular. Contudo, não encontrou-se uma justificativa teórica formal para esta situação.

Assim, fez-se uma simulação numérica iterativa para os estimadores de máxima verossimilhança obtidos em $(2.8),(2.9),(2.10),(2.11)$ e (2.13).

\section{O algoritmo utilizado pode ser escrito da seguinte forma:}

1. Com valores iniciais para $\widehat{x}_{i}^{0}$ quando " $n=2$ ", inicializa-se em $\widehat{x}_{1}^{0}=\bar{X}_{1}$ e $\widehat{x}_{2}^{0}=\bar{X}_{2} ;$ inicializar $j=1$,

2. substituir $\widehat{x}_{i}^{j-1}$ na equação $(2.8)$, e obter $\widehat{\beta}^{j}$,

3. substituir $\widehat{\beta}^{j}$ na equação (2.9), e obter $\widehat{\alpha}^{j}$,

4. substituir (2.), (3.) na equação (2.10), e obter $\widehat{\sigma}_{e e}^{j}$,

5. substituir (2.) na equação (2.11), e obter $\widehat{\sigma}_{u u}^{j}$ 
6. substituir (2.), (3.), (4.) e (5.) na equação (2.13), dada pela equação $\widehat{x}_{i}^{j}$,

7. fazer $j=j+1$ e repetir a partir do passo (2.).

\section{Resultados das simulações do algoritmo utilizado}

Detalha-se a seguir passo a passo o procedimento do algoritmo que foi programado utilizando o software estatístico R Development Core Team (2006). Nele demonstrase numericamente que o parâmetro incidental tem uma solução numérica fechada, sendo que o verdadeiro valor do parâmetro incidental é estimado pela média do grupo replicado. Pode-se observar na Tabela 2.1 as simulações realizadas. O programa utilizado encontra-se em anexo.

Tabela 2.1: Estimação dos parâmetros por MV, com tamanho de amostra $n=2$ com $r=\left(r_{1}, r_{2}\right)=(12,10)$ e $s=\left(s_{1}, s_{2}\right)=(10,8)$.

\begin{tabular}{c|c|c|c|c|cc}
\hline iteração & \multicolumn{4}{|c|}{ parâmetros estruturais } & \multicolumn{2}{c}{ parâmetro incidental } \\
\hline$j$ & $\widehat{\alpha}$ & $\widehat{\beta}$ & $\widehat{\sigma}_{e e}$ & $\widehat{\sigma}_{u u}$ & $x_{1}$ & $x_{2}$ \\
\hline$j=0$ & 0,8789684 & 1,003142 & 2,011027 & 0,9234677 & 10,17963 & 6,703495 \\
$j=1$ & 0,8789684 & 1,003142 & 2,011027 & 0,9234677 & 10,17963 & 6,703495 \\
$j=2$ & 0,8789684 & 1,003142 & 2,011027 & 0,9234677 & 10,17963 & 6,703495 \\
$j=3$ & 0,8789684 & 1,003142 & 2,011027 & 0,9234677 & 10,17963 & 6,703495 \\
$j=4$ & 0,8789684 & 1,003142 & 2,011027 & 0,9234677 & 10,17963 & 6,703495 \\
\hline & & $\widehat{\beta}_{J}=\frac{\bar{Y}_{1}-\bar{Y}_{2}}{\bar{X}_{1}-\bar{X}_{2}}$ & & & $\bar{X}_{1}=10,179629$ & $\bar{X}_{2}=6,703495$ \\
& & $\widehat{\beta}_{J}=1,003142$ & & $\bar{Y}_{1}=11,090579$ & $\bar{Y}_{2}=7,603524$ \\
\hline \hline
\end{tabular}

Os valores verdadeiros utilizados na simulação foram: $\alpha=1, \beta=1, \sigma_{e e}=3$, $\sigma_{u u}=1$ e $x_{i} \sim N\left(\mu_{x} ; \sigma_{x x}\right), \operatorname{com} \mu_{x}=10$ e $\sigma_{x x}=3$. 
Com qualquer outro ponto inicial, por exemplo, $x_{1}=\bar{X}_{1}+1$ e $x_{2}=\bar{X}_{2}+1$, sempre acontecerá que $\widehat{x}_{i} \rightarrow \bar{X}_{i}$ para $n=2$, como é observado na Tabela 2.2. Esta simulação foi realizada com os mesmos parâmetros listados na Tabela 2.1.

Tabela 2.2: Estimação dos parâmetros por MV, onde o ponto inicial é $x_{i}=\bar{X}_{i}+1$, obtendo-se a convergência na iteração 13.

\begin{tabular}{c|c|c|c|c|cc}
\hline iteração & \multicolumn{4}{|c|}{ parâmetros estruturais } & \multicolumn{2}{c}{ parâmetro incidental } \\
\hline$j$ & $\widehat{\alpha}$ & $\widehat{\beta}$ & $\widehat{\sigma}_{e e}$ & $\widehat{\sigma}_{u u}$ & $x_{1}$ & $x_{2}$ \\
\hline$j=1$ & $-0,1241733$ & 1,003142 & 2,011027 & 1,9234677 & 10,62471 & 7,138518 \\
$j=2$ & 0,4632395 & 1,000247 & 2,011027 & 1,1175414 & 10,32055 & 6,837419 \\
$j=3$ & 0,7584038 & 1,001126 & 2,011027 & 0,9424525 & 10,21927 & 6,740073 \\
$\vdots$ & $\vdots$ & $\vdots$ & $\vdots$ & $\vdots$ & $\vdots$ & $\vdots$ \\
$j=11$ & 0,8789670 & 1,003141 & 2,011027 & 0,9234677 & 10,17963 & 6,703496 \\
$j=12$ & 0,8789681 & 1,003142 & 2,011027 & 0,9234677 & 10,17963 & 6,703495 \\
$j=13$ & 0,8789684 & 1,003142 & 2,011027 & 0,9234677 & 10,17963 & 6,703495 \\
$j=14$ & 0,8789684 & 1,003142 & 2,011027 & 0,9234677 & 10,17963 & 6,703495 \\
\hline & & $\widehat{\beta}_{J}=\frac{\bar{Y}_{1}-\bar{Y}_{2}}{\bar{X}_{1}-\bar{X}_{2}}$ & & & $\bar{X}_{1}=10,179629$ & $\bar{X}_{2}=6,703495$ \\
& & $\widehat{\beta}_{J}=1,003142$ & & & $\bar{Y}_{1}=11,090579$ & $\bar{Y}_{2}=7,603524$ \\
\hline
\end{tabular}


Observa-se nas Tabelas 2.1 e 2.2 que os valores obtidos como estimadores de $x_{1}$ e $x_{2}$ foram, respectivamente, $\widehat{x}_{1}=10,17963$, que é igual a $\bar{X}_{1}=10,179629 \mathrm{e}$ $\widehat{x}_{2}=6,703495$ que é igual a $\bar{X}_{2}=6,703495$. Além disso, observa-se que o estimador de $\beta$ é $\widehat{\beta}_{J}=1,003142$. Isto acontece para qualquer outra distribuição de $x$, como é mostrado nas Tabelas 2.3 e 2.4 .

Tabela 2.3: Estimação dos parâmetros por MV, com tamanho de amostra $n=2$ com $r=\left(r_{1}, r_{2}\right)=(12,10)$ e $s=\left(s_{1}, s_{2}\right)=(10,8)$.

\begin{tabular}{c|c|c|c|c|cc}
\hline iteração & \multicolumn{4}{|c|}{ parâmetros estruturais } & \multicolumn{2}{c}{ parâmetro incidental } \\
\hline$j$ & $\widehat{\alpha}$ & $\widehat{\beta}$ & $\widehat{\sigma}_{e e}$ & $\widehat{\sigma}_{u u}$ & $x_{1}$ & $x_{2}$ \\
\hline$j=0$ & 1,617614 & 1,003923 & 2,126941 & 1,064297 & 13,68794 & 5,446632 \\
$j=1$ & 1,617614 & 1,003923 & 2,126941 & 1,064297 & 13,68794 & 5,446632 \\
$j=2$ & 1,617614 & 1,003923 & 2,126941 & 1,064297 & 13,68794 & 5,446632 \\
$j=3$ & 1,617614 & 1,003923 & 2,126941 & 1,064297 & 13,68794 & 5,446632 \\
$j=4$ & 1,617614 & 1,003923 & 2,126941 & 1,064297 & 13,68794 & 5,446632 \\
\hline & & $\widehat{\beta}_{J}=\frac{\bar{Y}_{1}-\bar{Y}_{2}}{\bar{X}_{1}-\bar{X}_{2}}$ & & & $\bar{X}_{1}=13,687944$ & $\bar{X}_{2}=5,446632$ \\
& & $\widehat{\beta}_{J}=1,003923$ & & $\bar{Y}_{1}=15,359257$ & $\bar{Y}_{2}=7,085614$ \\
& & $\widehat{\beta}_{J}=\widehat{\beta}$ & & & \\
\hline \hline
\end{tabular}

Os valores verdadeiros utilizados na simulação foram: $\alpha=1, \beta=1, \sigma_{e e}=3$, $\sigma_{u u}=1$ e $x_{i} \sim U(a, b), \operatorname{com} a=5$ e $b=15$. 
Tabela 2.4: Estimação dos parâmetros por MV, onde o ponto inicial é $x_{i}=\bar{X}_{i}+1$, obtendo-se a convergência na iteração 13.

\begin{tabular}{c|c|c|c|c|cc}
\hline iteração & \multicolumn{3}{|c|}{ parâmetros estruturais } & \multicolumn{2}{c}{ parâmetro incidental } \\
\hline$j$ & $\widehat{\alpha}$ & $\widehat{\beta}$ & $\widehat{\sigma}_{e e}$ & $\widehat{\sigma}_{u u}$ & $x_{1}$ & $x_{2}$ \\
\hline$j=1$ & 0,613691 & 1,003923 & 2,126941 & 2,064297 & 14,13702 & 7,138518 \\
$j=2$ & 1,184105 & 1,002697 & 2,126941 & 1,261902 & 13,83705 & 6,837419 \\
$j=3$ & 1,480201 & 1,003035 & 2,126941 & 1,085566 & 13,73263 & 6,740073 \\
$\vdots$ & $\vdots$ & $\vdots$ & $\vdots$ & $\vdots$ & $\vdots$ & $\vdots$ \\
$j=11$ & 1,617609 & 1,003923 & 2,126941 & 1,064297 & 13,68795 & 5,446634 \\
$j=12$ & 1,617613 & 1,003923 & 2,126941 & 1,064297 & 13,68795 & 5,588449 \\
$j=13$ & 1,617614 & 1,003923 & 2,126941 & 1,064297 & 13,68794 & 5,446632 \\
$j=14$ & 1,617614 & 1,003923 & 2,126941 & 1,064297 & 13,68794 & 5,446632 \\
\hline & & $\widehat{\beta}_{J}=\frac{\bar{Y}_{1}-\bar{Y}_{2}}{\bar{X}_{1}-\bar{X}_{2}}$ & & & $\bar{X}_{1}=13,687944$ & $\bar{X}_{2}=5,446632$ \\
& & $\widehat{\beta}_{J}=1,003923$ & & & $\bar{Y}_{1}=15,359257$ & $\bar{Y}_{2}=7,085614$ \\
& & $\widehat{\beta}_{J}=\widehat{\beta}$ & & & & \\
\hline
\end{tabular}

Observa-se que para qualquer $x_{1}=\bar{X}_{1}+1$ e $x_{2}=\bar{X}_{2}+1$, sempre acontecerá que $\widehat{x}_{i} \rightarrow \bar{X}_{i}$ para $n=2$, como é observado na Tabela 2.4. Esta simulação foi realizada com os mesmos parâmetros listados na Tabela 2.3. 


\subsection{O Método Delta para $n=2$}

Nesta seção obtemos aproximações para a variância assintótica do E.M.V. de $\beta$ para $n=2$. Mostramos que um estimador consistente para $\beta$ é dado por $\widehat{\beta}_{J}=\frac{\bar{Y}_{1}-\bar{Y}_{2}}{\bar{X}_{1}-\bar{X}_{2}}$.

Como

$$
Y_{1 j}=\alpha+\beta x_{1}+e_{1 j} \quad, j=1,2, \ldots, s_{1},
$$

com

$$
e_{1 j} \sim N\left(0 ; \sigma_{e e}\right) \text { e } e_{1 j}, e_{1 j^{\prime}} \text { independentes }
$$

temos que,

$$
Y_{1 j} \mid x_{1} \sim N\left(\alpha+\beta x_{1} ; \sigma_{e e}\right) \quad, \quad j=1,2, \ldots, s_{1},
$$

de modo que

$$
\bar{Y}_{1}=\sum_{j=1}^{s_{1}} \frac{Y_{1 j}}{s_{1}} \sim N\left(\alpha+\beta x_{1} ; \frac{\sigma_{e e}}{s_{1}}\right), \quad j=1,2, \ldots, s_{1} .
$$

De maneira análoga, temos

$$
Y_{2 j}=\alpha+\beta x_{2}+e_{2 j} \quad, j=1,2, \ldots, s_{2},
$$

com

$$
e_{2 j} \sim N\left(0 ; \sigma_{e e}\right) \text { e } e_{2 j}, e_{2 j^{\prime}} \text { independentes }
$$

ou seja,

$$
Y_{2 j} \mid x_{2} \sim N\left(\alpha+\beta x_{2} ; \sigma_{e e}\right) \quad, \quad j=1,2, \ldots, s_{2},
$$

de modo que

$$
\bar{Y}_{2}=\sum_{j=1}^{s_{2}} \frac{Y_{2 j}}{s_{2}} \sim N\left(\alpha+\beta x_{2} ; \frac{\sigma_{e e}}{s_{2}}\right) ; j=1,2, \ldots, s_{2} .
$$


Portanto, $\bar{Y}_{1}$ e $\bar{Y}_{2}$ são independentes

$$
\bar{Z}_{1}=\bar{Y}_{1}-\bar{Y}_{2} \sim N\left(\beta x_{1}-\beta x_{2} ; \frac{\sigma_{e e}}{s_{1}}+\frac{\sigma_{e e}}{s_{2}}\right) .
$$

Por outro lado, como

$$
\begin{aligned}
X_{1 k} & =x_{1}+u_{1 k}, u_{1 k} \sim N\left(0 ; \sigma_{u u}\right) \quad, k=1,2, \ldots, r_{1}, \\
X_{1 k} & \sim N\left(x_{1} ; \sigma_{u u}\right), \quad \text { são independentes, tem-se que } \\
\bar{X}_{1}=\sum_{k=1}^{r_{1}} \frac{X_{2 k}}{r_{1}} & \sim N\left(x_{1} ; \frac{\sigma_{u u}}{r_{1}}\right) . \\
X_{2 k} & =x_{2}+u_{2 k}, u_{2 k} \sim N\left(0 ; \sigma_{u u}\right) \quad ; \quad k=1,2, \ldots, r_{2}, \\
X_{2 k} & \sim N\left(x_{2} ; \sigma_{u u}\right) \quad \text { são independentes, têm-se que } \\
\bar{X}_{2}=\sum_{k=1}^{r_{2}} \frac{X_{2 k}}{r_{2}} & \sim N\left(x_{2} ; \frac{\sigma_{u u}}{r_{2}}\right),
\end{aligned}
$$

de onde segue que

$$
\begin{gathered}
\bar{Z}_{2}=\bar{X}_{1}-\bar{X}_{2} \sim N\left(x_{1}-x_{2} ; \frac{\sigma_{u u}}{r_{1}}+\frac{\sigma_{u u}}{r_{2}}\right) . \\
\therefore V(\widehat{\beta})=V\left(\frac{\bar{Z}_{1}}{\bar{Z}_{2}}\right)=V\left(\frac{\bar{Y}_{1}-\bar{Y}_{2}}{\bar{X}_{1}-\bar{X}_{2}}\right)
\end{gathered}
$$

Unindo (2.18 e 2.19) obtêm-se:

$$
\left[\begin{array}{l}
\bar{Y}_{1}-\bar{Y}_{2}-\beta\left(x_{1}-x_{2}\right) \\
\bar{X}_{1}-\bar{X}_{2}-\left(x_{1}-x_{2}\right)
\end{array}\right] \sim N\left(\left(\begin{array}{l}
0 \\
0
\end{array}\right) ;\left(\begin{array}{ll}
\frac{\sigma_{e e}}{s_{1}}+\frac{\sigma_{u u}}{s_{2}} & 0 \\
0 & \frac{\sigma_{e e}}{r_{1}}+\frac{\sigma_{u u}}{r_{2}}
\end{array}\right)\right)
$$

No Apêndice B, utilizando o Teorema B.1, garante que se $\sqrt{n}\left(T_{n}-\mu\right) \stackrel{D}{\rightarrow} N(0, \Sigma)$ e sendo $g$ uma função contínua, tem-se que $\sqrt{n}\left(g\left(T_{n}\right)-g(\mu)\right) \stackrel{D}{\rightarrow} N\left(0, g^{\bullet}\left(T_{n}\right) \Sigma g^{\bullet}\left(T_{n}\right)^{t}\right)$. 
Para utilizar este método é necessario fazer: $s_{1}=\frac{k}{k_{1}}, s_{2}=\frac{k}{k_{2}}, r_{1}=\frac{k}{k_{3}}$ e $r_{2}=\frac{k}{k_{4}}$, onde $\left\{k, k_{1}, k_{2}, k_{3}\right.$ e $\left.k_{4}\right\} \in \mathbf{N}$, de modo que a equação (2.21) seja expressa como:

$\sqrt{k}\left[\begin{array}{l}\bar{Y}_{1}-\bar{Y}_{2}-\beta\left(x_{1}-x_{2}\right) \\ \bar{X}_{1}-\bar{X}_{2}-\left(x_{1}-x_{2}\right)\end{array}\right] \sim N\left(\left(\begin{array}{l}0 \\ 0\end{array}\right) ;\left(\begin{array}{ll}k_{1} \sigma_{e e}^{2}+k_{2} \sigma_{u u}^{2} & 0 \\ 0 & k_{3} \sigma_{e e}^{2}+k_{4} \sigma_{u u}^{2}\end{array}\right)\right)$.

Usando

$$
\sqrt{k}\left(g\left(T_{n}\right)-g(\mu)\right) \stackrel{A}{\sim} N\left(0 ; g^{\bullet}(\mu) \Sigma g^{\bullet}(\mu)^{t}\right)
$$

têm-se que

$$
T_{n}=\left\{T_{n 1}, T_{n 2}\right\} \quad \text { e } \quad \mu=\left\{\mu_{1}, \mu_{2}\right\}
$$

obtendo

$$
\sqrt{k}\left(\frac{\bar{Y}_{1}-\bar{Y}_{2}}{\bar{X}_{1}-\bar{X}_{2}}-\beta \frac{\left(x_{1}-x_{2}\right)}{\left(x_{1}-x_{2}\right)}\right) \stackrel{A}{\sim} N\left(0 ; g^{\bullet}(\mu) \Sigma g^{\bullet}(\mu)^{t}\right)
$$

com

$$
g^{\bullet}(\mu)=\left(g_{1}^{\bullet}(\mu) ; g_{2}^{\bullet}(\mu)\right) \quad \text { e } g^{\bullet}(Z)=\left(g_{1}^{\bullet}\left(Z_{1}\right) ; g_{2}^{\bullet}\left(Z_{2}\right)\right)=\frac{Z_{1}}{Z_{2}}
$$

Portanto,

$$
\begin{gathered}
g_{1}^{\bullet}(Z)=\frac{\partial g\left(Z_{1} ; Z_{2}\right)}{\partial Z_{1}}=\frac{1}{Z_{2}} \quad \text { então, } \quad g_{1}^{\bullet}(\mu)=\frac{1}{E\left[Z_{2}\right]}=\frac{1}{x_{1}-x_{2}} \\
\text { e } g_{2}^{\bullet}(Z)=\frac{\partial g\left(Z_{1} ; Z_{2}\right)}{\partial Z_{2}}=\frac{-Z_{1}}{Z_{2}^{2}} \quad \text { então, } \quad g_{2}^{\bullet}(\mu)=\frac{E\left[Z_{1}\right]}{\left(E\left[Z_{2}\right]\right)^{2}}=\frac{-\beta\left(x_{1}-x_{2}\right)}{\left(x_{1}-x_{2}\right)^{2}}=\frac{-\beta}{x_{1}-x_{2}}
\end{gathered}
$$

ou seja, $g^{\bullet}(\mu)=\left(\frac{1}{x_{1}-x_{2}} ; \frac{-\beta}{x_{1}-x_{2}}\right)$,

de modo que,

$$
g^{\bullet}(\mu) \Sigma g^{\bullet}(\mu)^{t}=\left(\frac{1}{x_{1}-x_{2}}\right)^{2}\left(k_{1} \sigma_{e e}+k_{2} \sigma_{u u}\right)+\left(\frac{-\beta}{x_{1}-x_{2}}\right)^{2}\left(k_{3} \sigma_{e e}+k_{4} \sigma_{u u}\right),
$$

leva a $r_{1}, r_{2} \rightarrow \infty$ e $\bar{X}_{1} \stackrel{P}{\rightarrow} x_{1}, \bar{X}_{2} \stackrel{P}{\rightarrow} x_{2}$

$$
\sqrt{k}\left(\frac{\bar{Y}_{1}-\bar{Y}_{2}}{\bar{X}_{1}-\bar{X}_{2}}-\beta\right) \stackrel{A}{\sim} N\left(0 ; \frac{k_{1} \sigma_{e e}+k_{2} \sigma_{u u}}{\left(x_{1}-x_{2}\right)^{2}}+\frac{(-\beta)^{2}\left(k_{3} \sigma_{e e}+k_{4} \sigma_{u u}\right)}{\left(x_{1}-x_{2}\right)^{2}}\right) .
$$


O método delta mostra que com estimador de $\beta$ é possível encontrar a variança assintótica. Usando o Teorema B.1 do Apêndice B em (2.23) mostra-se que:

$$
E[\widehat{\beta}]=E\left[\frac{\bar{Y}_{1}-\bar{Y}_{2}}{\bar{X}_{1}-\bar{X}_{2}}\right] \cong \beta
$$

$\mathrm{e}$

$$
\begin{aligned}
V(\widehat{\beta}) & =V\left(\frac{\bar{Y}_{1}-\bar{Y}_{2}}{\bar{X}_{1}-\bar{X}_{2}}\right) \\
& \cong \frac{1}{k}\left\{\frac{k_{1} \sigma_{e e}+k_{2} \sigma_{u u}}{\left(x_{1}-x_{2}\right)^{2}}+\frac{(-\beta)^{2}\left(k_{3} \sigma_{e e}+k_{4} \sigma_{u u}\right)}{\left(x_{1}-x_{2}\right)^{2}}\right\} .
\end{aligned}
$$

Desenvolvendo algebraicamente $(2.25)$ encontra-se $V(\widehat{\beta})$, que é dada por:

$$
V(\widehat{\beta}) \cong \frac{1}{\left(x_{1}-x_{2}\right)^{2}}\left(\frac{\sigma_{e e}}{s_{1}}+\frac{\sigma_{u u}}{s_{2}}\right)+\left(\frac{\beta}{x_{1}-x_{2}}\right)^{2}\left(\frac{\sigma_{e e}}{r_{1}}+\frac{\sigma_{u u}}{r_{2}}\right)
$$




\subsection{Inferência usando verossimilhança perfilada}

Nesta seção mostra-se alguns resultados obtidos em Hokama (2001) e Dorf \& Gurland (1960), onde foram encontradas as propriedades dos estimadores de MV.

\subsubsection{Notações e resultados preliminares}

Sejam $Z_{1}, \ldots, Z_{n}$, uma sequência de vetores aleatórios $p$-dimensionais com função de log-verossimilhança dada por

$$
\sum_{i=1}^{n} \log f_{i}\left(\mathbf{z}_{i} ; \boldsymbol{\theta}, x_{i}\right),
$$

em que $f_{i}\left(\mathbf{z}_{i} ; \boldsymbol{\theta}, x_{i}\right)$ é a função densidade de $\mathbf{Z}_{i}, i=1, \ldots, n, \boldsymbol{\theta}=\left(\theta_{1}, \ldots, \theta_{p}\right)^{\prime} \in$ $\Theta \subset \mathcal{R}^{p}$ e $x_{i} \in \mathcal{X}_{i} \subset \mathcal{R}, i=1, \ldots, n$, são os parâmetros incidentais. Supomos que $\boldsymbol{\theta}^{0} \in \boldsymbol{\Theta}$ e $x_{i}^{0} \in \mathcal{X}_{i}, i=1, \ldots, n$, onde $\boldsymbol{\theta}^{0}$ e $x_{1}^{0}, \ldots, x_{n}^{0}$, denotam os verdadeiros valores dos parâmetros. Tomam-se valores esperados e as variâncias em relação a $\boldsymbol{\theta}^{0}$ e $x_{i}^{0}$, $i=1, \ldots, n$, e são denotados por $E_{0}[]=.E\left[\cdot \mid \boldsymbol{\theta}^{0}, x_{1}^{0}, \ldots, x_{n}^{0}\right]$. Para cada $i$ e dado $\theta$, seja $\tilde{x}_{i}=\tilde{x}_{i}\left(Z_{i}, \boldsymbol{\theta}\right)$ um estimador (dependente de $\boldsymbol{\theta}$ ) de $x_{i}$, com possibilidade de ser um estimador de máxima verossimilhança, obtido ao maximizar-se (2.27) em relação $x_{i}$ para $\boldsymbol{\theta}$ fixo.

Agora, substituindo $x_{i}$ por $\tilde{x}_{i}$ em (2.27), obtém-se

$$
\sum_{i=1}^{n} \log f_{i}\left(\mathbf{z}_{i} ; \boldsymbol{\theta}, \tilde{x}_{i}\right)=\sum_{i=1}^{n} h_{i}\left(\mathbf{z}_{i} ; \boldsymbol{\theta}\right) .
$$

Define-se as seguintes funções:

$$
\begin{gathered}
q_{i \theta_{j}}\left(\mathbf{z}_{i} ; \boldsymbol{\theta}\right)=\frac{\partial h_{i}\left(\mathbf{z}_{i} ; \boldsymbol{\theta}\right)}{\partial \theta_{j}}, \quad j=1, \ldots, p, \\
q_{i \theta_{k} \theta_{j}}\left(\mathbf{z}_{i} ; \boldsymbol{\theta}\right)=\frac{\partial^{2} h_{i}\left(\mathbf{z}_{i} ; \boldsymbol{\theta}\right)}{\partial \theta_{j} \partial \theta_{k}}, \quad j, k=1, \ldots, p,
\end{gathered}
$$


e

$$
q_{i \theta_{k}, \theta_{j}}\left(\mathbf{z}_{i} ; \boldsymbol{\theta}\right)=q_{i \theta_{k}}\left(\mathbf{z}_{i} ; \boldsymbol{\theta}\right) q_{i \theta_{j}}\left(\mathbf{z}_{i} ; \boldsymbol{\theta}\right), \quad j, k=1, \ldots, p .
$$

Além de disso, define-se $E_{0}\left[\mathbf{A}_{n}(\boldsymbol{\theta})\right]$ como a matriz $p \times p$, simétrica, com elemento $(j, k)$ dado por

$$
n^{-1} \sum_{i=1}^{n} E_{0}\left[q_{i \theta_{k} \theta_{j}}\left(\mathbf{Z}_{i} ; \boldsymbol{\theta}\right)\right], \quad j, k=1, \ldots, p
$$

Em Mak (1982), na Seção 2, considerações são establecidas sobre (2.28), de tal forma que o máximo $\tilde{\boldsymbol{\theta}}_{n}=\tilde{\boldsymbol{\theta}}\left(\mathbf{z}_{1}, \ldots, \mathbf{z}_{n}\right)$ converge em probabilidade para algum $\boldsymbol{\theta}^{1}$ no interior de $\boldsymbol{\Theta}$, onde $\boldsymbol{\theta}^{1}$ um máximo local da função

$$
\bar{\psi}(\boldsymbol{\theta})=n^{-1} \sum_{i=1}^{n} E_{0}\left[h_{i}\left(\mathbf{Z}_{i} ; \boldsymbol{\theta}\right)\right]
$$

e

$$
\sqrt{n}\left(\mathbf{V}_{n}\left(\boldsymbol{\theta}^{1}\right)\right)^{-1 / 2}\left(E_{0}\left[\mathbf{A}_{n}\left(\boldsymbol{\theta}^{1}\right)\right]\right)\left(\tilde{\boldsymbol{\theta}}_{n}-\boldsymbol{\theta}^{1}\right) \stackrel{A}{\sim} N_{p}\left(\mathbf{0}, \mathbf{I}_{p}\right)
$$

onde "A " significa convergência em distribuição, com o elemento $(j, k)$ das matrizes $p \times p, \mathbf{A}_{n}\left(\boldsymbol{\theta}^{1}\right)=\left(a_{j k}\right)$ e $\mathbf{V}_{n}\left(\boldsymbol{\theta}^{1}\right)=\left(v_{j k}\right)$, dados, respectivamente, por

$$
a_{j k}=\frac{1}{2} \sum_{i=1}^{n} q_{i \theta_{j} \theta_{k}}\left(\mathbf{Z}_{i} ; \boldsymbol{\theta}^{1}\right) \quad \text { e } \quad v_{j k}=\frac{1}{n} \sum_{i=1}^{n} \operatorname{Cov}\left[q_{i \theta_{j}}\left(\mathbf{Z}_{i}, \boldsymbol{\theta}^{1}\right), q_{i \theta_{k}}\left(\mathbf{Z}_{i}, \boldsymbol{\theta}^{1}\right)\right],
$$

donde, os valores esperados são realizados em relação aos verdadeiros valores $\boldsymbol{\theta}^{0} \mathrm{e}$ $x_{i}^{0}, i=1, \ldots, n$. Demostrações detalhadas são encontradas em Mak (1982) e Gimenez \& Bolfarine (1997). Mak (1982) mostra, também, que em algumas situações é possível obter estimadores $\tilde{x}_{i}$ de tal forma que $\boldsymbol{\theta}^{1}$ só depende de $\boldsymbol{\theta}^{0}$ (independente de $x_{i}^{0}$ ), isto é, existe uma função $g($.$) tal que \boldsymbol{\theta}^{1}=g\left(\boldsymbol{\theta}^{0}\right)$. Se $g$ é função um a um, um estimador consistente de $\boldsymbol{\theta}^{0}$, baseado em $\tilde{\boldsymbol{\theta}}_{n}$ pode ser construído considerando-se $\hat{\boldsymbol{\theta}}_{n}=g^{-1}\left(\tilde{\boldsymbol{\theta}}_{n}\right)$. 


\subsubsection{Comportamento assintótico dos EMV}

Nesta seção considera-se os resultados obtidos por Hokama (2001), que mostra um estimador de máxima verossimilhança de $\boldsymbol{\theta}^{\mathbf{0}}$ em relação a uma função $g^{-1}($.$) converte$ o estimador de MV de $\boldsymbol{\theta}^{\mathbf{0}}$ em um estimador consistente, $\widehat{\boldsymbol{\theta}}_{c}=g^{-1}\left(\widehat{\boldsymbol{\theta}}_{M V}\right) \stackrel{P}{\rightarrow} \boldsymbol{\theta}^{0}$.

Especificamente, mostrou-se que $\hat{\boldsymbol{\theta}}_{M V} \stackrel{P}{\rightarrow} \boldsymbol{\theta}^{1}=g\left(\boldsymbol{\theta}^{0}\right)$ onde a função $g($.$) apre-$ senta uma forma simples e a inversa $g^{-1}($.$) pode ser obtida levando a um estimador$ consistente $\hat{\boldsymbol{\theta}}_{c}$, em que $\hat{\boldsymbol{\theta}}_{c}=g^{-1}\left(\hat{\boldsymbol{\theta}}_{M V}\right)$.

Teorema 2.1 Dado $\boldsymbol{\theta}^{0}=\left(\alpha^{0}, \beta^{0}, \sigma_{e e}^{0}, \sigma_{u u}^{0}\right)$, os verdadeiros valores dos parâmetros estruturais, o EMV de $\hat{\boldsymbol{\theta}}$ converge em probabilidade para

$$
\boldsymbol{\theta}^{1}=\left(\alpha^{0}, \beta^{0}, \frac{(\bar{r}+\bar{s}-1)}{\bar{r}+\bar{s}} \sigma_{e e}^{0}, \frac{(\bar{r}+\bar{s}-1)}{\bar{r}+\bar{s}} \sigma_{u u}^{0}\right)^{\prime},
$$

no qual $\boldsymbol{\theta}^{1} \in \Theta^{\mathbf{0}}$ é o máximo local da função $\psi(\boldsymbol{\theta})=n^{-1} \sum_{i=1}^{n} E^{0}\left[h_{i}\left(\mathbf{Z}_{i} ; \boldsymbol{\theta}\right)\right]$, para $n$ grande e finito $\bar{r}=\lim _{n \rightarrow \infty} \sum_{k=1}^{n} r_{k} / n$ e $\bar{s}=\lim _{n \rightarrow \infty} \sum_{j=1}^{n} s_{j} / n$ e que dada a sequência $\left\{x_{i}\right\}$ onde

$$
\begin{gathered}
0<\lim _{n \rightarrow \infty} \inf \sum_{t=1}^{n} \frac{\left(x_{t}^{(o)}-\bar{x}^{(o)}\right)^{2}}{n} \leq \lim _{n \rightarrow \infty} \sup \frac{1}{n} \sum_{t=1}^{n}\left(x_{t}^{(o)}-\bar{x}^{(o)}\right)^{2}<\infty, \mathrm{e} \\
\lim _{n \rightarrow \infty} \frac{1}{n^{1+\frac{\gamma}{2}}} \sum_{t=1}^{n}\left|x_{t}\right|^{2+\gamma}=0,
\end{gathered}
$$

A prova do Teorema 2.1, encontra-se em Hokama (2001). Nota-se que o EMV de $\theta$, é assintoticamente normal com média $\boldsymbol{\theta}^{1}=\left(\alpha^{0}, \beta^{0}, \frac{(\bar{r}+\bar{s}-1)}{\bar{r}+\bar{s}} \sigma_{e e}^{0} \frac{(\bar{r}+\bar{s}-1)}{\bar{r}+\bar{s}} \sigma_{u u}^{0}\right)^{\prime}$, e a matriz de covariância é dada por $n^{-1}\left\{E^{0}\left[A_{n}\left(\theta^{(1)}\right)\right]\right\}^{-1} \mathbf{V}_{n}\left\{E^{0}\left[A_{n}\left(\theta^{(1)}\right)\right]\right\}^{-1}$. Com

$$
E_{0}\left[\mathbf{A}_{n}\left(\boldsymbol{\theta}^{1}\right)\right]=
$$




$$
=\left(\frac{w}{n \sigma_{u u}^{0}}\right)\left[\begin{array}{cccc}
-\sum_{i=1}^{n} v_{i} & -\sum_{i=1}^{n} v_{i} x_{i}^{0} & 0 & 0 \\
-\sum_{i=1}^{n} v_{i} x_{i}^{0} & -\sum_{i=1}^{n} v_{i} x_{i}^{0^{2}} & \lambda^{0} w \beta^{0} \Sigma_{r s} & -\beta^{0} w \Sigma_{r s} \\
0 & -\lambda \beta^{0} w \Sigma_{r s} & \frac{w^{2}}{\sigma_{u u}^{0}}\left(n c-\beta^{04} \Sigma_{s s}\right) & \frac{-\beta^{0} w^{2}}{\sigma_{u u}} \Sigma_{r s} \\
0 & -\beta^{0} w \Sigma_{r s} & \frac{-w^{2} \beta^{02}}{\sigma_{u u}} \Sigma_{r s} & \frac{w^{2}}{\lambda^{2} \sigma_{u u}^{0}}\left(n b-\lambda^{2} \Sigma_{r r}\right)
\end{array}\right]
$$

onde

$$
\begin{aligned}
& v_{i}=\frac{r_{i} s_{i}}{\Delta_{i}} ; \quad \Delta_{i}=r_{i} \lambda+s_{i} \beta^{0^{2}} ; \quad \Sigma_{r s}=\sum_{i=1}^{n} \frac{r_{i} s_{i}}{\Delta_{i}^{2}} ; \quad \Sigma_{r r}=\sum_{i=1}^{n} \frac{r_{i}^{2}}{\Delta_{i}^{2}} ; \quad \Sigma_{s s}=\sum_{i=1}^{n} \frac{s_{i}^{2}}{\Delta_{i}^{2}} \\
& w=\frac{\bar{r}+\bar{s}}{\bar{r}+\bar{s}-1} ; \quad \lambda=\frac{\sigma_{e e}}{\sigma_{u u}}=\frac{\sigma_{e e}^{0}}{\sigma_{u u}^{0}} .
\end{aligned}
$$

Agora, considerando as segundas derivadas e o valor esperado obteve-se:

$$
\begin{gathered}
\mathbf{V}_{n}\left(\boldsymbol{\theta}^{1}\right)=\left(\frac{1}{n \sigma_{u u}^{0}}\right) \\
\times\left[\begin{array}{cccc}
w^{2} \sum_{i=1}^{n} v_{i} & w^{2} \sum_{i=1}^{n} v_{i} x_{i}^{0} & 0 & 0 \\
w^{2} \sum_{i=1}^{n} v_{i} x_{i}^{0} & w^{2} \sum_{i=1}^{n} v_{i}\left(x_{i}^{02}+\frac{\lambda^{0} \sigma_{u u}^{0}}{\Delta_{i}}\right) & 0 & \\
0 & 0 & \frac{w^{4}}{2 \sigma_{u u}^{0}}\left(n(\bar{r}-1)+\beta^{04} \Sigma_{s s}\right) & \frac{\beta^{02} w^{4}}{2 \sigma_{u u}} \Sigma_{r s} \\
0 & 0 & \frac{\beta^{0} w^{4}}{2 \sigma_{u u}} \Sigma_{r s} & \frac{w^{4}}{2 \lambda^{2} \sigma_{u u}^{0}}\left(n(\bar{s}-1)+\lambda^{2} \Sigma_{r r}\right)
\end{array}\right],
\end{gathered}
$$

de modo que é possivel calcular a matriz de covariâncias assintóticas

$$
n^{-1}\left\{E^{0}\left[\mathbf{A}_{n}\left(\boldsymbol{\theta}^{1}\right)\right]\right\}^{-1} \mathbf{V}_{n}\left(\boldsymbol{\theta}^{1}\right)\left\{E^{0}\left[\mathbf{A}_{n}\left(\boldsymbol{\theta}^{1}\right)\right]\right\}^{-1}
$$

Aqui é dado o resultado assintótico da variância de $\widehat{\beta}_{M V}$, denotado por $\operatorname{AVAR}\left(\widehat{\beta}_{M V}\right)$,

$$
\operatorname{AVAR}\left(\hat{\beta}_{M V}\right)=\left[\frac{\lambda \sigma_{u u}^{o}}{D}\right]^{2}\left\{F+B C^{2}\right\}
$$


onde:

$$
\begin{aligned}
B & =\frac{1}{\lambda \sigma_{u u}^{o}}\left(\sum_{i=1}^{n} v_{i}\right)^{2}\left[\sum_{i=1}^{n} v_{i}\left(x_{i}^{0}-\bar{x}_{v}^{0}\right)^{2}+\sum_{i=1}^{n} \frac{v_{i} \lambda \sigma_{u u}^{0}}{\Delta_{i}}\right], \\
C & =\left\{\left[n\left(\bar{r}-\frac{\bar{r}}{2 w}\right)-\sum_{i=1}^{n} \frac{r_{i} \lambda}{\Delta_{i}}\right]\left[n\left(\bar{s}-\frac{\bar{s}}{2 w}\right)-\sum_{i=1}^{n} \frac{s_{i} \beta^{0^{2}}}{\Delta_{i}}\right]-\frac{n(\bar{r}+\bar{s})}{2 w} \sum_{i=1}^{n} \frac{v_{i} \lambda \beta^{0^{2}}}{\Delta_{i}}\right\}, \\
D & =\left\{\sum_{i=1}^{n} v_{i}\left(x_{i}^{0}-\bar{x}_{v}^{0}\right)^{2}\left[n\left(\bar{r}-\frac{\bar{r}}{2 w}\right)-\sum_{i=1}^{n} \frac{r_{i} \lambda}{\Delta_{i}}\right]\left[n\left(\bar{s}-\frac{\bar{s}}{2 w}\right)-\sum_{i=1}^{n} \frac{s_{i} \beta^{0^{2}}}{\Delta_{i}}\right]\right. \\
& \left.-\left[\frac{n(\bar{r}+\bar{s}-1)}{2} \sum_{i=1}^{n} \frac{v_{i} \lambda \beta^{0^{2}}}{\Delta_{i}}\right]\left[\sum_{i=1}^{n} v_{i}\left(x_{i}^{0}-\bar{x}_{v}^{0}\right)^{2}+\sum_{i=1}^{n} \frac{v_{i} \lambda \sigma_{u u}^{0}}{\Delta_{i}}\right]\right\}, \\
F & =\frac{n^{2}(\bar{r}+\bar{s})}{8 w^{2}}\left(\sum_{i=1}^{n} \frac{v_{i} \beta^{0}}{\Delta_{i}}\right)^{2}\left\{n \bar{s}(\bar{r}-1)-\frac{n \bar{r}^{2}}{\bar{r}+\bar{s}}-\sum_{i=1}^{n} \frac{\bar{r} r_{i}^{2} \lambda^{2}+\bar{s} s_{i}^{2} \beta^{0^{4}}}{\Delta_{i}^{2}}\right\} .
\end{aligned}
$$

Um estimador consistente da matriz de covariância pode ser implementado desenvolvendo o estimador "Sandwiche" de Gimenez \& Bolfarine (1997), com

$$
\mathbf{q}_{i}(\boldsymbol{\theta})=\frac{\partial h_{i}(\boldsymbol{\theta})}{\partial \boldsymbol{\theta}} \quad \text { e } \quad \mathbf{I}_{i}(\boldsymbol{\theta})=\frac{\partial^{2} h_{i}(\boldsymbol{\theta})}{\partial \boldsymbol{\theta} \partial \boldsymbol{\theta}^{\prime}}
$$

e considerando um estimador consistente assintoticamente da matrix de covariância (2.40), este é dado por

$$
\left(\overline{\mathbf{I}}_{n}\left(\hat{\boldsymbol{\theta}}_{c}\right)\right)^{-1} \overline{\mathbf{s}}_{n}\left(\hat{\boldsymbol{\theta}}_{c}\right)\left(\overline{\mathbf{I}}_{n}\left(\hat{\boldsymbol{\theta}}_{c}\right)\right)^{-1},
$$

onde

$$
\overline{\mathbf{s}}_{n}=\frac{1}{n} \sum_{i=1}^{n} \mathbf{q}_{i}(\boldsymbol{\theta}) \mathbf{q}_{i}(\boldsymbol{\theta})^{\prime}, \quad \text { e } \quad \overline{\mathbf{I}}_{n}(\boldsymbol{\theta})=\frac{1}{n} \sum_{i=1}^{n} \mathbf{I}_{i}(\boldsymbol{\theta}),
$$

$\operatorname{com} \boldsymbol{\theta}$ substituído pelo estimador de máxima verossimilhança $\hat{\boldsymbol{\theta}}$. 


\subsubsection{Estimadores obtidos por Dorf e Gurland}

Utilizando os estimadores obtidos em análise de variância, Dorf \& Gurland (1960) consideram os seguintes estimadores:

$$
\hat{\beta}_{D G 1}=\frac{B_{X Y}}{B_{X X}-W_{X X}} \quad \text { e } \quad \hat{\beta}_{D G 2}=\frac{B_{Y Y}-W_{Y Y}}{B_{X Y}^{\prime}},
$$

onde

$$
\begin{gathered}
B_{X X}=\sum_{i=1}^{n} \frac{r_{i}\left(\bar{X}_{i}-\bar{X}\right)^{2}}{n-1}, \quad W_{X X}=(R-n)^{-1} \sum_{i=1}^{n} \sum_{j=1}^{r_{i}}\left(X_{i j}-\bar{X}_{i}\right)^{2}, \\
B_{Y Y}=\sum_{i=1}^{n} \frac{s_{i}\left(\bar{Y}_{i}-\bar{Y}\right)^{2}}{n-1}, W_{Y Y}=(s-n)^{-1} \sum_{i=1}^{n} \sum_{j=1}^{s_{i}}\left(Y_{i j}-\bar{Y}_{i}\right)^{2}, \\
B_{X Y}=\sum_{i=1}^{n} \frac{r_{i}\left(\bar{X}_{i}-\bar{X}\right)\left(\bar{Y}_{i}-\bar{Y}\right)}{n-1} \quad \text { e } B_{X Y}^{\prime}=\sum_{i=1}^{n} \frac{s_{i}\left(\bar{X}_{i}-\bar{X}\right)\left(\bar{Y}_{i}-\bar{Y}\right)}{n-1},
\end{gathered}
$$

com

$$
R=\sum_{i=1}^{n} r_{i}, s=\sum_{i=1}^{n} s_{i}, \bar{X}_{i}=\sum_{k=1}^{r_{i}} \frac{X_{i k}}{r_{i}}, \bar{X}=\sum_{i=1}^{n} \frac{r_{i} \bar{X}_{i}}{R}, \bar{Y}_{i}=\sum_{j=1}^{r_{i}} \frac{Y_{i j}}{s_{i}}, \bar{Y}=\sum_{i=1}^{n} \frac{s_{i} \bar{Y}_{i}}{s} .
$$

As variâncias assintóticas são dadas por Dorf \& Gurland (1960), como seguem

$$
\begin{aligned}
A V A R\left(\hat{\beta}_{D G 1}\right) & =\frac{\beta^{2} \sigma_{u u}}{\sum_{i=1}^{n} r_{i}\left(x_{i}-\bar{x}\right)^{2}}+\frac{\sigma_{e e} \sum_{i=1}^{n}\left(r_{i}^{2} / s_{i}\right)\left(x_{i}-\bar{x}\right)^{2}}{\left(\sum_{i=1}^{n} r_{i}\left(x_{i}-\bar{x}\right)^{2}\right)^{2}} \\
& +\frac{n-1}{n} \frac{\sigma_{u u} \sigma_{e e} \sum_{i=1}^{n}\left(r_{i} / s_{i}\right)}{\left(\sum_{i=1}^{n} r_{i}\left(x_{i}-\bar{x}\right)^{2}\right)^{2}} \\
& +\frac{\beta^{2}\left\{Q_{1}\left(r_{H}, R\right) \sigma_{u u}^{2}+Q_{2}\left(r_{H}, R\right)\left(3 \sigma_{u u}^{2}\right)\right\}(n-1)^{2}}{\left(\sum_{i=1}^{n} r_{i}\left(x_{i}-\bar{x}\right)^{2}\right)^{2}},
\end{aligned}
$$




$$
\begin{aligned}
A V A R\left(\hat{\beta}_{D G 2}\right) & =\frac{\beta^{2} \sigma_{u u} \sum_{i=1}^{n}\left(s_{i}^{2} / s r_{i}\right)\left(x_{i}-\bar{x}\right)^{2}}{\left(\sum_{i=1}^{n} s_{i}\left(x_{i}-\bar{x}\right)^{2}\right)^{2}}+\frac{\sigma_{e e}}{\sum_{i=1}^{n} s_{i}\left(x_{i}-\bar{x}\right)^{2}} \\
& +\frac{n-1}{n} \frac{\sigma_{u u} \sigma_{e e} \sum_{i=1}^{n}\left(s_{i} / r_{i}\right)}{\left(\sum_{i=1}^{n} s_{i}\left(x_{i}-\bar{x}\right)^{2}\right)^{2}} \\
& +\frac{\left\{Q_{1}\left(s_{H}, s\right) \sigma_{e e}^{2}+Q_{2}\left(s_{H}, s\right)\left(3 \sigma_{e e}^{2}\right)\right\}(n-1)^{2}}{\beta^{2}\left(\sum_{i=1}^{n} s_{i}\left(x_{i}-\bar{x}\right)^{2}\right)^{2}},
\end{aligned}
$$

onde

$$
\begin{gathered}
r_{H}^{-1}=n^{-1} \sum_{i=1}^{n} r_{i}^{-1}, \quad s_{H}^{-1}=n^{-1} \sum_{i=1}^{n} s_{i}^{-1}, \\
Q_{1}\left(r_{H}, R\right)=\frac{1}{(n-1)^{2}}\left(2 n-2-\frac{3 n}{r_{H}}+\frac{6 n}{R}-\frac{3}{R}\right)+\frac{1}{(R-n)^{2}}\left(4 n-R-\frac{3 n}{r_{H}}\right) \\
+\frac{6}{R-n}-\frac{6 n}{(R-n)(n-1)}\left(\frac{1}{r_{H}}-\frac{1}{R}\right), \\
Q_{2}\left(r_{H}, R\right)=\frac{1}{(n-1)^{2}}\left(\frac{n}{r_{H}}-\frac{2 n}{R}+\frac{3}{R}\right)+\frac{1}{(R-n)^{2}}\left(\frac{n}{r_{H}}+R-2 n\right) \\
\quad+\frac{2}{R-n}-\frac{2 n}{(R-n)(n-1)}\left(\frac{1}{r_{H}}-\frac{1}{R}\right),
\end{gathered}
$$

com suas correspondentes definições para $Q_{1}\left(s_{H}, s\right)$ e $Q_{2}\left(s_{H}, s\right)$. 


\subsubsection{Comparação dos estimadores de MV e Dorf e Gurland}

Nesta seção apresenta-se resultados de simulações realizadas para o cálculo do estimador de máxima verossimilhança e os estimadores propostos por Dorf \& Gurland (1960).

\section{Resultados}

Nas tabelas a seguir mostram-se que, mediante os dois métodos de estimação propostos para estimar $\alpha$ e $\beta$, os estimados são quase assintoticamente não viesados, sendo que Dorf \& Gurland (1960) só propõe estimadores para os parâmetros $\alpha$ e $\beta$. Observa-se através das Tabelas 2.5, 2.6, 2.7 e 2.8 que o melhor estimador para o parâmetro $\beta$ é o determinado pelo método de máxima verossimilhança pois o erro quadrático médio empírico (EQM E) $E Q M E\left(\widehat{\beta}_{M V}\right)<E Q M E\left(\widehat{\beta}_{\text {Dorf }}\right)$. Mas, quando $n$ aumenta os dois métodos são mais parecidos e não depende dos valores dos vetores $\boldsymbol{r}$ e $\boldsymbol{s}$. O estimador de $\alpha$ não é de interesse direto nestas análises pois este depende do estimador de $\beta$.

Recomenda-se então usar o estimador de máxima verossimilhança e não os estimadores propostos por Dorf e Gurland (1960). No próximo capítulo considera-se um procedimento alternativo o qual é comparado com os estimadores de máxima verossimilhança (EMV). 
Tabela 2.5: Vício e erro quadrático médio empírico usando 5000 amostras simuladas, com tamanho de amostra $n=3$, réplicas de $s=(8,10,15)$ e $r=(16,20,30)$.

\begin{tabular}{c|cc|cc}
\hline & \multicolumn{2}{|c|}{ M.V } & \multicolumn{2}{c}{ Dorf e Gurland } \\
\hline Parâmetro & Vício & EQM & Vício & EQM \\
\hline$\alpha$ & $-0,09168665$ & 568,9939 & 0,03315767 & 0,1119013 \\
$\beta$ & 0,007735509 & 5,842645 & $-6,242035$ & 163484,8 \\
$\sigma_{e e}$ & $-0,04515078$ & 0,0615871 & & \\
$\sigma_{u u}$ & $-0,01090724$ & 0,03144607 & & \\
\hline
\end{tabular}

Os valores verdadeiros utilizados na simulação foram: $\alpha=1, \beta=1, \sigma_{e e}=1, \sigma_{u u}=1$ e $x_{i} \sim N\left(\mu_{x} ; \sigma_{x x}\right)$, com $\mu_{x}=10$ e $\sigma_{x x}=1$.

Tabela 2.6: Vício e erro quadrático médio empírico usando 5000 amostras simuladas, com tamanho de amostra $n=3$, réplicas de $s=(30,40,60)$ e $r=(40,60,80)$.

\begin{tabular}{c|cc|cc}
\hline & \multicolumn{2}{|c|}{ M.V } & \multicolumn{2}{c}{ Dorf e Gurland } \\
\hline Parâmetro & Vício & EQM & Vício & EQM \\
\hline$\alpha$ & $-0,2262309$ & 31,76402 & 0,01252816 & 0,02846771 \\
$\beta$ & 0,02218437 & 0,2818339 & 0,02836179 & 2,172961 \\
$\sigma_{e e}$ & $-0,008478034$ & 0,01562143 & & \\
$\sigma_{u u}$ & $-0,004959637$ & 0,01154293 & & \\
\hline
\end{tabular}

Os valores verdadeiros utilizados na simulação foram: $\alpha=1, \beta=1, \sigma_{e e}=1, \sigma_{u u}=1$ e $x_{i} \sim N\left(\mu_{x} ; \sigma_{x x}\right), \operatorname{com} \mu_{x}=10$ e $\sigma_{x x}=3$. 
Tabela 2.7: Vício e erro quadrático médio empírico usando 5000 amostras simuladas, com tamanho de amostra $n=15$, réplicas variando entre $\mathrm{s}=(6,9,12$, $15,18,21,24,21,18,15,12,9,6,9,12)$ e $\mathrm{r}=(11,14,17,20,23,26,29,26,23,20,17,14,11,14,17)$.

\begin{tabular}{c|cc|cc}
\hline & \multicolumn{2}{|c|}{ M.V } & \multicolumn{2}{c}{ Dorf e Gurland } \\
\hline Parâmetro & Vício & EQM & Vício & EQM \\
\hline$\alpha$ & $-0,009302872$ & 0,3805245 & 0,007252162 & 0,01792240 \\
$\beta$ & 0,000813486 & 0,003706167 & 0,00227598 & 0,003819574 \\
$\sigma_{e e}$ & $-0,0054887$ & 0,01012255 & & \\
$\sigma_{u u}$ & $-0,003883411$ & 0,00753953 & & \\
\hline
\end{tabular}

Os valores verdadeiros utilizados na simulação foram: $\alpha=1, \beta=1, \sigma_{e e}=1, \sigma_{u u}=1$ e $x_{i} \sim N\left(\mu_{x} ; \sigma_{x x}\right)$, com $\mu_{x}=10$ e $\sigma_{x x}=3$.

Tabela 2.8: Vício e erro quadrático médio empírico usando 5000 amostras simuladas, com tamanho de amostra $n=30$, réplicas variando entre $\mathrm{s}=(6,9,12, \quad 15,18,21,24,21,18,15,12,9,6,9,12,15,18,21,24,21,18,15,12,9,6,9,12,15,18,21)$ e $\quad \mathrm{r}=(11,14,17,20,23,26,29,26,23,20,17,14,11,14,17,20,23,26,29,26,23,20,17$, $14,11,14,17,20,23,26)$.

\begin{tabular}{c|cc|cc}
\hline & \multicolumn{2}{|c|}{ M.V } & \multicolumn{2}{c}{ Dorf e Gurland } \\
\hline Parâmetro & Vício & EQM & Vício & EQM \\
\hline$\alpha$ & $-0,001254135$ & 0,1515084 & 0,003208802 & 0,008488439 \\
$\beta$ & 0,0001330337 & 0,001479113 & 0,0006121384 & 0,001510466 \\
$\sigma_{e e}$ & $-0,002464917$ & 0,004885954 & & \\
$\sigma_{u u}$ & $-0,002134302$ & 0,003528805 & & \\
\hline
\end{tabular}

Os valores verdadeiros utilizados na simulação foram: $\alpha=1, \beta=1, \sigma_{e e}=1, \sigma_{u u}=1$ e $x_{i} \sim N\left(\mu_{x} ; \sigma_{x x}\right)$, com $\mu_{x}=10$ e $\sigma_{x x}=3$. 


\section{Capítulo 3}

\section{O Enfoque do escore corrigido}

\subsection{Introdução}

O enfoque do escore corrigido permite a obtenção de estimadores consistentes para a situação funcional bem como para situações generalizadas. Baseando-se no fato de que a consistência dos estimadores pode ser obtida como solução de uma equação de estimação não viciada, Nakamura (1990) e Gimenez \& Bolfarine (1997) propõem o método do escore para estimação e testes de hipóteses em modelos com erro nas variáveis, que pode ser aplicado a modelos funcionais e estruturais. A partir de uma correção do logaritmo da função de verossimilhança "naive" (ingênua), obtida substituindo $x_{i}$ por $\bar{X}_{i}$, Nakamura (1990) define uma função escore dependendo das covariáveis observadas, cuja esperança em relação à distribuição dos erros de medida, coincide com a função escore usual baseada nas verdadeiras covariáveis desconhecidas. A seguir descrevemos o método que leva a estimadores consistentes e assintoticamente normais. 


\subsection{Descrição do método}

Seja $\theta=\left(\theta_{\mathbf{1}}, \ldots, \theta_{\mathbf{p}}\right)$ o vetor $p \times 1$ de parâmetros desconhecidos que pertence ao espaço paramétrico $\Theta$. Denota-se por $l(\theta \mid \mathbf{x}, \mathbf{Y})$, o logaritmo da função de verossimilhança, $U(\theta \mid \mathbf{x}, \mathbf{Y})$, a função escore e $I(\theta \mid \mathbf{x}, \mathbf{Y})=-\partial \mathbf{U}(\theta \mid \mathbf{x}, \mathbf{Y}) / \partial \theta$, a matriz de informação observada, de $\mathbf{x}$ e $\mathbf{Y}$, onde $\theta \in \boldsymbol{\Theta}$, um subconjunto convexo aberto de $\boldsymbol{\Theta}$. Sejam $\theta_{0}$ o verdadeiro valor do parâmetro $\left(\theta_{\mathbf{0}} \in \boldsymbol{\Theta}\right)$ e $E^{+}$a esperança com relação ao vetor aleatório $Y$ então,

$$
E^{+}\left\{U\left(\theta_{0} \mid \mathbf{x}, \mathbf{Y}\right)\right\}=0
$$

Quando $\mathbf{x}$ é sujeita a erro e $\mathbf{X}$ é a quantidade observada de $\mathbf{x}$, chamamos de $U(\theta \mid \mathbf{X}, \mathbf{Y})$ a função escore ingênua, onde $\theta \in \boldsymbol{\Theta}$. Então, em geral, $E^{+} U\left(\theta_{\mathbf{0}} \mid \mathbf{X}, \mathbf{Y}\right) \neq$ 0 , implicando que a solução $\widehat{\theta}_{\mathbf{w}}$ de $\left.U\left(\widehat{\theta}_{\mathbf{w}} \mid \mathbf{X}, \mathbf{Y}\right)\right)=\mathbf{0}$, não é necessariamente um estimador consistente de $\theta$. Para corrigir este problema, Nakamura (1990) propõe a log-verossimilhança corrigida $l^{*}(\theta \mid \mathbf{X}, \mathbf{Y})$, que satisfaz a condição

$$
E^{*}\left(l^{*}(\theta \mid \mathbf{X}, \mathbf{Y})\right)=\mathbf{l}(\theta \mid \mathbf{x}, \mathbf{Y}),
$$

onde $E^{*}$ denota a média condicional com relação a $\mathbf{X}$ dado $\mathbf{x}, \mathbf{Y}$ e $\theta \in \boldsymbol{\Theta}$. Sendo $\left.U^{*}(\theta \mid \mathbf{X}, \mathbf{Y})\right)=\partial \mathbf{l}^{*}(\theta \mid \mathbf{X}, \mathbf{Y}) / \partial \theta$ e $I^{*}(\theta \mid \mathbf{X}, \mathbf{Y})=\partial \mathbf{U}^{*}(\theta \mid \mathbf{X}, \mathbf{Y}) / \partial \theta$ a função escore corrigida e a matriz de informação corrigida, respectivamente, e se $E^{*}$ e $\partial \theta$ são permutáveis; então

$$
E^{*} U^{*}(\theta \mid \mathbf{X}, \mathbf{Y})=U(\theta \mid \mathbf{x}, \mathbf{Y}) \quad \text { e } \quad \mathbf{E}^{*}\left(\mathbf{I}^{*}(\theta \mid \mathbf{X}, \mathbf{Y})\right)=\mathbf{I}(\theta \mid \mathbf{x}, \mathbf{Y})
$$

Finalmente, chamamos de estimador do escore corrigido de $\theta, \widehat{\theta}^{*}$ tal que $U^{*}\left(\widehat{\theta}^{*} \mid \mathbf{X}, \mathbf{Y}\right)=$ $\mathbf{0}$, com $I^{*}\left(\widehat{\theta}^{*} \mid \mathbf{X}, \mathbf{Y}\right)$ sendo positiva definida.

Segundo Nakamura (1990), a verossimilhança corrigida $l^{*}$ e o estimador corrigido têm boas propriedades. Se $E=E^{+} E^{*}$ denota a esperança global (incondicional), 
então utilizando (3.1) e (3.3) segue que

$$
E U^{*}\left(\theta_{\mathbf{0}} \mid \mathbf{X}, \mathbf{Y}\right)=E^{+} E^{*} U^{*}\left(\theta_{\mathbf{0}} \mid \mathbf{X}, \mathbf{Y}\right)=E^{+} U\left(\theta_{\mathbf{0}} \mid \mathbf{x}, \mathbf{Y}\right)=0
$$

Isto indica que a função escore corrigido é uma função escore não viciada (Stefanski e Carroll (1987), Nakamura (1990)). Em particular, como mostrado por Nakamura (1990), o estimador $\widehat{\theta}^{*}$ tem propriedades de consistência e normalidade assintótica, sob certas condições de regularidade.

Segundo Gimenez (1997), define a função escore corrigida da seguinte maneira:

Definição 3.1 Uma função $U^{*}(\theta ; \mathbf{X}, \mathbf{Y})$ é dita função escore corrigida se

$$
E\left[U^{*}(\theta ; \mathbf{X}, \mathbf{Y}) \mid \mathbf{x}, \mathbf{Y}\right]=U^{*}(\theta ; \mathbf{x}, \mathbf{Y}) \quad \forall \theta \in \mathbf{\Theta}
$$

Sobre certas condições de regularidade (Apêndice A), existe uma seqüência consistente e assintoticamente normal $\hat{\theta}_{n}$, de soluções da equação

$$
U^{*}(\theta ; \mathbf{X}, \mathbf{Y})=\mathbf{0}
$$

A seguir, mostra-se alguns resultados assintóticos relacionados com este método.

\subsubsection{Condições de regularidade}

Vários autores têm proposto diferentes condições de regularidade sob certas restrições. O enfoque usual de máxima verossimilhança no caso de observações independentes e identicamentes distribuídas produz estimadores consistentes e assintoticamente normais Norden (1973) e Hokama et al. (2001). A maioria dos trabalhos não estudam 
a estimativa de MV na presença de parâmetros incidentais. Mak (1982) propõe uma técnica de estimação enfocando o uso da máxima verossimilhança na presença de parâmetros incidentais e estabelece condições de regularidade. Condições de regularidade suficientes para a validade dos resultados assintóticos enfocando o método do escore corrigido estão apresentados em Gimenez (1997) e são apresentadas no Apêndice A.

\subsection{Aplicação do método com $\sigma_{u u}$ conhecido}

O logaritmo da função de verossimilhança "não observada" é dado por

$$
\ell(\theta, \mathbf{x}, \mathbf{Y})=\sum_{i=1}^{n} \ell_{i}(\theta, \mathbf{x}, \mathbf{Y})
$$

onde

$$
\ell_{i} \propto-\frac{1}{2}\left[s_{i} \log \left(\sigma_{e e}\right)\right]-\frac{1}{2}\left\{\sigma_{e e}^{-1} \sum_{j=1}^{s_{i}}\left(Y_{i j}-\alpha-\beta x_{i}\right)^{2}\right\}
$$

tal que

$$
\ell \propto-\frac{1}{2} \sum_{i=1}^{n}\left[s_{i} \log \left(\sigma_{e e}\right)\right]-\frac{1}{2}\left\{\sigma_{e e}^{-1} \sum_{i=1}^{n} \sum_{j=1}^{s_{i}}\left(Y_{i j}-\alpha-\beta x_{i}\right)^{2}\right\} .
$$

Denota-se por $\ell^{\bullet}$ a função log-verossimilhança ingênua que segue substituindo $x_{i}=$ $\bar{X}_{i}$, em (3.8),

$$
\ell \propto-\frac{1}{2} \sum_{i=1}^{n}\left[s_{i} \log \left(\sigma_{e e}\right)\right]-\frac{1}{2}\left\{\sigma_{e e}^{-1} \sum_{i=1}^{n} \sum_{j=1}^{s_{i}}\left(Y_{i j}-\alpha-\beta \bar{X}_{i}\right)^{2}\right\},
$$


ou seja,

$$
\ell^{\bullet}=\sum_{i=1}^{n} \ell_{i}^{\bullet}
$$

onde

$$
\ell_{i}^{\bullet} \propto-\frac{1}{2}\left[s_{i} \log \left(\sigma_{e e}\right)\right]-\frac{1}{2}\left\{\sigma_{e e}^{-1} \sum_{j=1}^{s_{i}}\left(Y_{i j}-\alpha-\beta \bar{X}_{i}\right)^{2}\right\}
$$

de modo que

$$
\begin{aligned}
E\left[\left(Y_{i j}-\alpha-\beta \bar{X}_{i}\right)^{2} \mid x, Y\right]= & \left.E\left[\left(Y_{i j}-\alpha-\beta x_{i}\right)-\beta\left(\bar{X}_{i}-x_{i}\right)\right)^{2} \mid x, Y\right] \\
= & E\left[\left(Y_{i j}-\alpha-\beta x_{i}\right)^{2}+\left(-\beta\left(\bar{X}_{i}-x_{i}\right)\right)^{2}\right. \\
& \left.-2\left(Y_{i j}-\alpha-\beta x_{i}\right)\left(\beta\left(\bar{X}_{i}-x_{i}\right)\right) \mid x, Y\right] \\
= & \left(Y_{i j}-\alpha-\beta x_{i}\right)^{2}+\frac{\beta^{2} \sigma_{u u}}{r_{i}},
\end{aligned}
$$

pode-se, utilizar este resultado na seguinte equação:

$$
\begin{aligned}
E\left[\ell_{i}^{\bullet} \mid x, Y\right] & =-\frac{1}{2}\left[s_{i} \log \left(\sigma_{e e}\right)\right]-\frac{1}{2}\left\{\sigma_{e e}^{-1} \sum_{j=1}^{s_{i}} E\left[\left(Y_{i j}-\alpha-\beta \bar{X}_{i}\right)^{2} \mid x, Y\right]\right\}+c t e \\
& =-\frac{1}{2}\left[s_{i} \log \left(\sigma_{e e}\right)\right]-\frac{1}{2}\left[\sigma_{e e}^{-1} \sum_{j=1}^{s_{i}}\left\{\left(Y_{i j}-\alpha-\beta x_{i}\right)^{2}+\frac{\beta^{2} \sigma_{u u}}{r_{i}}\right\}\right]+c t e \\
& =-\frac{1}{2}\left[s_{i} \log \left(\sigma_{e e}\right)\right]-\frac{1}{2}\left\{\sigma_{e e}^{-1} \sum_{j=1}^{s_{i}}\left(Y_{i j}-\alpha-\beta x_{i}\right)^{2}\right\}-\frac{\sigma_{e e}^{-1}}{2}\left\{\frac{\beta^{2} \sigma_{u u} s_{i}}{r_{i}}\right\}+c t e \\
E\left[\ell_{i}^{\bullet} \mid x, Y\right] & =\ell_{i}-\frac{1}{2}\left\{\sigma_{e e}^{-1} \frac{\beta^{2} \sigma_{u u} s_{i}}{r_{i}}\right\},
\end{aligned}
$$

dado que $A_{i}=-\frac{1}{2}\left\{\sigma_{e e}^{-1} \frac{\beta^{2} \sigma_{u u} s_{i}}{r_{i}}\right\}$ e $A=\sum_{i=1}^{n} A_{i}$. Pode-se notar que $E\left[\ell_{i}^{\bullet} \mid x, Y\right]=$ $\ell_{i}+A_{i}$, a função de log-verossimilhança corrigida é dada por $\ell_{i}^{*}=\ell_{i}^{\bullet}-A_{i}$ que é 
definida por:

$$
\ell^{*}=\sum_{i=1}^{n}\left(\ell_{i}^{\bullet}-A_{i}\right)
$$

ou seja,

$$
\ell^{*} \propto-\frac{1}{2} \sum_{i=1}^{n} s_{i} \log \left(\sigma_{e e}\right)-\frac{1}{2}\left\{\sigma_{e e}^{-1} \sum_{i=1}^{n} \sum_{j=1}^{s_{i}}\left(Y_{i j}-\alpha-\beta \bar{X}_{i}\right)^{2}\right\}+\frac{1}{2} \sigma_{e e}^{-1} \beta^{2} \sigma_{u u} \sum_{i=1}^{n} \frac{s_{i}}{r_{i}} .
$$

O resultado é obtido devido a única função que satisfaz $E\left[\ell^{*}(\theta, \mathbf{X}, \mathbf{Y}) \mid \mathbf{x}, \mathbf{Y}\right]=$ $\ell(\theta, \mathbf{x}, \mathbf{Y})$ e explica porque $\bar{X}_{i}$ e $\bar{X}_{i}^{2}-\sigma_{u u} / r_{i}$ são os únicos estimadores não viciados de $x_{i}$ e $x_{i}^{2}$, respectivamente.

Portanto, os estimadores do escore corrigido são obtidos calculando-se:

$$
\frac{\partial \ell_{i}^{*}}{\partial \alpha}=\sigma_{e e}^{-1} s_{i}\left\{\bar{Y}_{i}-\alpha-\beta \bar{X}_{i}\right\}
$$

que leva a equação

$$
\sigma_{e e}^{-1} \sum_{i=1}^{n} s_{i}\left\{\bar{Y}_{i}-\alpha-\beta \bar{X}_{i}\right\}=0
$$

ou seja,

$$
\widehat{\alpha}^{*}=\frac{\sum_{i=1}^{n} s_{i} \bar{Y}_{i}-\widehat{\beta}^{*} \sum_{i=1}^{n} s_{i} \bar{X}_{i}}{\sum_{i=1}^{n} s_{i}} .
$$

Com relação a $\beta$, temos

$$
\frac{\partial \ell_{i}^{*}}{\partial \beta}=s_{i} \bar{X}_{i} \sigma_{e e}^{-1}\left(\bar{Y}_{i}-\widehat{\alpha}^{*}-\widehat{\beta}^{*} \bar{X}_{i}\right)+\frac{\sigma_{u u} \widehat{\beta}^{*} s_{i}}{\sigma_{e e} r_{i}}
$$


e como

$$
\frac{\partial \ell^{*}}{\partial \beta}=\sum_{i=1}^{n} \frac{\partial \ell_{i}^{*}}{\partial \beta}=0
$$

temos a equação

$$
0=\sum_{i=1}^{n} s_{i} \bar{X}_{i} \sigma_{e e}^{-1}\left(\bar{Y}_{i}-\widehat{\alpha}^{*}-\widehat{\beta}^{*} \bar{X}_{i}\right)+\sum_{i=1}^{n} \frac{\sigma_{u u} \widehat{\beta}^{*} s_{i}}{\sigma_{e e} r_{i}},
$$

ou seja

$$
\sum_{i=1}^{n} s_{i} \bar{X}_{i} \sigma_{e e}^{-1} \bar{Y}_{i}-\alpha \sum_{i=1}^{n} s_{i} \bar{X}_{i} \sigma_{e e}^{-1}=\beta\left\{\sum_{i=1}^{n} s_{i} \bar{X}_{i}^{2} \sigma_{e e}^{-1}-\frac{\sigma_{u u}}{\sigma_{e e}} \sum_{i=1}^{n} \frac{s_{i}}{r_{i}}\right\}
$$

Substituindo $\widehat{\alpha}$ em (3.17), têm-se

$$
\begin{array}{r}
\sum_{i=1}^{n} s_{i} \bar{X}_{i} \sigma_{e e}^{-1} \bar{Y}_{i}-\left\{\frac{\sum_{i=1}^{n} s_{i} \bar{Y}_{i}-\widehat{\beta}^{*} \sum_{i=1}^{n} s_{i} \bar{X}_{i}}{\sum_{i=1}^{n} s_{i}}\right\}\left(\sum_{i=1}^{n} s_{i} \bar{X}_{i} \sigma_{e e}^{-1}\right)= \\
\widehat{\beta}^{*}\left\{\sum_{i=1}^{n} s_{i} \bar{X}_{i}^{2} \sigma_{e e}^{-1}-\frac{\sigma_{u u}}{\sigma_{e e}} \sum_{i=1}^{n} \frac{s_{i}}{r_{i}}\right\},
\end{array}
$$

ou seja

$$
\begin{array}{r}
\sum_{i=1}^{n} s_{i} \bar{X}_{i} \sigma_{e e}^{-1} \bar{Y}_{i}-\left(\frac{\sum_{i=1}^{n} s_{i} \bar{Y}_{i}}{\sum_{i=1}^{n} s_{i}}\right)\left(\sum_{i=1}^{n} s_{i} \bar{X}_{i} \sigma_{e e}^{-1}\right)= \\
\widehat{\beta}^{*}\left\{\sum_{i=1}^{n} s_{i} \bar{X}_{i}^{2} \sigma_{e e}^{-1}-\frac{\sigma_{u u}}{\sigma_{e e}} \sum_{i=1}^{n} \frac{s_{i}}{r_{i}}-\sum_{i=1}^{n} s_{i} \bar{X}_{i} \sigma_{e e}^{-1} \frac{\sum_{i=1}^{n} s_{i} \bar{X}_{i}}{\sum_{i=1}^{n} s_{i}}\right\},
\end{array}
$$

que fornece o estimador com forma analítica

$$
\widehat{\beta}^{*}=\frac{\sum_{i=1}^{n} s_{i} \bar{X}_{i} \bar{Y}_{i}-\left(\frac{\sum_{i=1}^{n} s_{i} \bar{Y}_{i}}{\sum_{i=1}^{n} s_{i}}\right)\left(\sum_{i=1}^{n} s_{i} \bar{X}_{i}\right)}{\sum_{i=1}^{n} s_{i} \bar{X}_{i}^{2}-\sigma_{u u} \sum_{i=1}^{n} \frac{s_{i}}{r_{i}}-\frac{\left(\sum_{i=1}^{n} s_{i} \bar{X}_{i}\right)^{2}}{\sum_{i=1}^{n} s_{i}}} .
$$

Portanto, $\widehat{\beta}^{*}$ não necessita de procedimento iterativo. Da mesma forma

$$
\frac{\partial \ell_{i}^{*}}{\partial \sigma_{e e}}=-\frac{s_{i}}{2 \sigma_{e e}}+\frac{1}{2 \sigma_{e e}^{2}} \sum_{j=1}^{s_{i}}\left(Y_{i j}-\alpha-\beta \bar{X}_{i}\right)^{2}-\frac{s_{i} \beta^{2} \sigma_{u u}}{2 \sigma_{e e}^{2} r_{i}},
$$


e como

$$
\begin{array}{r}
\frac{\partial \ell^{*}}{\partial \sigma_{e e}}=\sum_{i=1}^{n} \frac{\partial \ell_{i}^{*}}{\partial \sigma_{e e}}=0, \\
0=-\frac{1}{2 \sigma_{e e}} \sum_{i=1}^{n} s_{i}+\frac{1}{2 \sigma_{e e}^{2}} \sum_{i=1}^{n} \sum_{j=1}^{s_{i}}\left(Y_{i j}-\widehat{\alpha}^{*}-\widehat{\beta}^{*} \bar{X}_{i}\right)^{2}-\frac{\widehat{\beta}^{* 2} \sigma_{u u}}{2 \sigma_{e e}^{2}} \sum_{i=1}^{n} \frac{s_{i}}{r_{i}}
\end{array}
$$

que resulta no estimador

$$
\widehat{\sigma}_{e e}^{*}=\frac{\sum_{i=1}^{n} \sum_{j=1}^{s_{i}}\left(Y_{i j}-\widehat{\alpha}^{*}-\widehat{\beta}^{*} \bar{X}_{i}\right)^{2}-\widehat{\beta}^{* 2} \sigma_{u u} \sum_{i=1}^{n} \frac{s_{i}}{r_{i}}}{\sum_{i=1}^{n} s_{i}} .
$$

Um estimador não viesado para $\sigma_{u u}$ pode ser obtido a partir das repetições, sendo representado por

$$
\widehat{\sigma}_{u u}=\frac{\sum_{i=1}^{n} \sum_{k=1}^{r_{i}}\left(X_{i k}-\bar{X}_{i}\right)^{2}}{\sum_{k=1}^{n} r_{i}-n}
$$

\subsubsection{Cálculos para encontrar estimador para a variância as- sintótica}

A expressão da variância assintótica para o método do escore corrigido é dada por $\left\{\bar{\Lambda}_{n}\left(\theta_{0}\right)\right\}^{-1} \bar{\Gamma}_{n}\left(\theta_{0}\right)\left\{\bar{\Lambda}_{n}\left(\theta_{0}\right)^{\prime}\right\}^{-1}$, sendo $\left\{\bar{I}_{n}^{*}(\theta)\right\}^{-1} \bar{s}_{n}^{*}(\theta)\left\{\bar{I}_{n}^{*}(\theta)^{\prime}\right\}^{-1}$ o estimador consistente, onde $\bar{s}_{n}^{*}(\theta)=\frac{1}{n} \sum_{i=1}^{n} U_{i}^{*}(\theta) U_{i}^{*}(\theta)^{\prime}, \bar{I}_{n}^{*}(\theta)=\frac{1}{n} I^{*}(\theta)=\frac{1}{n} \sum_{i=1}^{n} I_{i}^{*}(\theta)$, ver Gimenez (1997).

Portanto, o estimador da variância assintótica é obtido calculando-se:

$$
U_{i \alpha}^{*}=\frac{\partial \ell_{i}^{*}}{\partial \alpha}=\sigma_{e e}^{-1}\left\{s_{i} \bar{Y}_{i}-s_{i} \alpha-\beta \bar{X}_{i} s_{i}\right\},
$$

e

$$
U_{i \alpha \alpha}^{*}=\frac{\partial^{2} \ell_{i}^{*}}{\partial^{2} \alpha}=-s_{i} \sigma_{e e}^{-1} .
$$


Desta forma tem-se a derivada de segunda ordem com relação a $\alpha$ e $\beta$

$$
U_{i \alpha \beta}^{*}=\frac{\partial^{2} \ell_{i}^{*}}{\partial \beta \partial \alpha}=-s_{i} \bar{X}_{i} \sigma_{e e}^{-1},
$$

como também para $\alpha$ e $\sigma_{e e}$

$$
U_{i \alpha \sigma_{e e}}^{*}=\frac{\partial^{2} \ell_{i}^{*}}{\partial \sigma_{e e} \partial \alpha}=-\sigma_{e e}^{-2} s_{i}\left\{\bar{Y}_{i}-\alpha-\beta \bar{X}_{i}\right\} .
$$

Da mesma forma é realizado para $\beta$

$$
U_{i \beta}^{*}=\frac{\partial \ell_{i}^{*}}{\partial \beta}=s_{i} \bar{X}_{i} \sigma_{e e}^{-1}\left\{\bar{Y}_{i}-\alpha-\beta \bar{X}_{i}\right\}+\frac{\sigma_{u u} \beta s_{i}}{\sigma_{e e} r_{i}},
$$

e

$$
U_{i \beta \beta}^{*}=\frac{\partial^{2} \ell_{i}^{*}}{\partial^{2} \beta}=-s_{i} \bar{X}_{i}^{2} \sigma_{e e}^{-1}+\frac{\sigma_{u u} s_{i}}{\sigma_{e e} r_{i}} .
$$

Derivadas com relação a $\beta$ e $\sigma_{e e}$

$$
U_{i \beta \sigma_{e e}}^{*}=\frac{\partial^{2} \ell_{i}^{*}}{\partial \sigma_{e e} \partial \beta}=-s_{i} \bar{X}_{i} \sigma_{e e}^{-2}\left\{\bar{Y}_{i}-\alpha-\beta \bar{X}_{i}\right\}-\frac{\sigma_{u u} \beta s_{i}}{\sigma_{e e}^{2} r_{i}},
$$

similarmente para $\sigma_{e e}$

$$
U_{i \sigma_{e e}}^{*}=\frac{\partial \ell_{i}^{*}}{\partial \sigma_{e e}}=-\frac{s_{i}}{2 \sigma_{e e}}+\frac{1}{2 \sigma_{e e}^{2}} \sum_{j=1}^{s_{i}}\left(Y_{i j}-\alpha-\beta \bar{X}_{i}\right)^{2}-\frac{\beta^{2} \sigma_{u u} s_{i}}{2 \sigma_{e e}^{2} r_{i}},
$$

e

$$
U_{i \sigma_{e e} \sigma_{e e}}^{*}=\frac{\partial^{2} \ell_{i}^{*}}{\partial \sigma_{e e} \partial \sigma_{e e}}=\frac{s_{i}}{2 \sigma_{e e}^{2}}-\frac{1}{\sigma_{e e}^{3}} \sum_{j=1}^{s_{i}}\left(Y_{i j}-\alpha-\beta \bar{X}_{i}\right)^{2}+\frac{\beta^{2} \sigma_{u u} s_{i}}{\sigma_{e e}^{3} r_{i}}
$$

Assim, comprova-se que as derivadas são permutáveis, então:

$$
\begin{aligned}
& U_{i \sigma_{e e} \beta}^{*}=U_{i \sigma_{e e} \beta}^{*} \quad e \\
& U_{i \sigma_{e e} \alpha}^{*}=U_{i \alpha \sigma_{e e}}^{*} .
\end{aligned}
$$


Portanto, com

$$
U_{i}^{*}(\theta)=\left[\begin{array}{c}
\sigma_{e e}^{-1}\left\{s_{i} \bar{Y}_{i}-s_{i} \alpha-\beta \bar{X}_{i} s_{i}\right\} \\
s_{i} \bar{X}_{i} \sigma_{e e}^{-1}\left\{\bar{Y}_{i}-\alpha-\beta \bar{X}_{i}\right\}+\frac{\sigma_{u u} \beta s_{i}}{\sigma_{e e} r_{i}} \\
-\frac{s_{i}}{2 \sigma_{e e}}+\frac{1}{2 \sigma_{e e}^{2}} \sum_{j=1}^{s_{i}}\left(Y_{i j}-\alpha-\beta \bar{X}_{i}\right)^{2}-\frac{\beta^{2} \sigma_{u u} s_{i}}{2 \sigma_{e e}^{2} r_{i}}
\end{array}\right]
$$

e $U_{i}^{*}(\theta)^{\prime}$ obtem-se $\bar{s}_{n}^{*}(\theta)=\frac{1}{n} \sum_{i=1}^{n} U_{i}^{*}(\theta) U_{i}^{*}(\theta)^{\prime}$.

E para $I_{i k l}^{*}(\theta)=-\frac{\partial}{\partial \theta_{l}} U_{i k}^{*}$, com $\mathrm{k}=\mathrm{l}=\left(\alpha, \beta, \sigma_{e e}\right)$ tem-se

$$
I_{i}^{*}(\theta)=\left[\begin{array}{ccc}
I_{i \alpha \alpha} & I_{i \alpha \beta} & I_{i \alpha \sigma_{e e}} \\
& I_{i \beta \beta} & I_{i \beta \sigma_{e e}} \\
& & I_{i \sigma_{e e} \sigma_{e e}}
\end{array}\right]
$$

onde

$$
\begin{aligned}
& I_{i \alpha \alpha}=-s_{i} \sigma_{e e}^{-1}, \\
& I_{i \alpha \beta}=-s_{i} \bar{X}_{i} \sigma_{e e}^{-1}, \\
& I_{i \alpha \sigma_{e e}}=-s_{i} \sigma_{e e}^{-2}\left\{\bar{Y}_{i}-\alpha-\beta \bar{X}_{i}\right\} \\
& I_{i \beta \beta}=-s_{i} \bar{X}_{i}^{2} \sigma_{e e}^{-1}+\frac{\sigma_{u u} s_{i}}{\sigma_{e e} r_{i}}, \\
& I_{i \beta \sigma_{e e}}=-s_{i} \bar{X}_{i} \sigma_{e e}^{-2}\left(\bar{Y}_{i}-\alpha-\beta \bar{X}_{i}\right)-\frac{\beta \sigma_{u u} s_{i}}{\sigma_{e e}^{2} r_{i}} e \\
& I_{i \sigma_{e e} \sigma_{e e}}=\frac{s_{i}}{2 \sigma_{e e}}-\frac{1}{\sigma_{e e}^{3}} \sum_{j=1}^{s_{i}}\left(\bar{Y}_{i}-\alpha-\beta \bar{X}_{i}\right)^{2}+\frac{\beta^{2} \sigma_{u u} s_{i}}{\sigma_{e e}^{3} r_{i}},
\end{aligned}
$$

Desta maneira, pode-se obter $\bar{I}_{n}^{*}(\theta)=\frac{1}{n} \sum_{i=1}^{n} I_{i}^{*}(\theta)$. Finalmente o estimador consistente de $\theta$, obtém-se substituíndo as respetivas expresões em $\left\{\bar{I}_{n}^{*}(\theta)\right\}^{-1} \bar{s}_{n}^{*}(\theta)\left\{\bar{I}_{n}^{*}(\theta)^{\prime}\right\}^{-1}$. 


\subsection{Aplicação do método quando $\lambda=\frac{\sigma_{e e}}{\sigma_{u u}}$ conhecido}

Nesta seção, encontram-se os estimadores obtidos pelo método do escore corrigidos proposto por Gimenez (1997), pois mediante o método de Nakamura (1990) não é possível, quando $\lambda=\frac{\sigma_{e e}}{\sigma_{u u}}$ é conhecido. Obteve-se a função $U(\theta)$ desde a equação (2.4), onde

$$
U_{i}(\theta)=\left[\begin{array}{c}
\sigma_{e e}^{-1}\left\{s_{i} \bar{Y}_{i}-s_{i} \alpha-\beta x_{i} s_{i}\right\} \\
s_{i} x_{i} \sigma_{e e}^{-1}\left\{\bar{Y}_{i}-\alpha-\beta x_{i}\right\} \\
-\frac{s_{i}}{2 \sigma_{e e}}+\frac{1}{2 \sigma_{e e}^{2}} \sum_{j=1}^{s_{i}}\left(Y_{i j}-\alpha-\beta x_{i}\right)^{2}
\end{array}\right]
$$

sendo $U(\theta)=\sum_{i=1}^{n} U_{i}(\theta)$.

Considerando $U^{*}(\theta)=\sum_{\mathbf{i}=\mathbf{1}}^{\mathbf{n}} \mathbf{U}_{\mathbf{i}}^{*}(\theta)$ com

segue que

$$
U_{i}^{*}(\theta)=\left[\begin{array}{c}
\sigma_{e e}^{-1}\left\{s_{i} \bar{Y}_{i}-s_{i} \alpha-\beta \bar{X}_{i} s_{i}\right\} \\
s_{i} \bar{X}_{i} \sigma_{e e}^{-1}\left\{\bar{Y}_{i}-\alpha-\beta \bar{X}_{i}\right\}+\lambda^{-1} \beta \frac{s_{i}}{r_{i}} \\
-\frac{s_{i}}{2 \sigma_{e e}}+\frac{1}{2 \sigma_{e e}^{2}} \sum_{j=1}^{s_{i}}\left(Y_{i j}-\alpha-\beta \bar{X}_{i}\right)^{2}-\lambda^{-1} \sigma_{e e}^{-1} \beta^{2} \frac{s_{i}}{2 r_{i}}
\end{array}\right]
$$

$$
E\left[U_{i}^{*}(\theta ; \mathbf{X}, \mathbf{Y}) \mid \mathbf{x}_{\mathbf{i}}, \mathbf{Y}\right]=\mathbf{U}\left(\theta ; \mathbf{x}_{\mathbf{i}}, \mathbf{Y}\right), \quad \mathbf{i}=\mathbf{1}, \mathbf{2}, \ldots, \mathbf{n}
$$

e portanto, $U^{*}(\theta ; \mathbf{X}, \mathbf{Y})=\sum_{\mathbf{i}=\mathbf{1}}^{\mathbf{n}} \mathbf{U}_{\mathbf{i}}^{*}(\theta ; \mathbf{X}, \mathbf{Y})$ é um escore corrigido. Neste caso, o logaritmo da função de verossimilhança corrigida não existe, ou seja, não existe uma função $\ell^{*}(\theta ; \mathbf{X}, \mathbf{Y})$ tal que $E\left[\ell^{*}(\theta ; \mathbf{X}, \mathbf{Y}) \mid \mathbf{x}, \mathbf{Y}\right]=\ell(\theta ; \mathbf{x}, \mathbf{Y})$ e que $E\left[U_{i}^{*}(\theta ; \mathbf{X}, \mathbf{Y}) \mid \mathbf{x}_{\mathbf{i}}, \mathbf{Y}\right]=$ $\mathbf{U}\left(\theta ; \mathbf{x}_{\mathbf{i}}, \mathbf{Y}\right), \mathrm{i}=1,2, \ldots, \mathrm{n}$.

Resolvendo a equação $U^{*}(\theta ; \mathbf{X}, \mathbf{Y})=\mathbf{0}$, obtemos os estimadores 
3.4 Aplicação do método quando $\lambda=\frac{\sigma_{e e}}{\sigma_{u u}}$ conhecido

$\widehat{\theta}=\left(\widehat{\alpha}^{*}, \widehat{\beta}^{*}, \widehat{\sigma}_{e e}^{*}, \widehat{\sigma}_{u u}^{*}\right)^{\prime}$ como solução do seguinte sistema de equações:

$$
\begin{gathered}
\widehat{\alpha}^{*}=\frac{\sum_{i=1}^{n} s_{i} \bar{Y}_{i}-\widehat{\beta}^{*} \sum_{i=1}^{n} s_{i} \bar{X}_{i}}{\sum_{i=1}^{n} s_{i}}, \\
\widehat{\beta}^{*}=\frac{\sum_{i=1}^{n} s_{i} \bar{X}_{i} \bar{Y}_{i}-\left(\frac{\sum_{i=1}^{n} s_{i} \bar{Y}_{i}}{\sum_{i=1}^{n} s_{i}}\right)\left(\sum_{i=1}^{n} s_{i} \bar{X}_{i}\right)}{\sum_{i=1}^{n} s_{i} \bar{X}_{i}^{2}-\widehat{\sigma}_{e e}^{*} \lambda^{-1} \sum_{i=1}^{n} \frac{s_{i}}{r_{i}}-\frac{\left(\sum_{i=1}^{n} s_{i} \bar{X}_{i}\right)^{2}}{\sum_{i=1}^{n} s_{i}}}, \\
\widehat{\sigma}_{e e}^{*}=\frac{\sum_{i=1}^{n} \sum_{j=1}^{s_{i}}\left(Y_{i j}-\widehat{\alpha}^{*}-\widehat{\beta}^{*} \bar{X}_{i}\right)^{2}}{\sum_{i=1}^{n} s_{i}+\lambda^{-1}\left(\widehat{\beta}^{*}\right)^{2} \sum_{i=1}^{n} \frac{s_{i}}{r_{i}}} e \\
\widehat{\sigma}_{u u}^{*}=\lambda^{-1} \widehat{\sigma}_{e e}^{*} .
\end{gathered}
$$

Para sair da convergência iterativa, utilizou-se uma serie de operações algébricas descritas a seguir. Fazendo $\sigma_{e e}=\phi$ na segunda equação de $U_{i}^{*}(\theta)$, tem-se:

$$
\sum_{i=1}^{n} s_{i} \bar{X}_{i} \phi^{-1}\left\{\bar{Y}_{i}-\alpha-\beta \bar{X}_{i}\right\}+\lambda^{-1} \beta \sum_{i=1}^{n} \frac{s_{i}}{r_{i}}=0 .
$$

Resolvendo a equação (3.40) pode-se expressar como

$$
\phi^{-1}\left[S_{X Y}-\beta S_{X}^{2}\right]+\lambda^{-1} \beta \sum_{i=1}^{n} \frac{s_{i}}{r_{i}}=0
$$

onde $S_{X Y}=\sum_{i=1}^{n} s_{i}\left(\bar{X}_{i}-\bar{X}_{\bullet}\right)\left(\bar{Y}_{i}-\bar{Y}_{\bullet}\right), S_{X}^{2}=\sum_{i=1}^{n} s_{i}\left(\bar{X}_{i}-\bar{X}_{\bullet}\right)^{2}, S_{Y}^{2}=$ $\sum_{i=1}^{n} \sum_{j=1}^{s_{i}}\left(Y_{i j}-\bar{Y}_{\bullet}\right)^{2}, \bar{X}_{\bullet}=\sum_{i=1}^{n} \frac{s_{i} \bar{X}_{i}}{s_{i}}, \bar{Y} \bullet=\sum_{i=1}^{n} \frac{s_{i} \bar{Y}_{i}}{s_{i}}, \bar{X}_{i}=\sum_{k=1}^{r_{i}} \frac{X_{i k}}{r_{i}}, \bar{Y}_{i}=$ $\sum_{j=1}^{s_{i}} \frac{Y_{i j}}{s_{i}}, \widehat{\alpha}^{*}=\bar{Y}_{\bullet}-\widehat{\beta}^{*} \bar{X}_{\bullet}$. 
3.4 Aplicação do método quando $\lambda=\frac{\sigma_{e e}}{\sigma_{u u}}$ conhecido

Similarmente a equação (3.38) pode expressar-se como:

$$
\phi=\frac{\sum_{i=1}^{n} \sum_{j=1}^{s_{i}}\left(Y_{i j}-\widehat{\alpha}^{*}-\widehat{\beta}^{*} \bar{X}_{i}\right)^{2}}{\sum_{i=1}^{n} s_{i}+\lambda^{-1}\left(\widehat{\beta}^{*}\right)^{2} \sum_{i=1}^{n} \frac{s_{i}}{r_{i}}}
$$

Substituindo a equação (3.42) em (3.41), obteve-se uma equação de segundo grau em função de de $\widehat{\beta}^{*}$

$$
{\widehat{\beta^{*}}}^{2} S_{X Y} \lambda^{-1} \sum_{i=1}^{n} \frac{s_{i}}{r_{i}}-\widehat{\beta^{*}}\left(S_{Y}^{2} \lambda^{-1} \sum_{i=1}^{n} \frac{s_{i}}{r_{i}}-S_{X}^{2} \sum_{i=1}^{n} s_{i}\right)-S_{X Y} \sum_{i=1}^{n} s_{i}=0
$$

Portanto, a raiz positiva de $\beta$ esta representada por 


$$
\begin{gathered}
\widehat{\beta}^{*}=\frac{-\left(S_{Y}^{2} \lambda^{-1} \sum_{i=1}^{n} \frac{s_{i}}{r_{i}}-S_{X}^{2} \sum_{i=1}^{n} s_{i}\right)}{2 S_{X Y} \lambda^{-1} \sum_{i=1}^{n} \frac{s_{i}}{r_{i}}} \\
+\frac{\sqrt{\left(S_{Y}^{2} \lambda^{-1} \sum_{i=1}^{n} \frac{s_{i}}{r_{i}}-S_{X}^{2} \sum_{i=1}^{n} s_{i}\right)^{2}+4\left(S_{X Y} \sum_{i=1}^{n} s_{i}\right)\left(S_{X Y} \lambda^{-1} \sum_{i=1}^{n} \frac{s_{i}}{r_{i}}\right)}}{2 S_{X Y} \lambda^{-1} \sum_{i=1}^{n} \frac{s_{i}}{r_{i}}} .
\end{gathered}
$$

\subsubsection{Cálculos para encontrar a matriz de covariâncias as- sintótica}

Considerando a expresão da variância assintótica para o método escore corrigido, é necessário a funcão escore corrigida $U_{i}^{*}(\theta)$ :

$$
U_{i}^{*}(\theta)=\left[\begin{array}{c}
\sigma_{e e}^{-1}\left\{s_{i} \bar{Y}_{i}-s_{i} \alpha-\beta \bar{X}_{i} s_{i}\right\} \\
s_{i} \bar{X}_{i} \sigma_{e e}^{-1}\left\{\bar{Y}_{i}-\alpha-\beta \bar{X}_{i}\right\}+\lambda^{-1} \beta \frac{s_{i}}{r_{i}} \\
-\frac{s_{i}}{2 \sigma_{e e}}+\frac{1}{2 \sigma_{e e}^{2}} \sum_{j=1}^{s_{i}}\left(Y_{i j}-\alpha-\beta \bar{X}_{i}\right)^{2}-\lambda^{-1} \sigma_{e e}^{-1} \beta^{2} \frac{s_{i}}{2 r_{i}}
\end{array}\right]
$$

e $U_{i}^{*}(\theta)^{\prime}$ para obter $\bar{s}_{n}^{*}(\theta)=\frac{1}{n} \sum_{i=1}^{n} U_{i}^{*}(\theta) U_{i}^{*}(\theta)^{\prime}$.

E com relação a $\theta_{l}$, o $I_{i k l}^{*}(\theta)=-\frac{\partial}{\partial \theta_{l}} U_{i k}^{*}$, com $\mathrm{k}=\mathrm{l}=\left(\alpha, \beta, \sigma_{e e}\right)$ obtém-se

$$
I_{i}^{*}(\theta)=\left[\begin{array}{ccc}
I_{i \alpha \alpha} & I_{i \alpha \beta} & I_{i \alpha \sigma_{e e}} \\
& I_{i \beta \beta} & I_{i \beta \sigma_{e e}} \\
& & I_{i \sigma_{e e} \sigma_{e e}}
\end{array}\right]
$$


onde

$$
\begin{aligned}
& I_{i \alpha \alpha}=-s_{i} \sigma_{e e}^{-1}, \\
& I_{i \alpha \beta}=-s_{i} \bar{X}_{i} \sigma_{e e}^{-1}, \\
& I_{i \alpha \sigma_{e e}}=-s_{i} \sigma_{e e}^{-2}\left\{\bar{Y}_{i}-\alpha-\beta \bar{X}_{i}\right\}, \\
& I_{i \beta \beta}=-s_{i} \bar{X}_{i}^{2} \sigma_{e e}^{-1}+\lambda^{-1} \frac{s_{i}}{r_{i}}, \\
& I_{i \beta \sigma_{e e}}=-s_{i} \bar{X}_{i} \sigma_{e e}^{-2}\left(\bar{Y}_{i}-\alpha-\beta \bar{X}_{i}\right)-\lambda^{-1} \sigma_{e e}^{-1} \beta \frac{s_{i}}{r_{i}}, \\
& I_{i \sigma_{e e} \sigma_{e e}}=\frac{s_{i}}{2 \sigma_{e e}^{2}}-\frac{1}{\sigma_{e e}^{3}} \sum_{j=1}^{s_{i}}\left(Y_{i j}-\alpha-\beta \bar{X}_{i}\right)^{2}+\lambda^{-1} \sigma_{e e}^{-2} \beta^{2} \frac{s_{i}}{2 r_{i}},
\end{aligned}
$$

Portanto, pode-se obter $\bar{I}_{n}^{*}(\theta)=\frac{1}{n} \sum_{i=1}^{n} I_{i}^{*}(\theta)$. Finalmente o estimador para a matriz de covariâncias assintótica, obtém-se substituindo as respetivas expresões em $\left\{\bar{I}_{n}^{*}(\theta)\right\}^{-1} \bar{s}_{n}^{*}(\theta)\left\{\bar{I}_{n}^{*}(\theta)^{\prime}\right\}^{-1}$. Cálculos para encontrar a matriz de covariâncias assintótica Sem perda de generalidade na Seção 3.4, pode-se obter os resultados quando $\sigma_{e e}=\sigma_{u u}=\sigma$, utilizando $\lambda=1$.

Sabe-se, também que:

$$
\begin{aligned}
& \bar{U}_{n}^{*}(\theta)=\frac{1}{n} \bar{U}^{*}(\theta)=\frac{1}{n} \sum_{i=1}^{n} U_{i}^{*}(\theta), \\
& \bar{I}_{n}^{*}(\theta)=\frac{1}{n} \bar{I}^{*}(\theta)=\frac{1}{n} \sum_{i=1}^{n} I_{i}^{*}(\theta), \\
& \bar{\Lambda}_{n}(\theta)=\frac{1}{n} E\left[\bar{I}^{*}(\theta)\right]=\frac{1}{n} \sum_{i=1}^{n} E\left[I_{i}^{*}(\theta)\right], \\
& \bar{\Gamma}_{n}(\theta)=\frac{1}{n} E\left[U_{i}^{*}(\theta) U_{i}^{*}(\theta)^{\prime}\right],
\end{aligned}
$$

pode-se obter $\left\{\bar{I}_{n}^{*}(\theta)\right\}^{-1} \bar{s}_{n}^{*}(\theta)\left\{\bar{I}_{n}^{*}(\theta)^{\prime}\right\}^{-1}$ é um estimador consistente de

$$
\left\{\bar{\Lambda}_{n}\left(\theta_{0}\right)\right\}^{-1} \bar{\Gamma}_{n}\left(\theta_{0}\right)\left\{\bar{\Lambda}_{n}\left(\theta_{0}\right)^{\prime}\right\}^{-1} \text {. }
$$




\section{Capítulo 4}

\section{Comparação entre os métodos de estimação}

Neste capítulo apresenta-se um estudo de simulação para comparação dos métodos de máxima verossimilhança e do escore corrigido onde são calculados o vício e o erro quadrático médio empíricos dos estimadores para as amostras geradas.

\subsection{Simulações}

Realizou-se um estudo de simulação para investigar o desempenho dos métodos de estimação descritos nos capítulos anteriores. A seguir, são mostrados os resultados numéricos nos quais foram comparados a estimação pelo método de máxima verossimilhança com a estimação realizada através do método do escore corrigido. Usando o critério de acurácia, que mede a proximidade de cada observação em relação ao valor alvo que se procura atingir, e de precisão, que mede a proximidade de cada observação em relação a média de todas as observações, será utilizado o erro quadrático 
médio empírico (EQM E) e o viés empírico (BE) para comparação dos parâmetros estimados.

Ressalta-se aqui que o viés empírico $(\mathrm{BE})$ e o erro quadrático médio empírico (EQM E) para um parâmetro, são obtidos através das expressões:

$$
B E=\frac{\sum_{i=1}^{N A}\left(\widehat{\theta}_{i}-\theta\right)}{N A}
$$

$\mathrm{e}$

$$
E Q M E=\frac{\sum_{i=1}^{N A}\left(\widehat{\theta}_{i}-\theta\right)^{2}}{N A}
$$

A soma nas expressões acima (4.1) e (4.2) se estende a todas as amostras simuladas.

Os valores dos parâmetros utilizados foram $\alpha=1 ; \beta=1 ; \sigma_{u u}=1, \sigma_{e e}=$ $\{1,2,3\}$, para vários valores de $n=\{3,15,30\}$ observando o comportamento dos parâmetros estimados. As réplicas que variam independentemente para cada unidade de observação serão mostradas nas tabelas apresentadas adiante.

Para gerar as simulações, considerou-se os valores de $x_{i} \sim N\left(\mu_{x} ; \sigma_{x x}\right)$, com $\mu_{x}=1$ e $\sigma_{x x}=10$, usando diferentes valores do tamanho da unidade em observação com suas respectivas réplicas para cada unidade observada. Utilizando o método de Monte Carlos (MC) com 5000 amostras simuladas, obtém-se os resultados mostrados a seguir.

\subsubsection{Resultados}

As tabelas que seguem mostram parte dos resultados das simulações para amostras de tamanho 3 (Tabelas 4.1, 4.2 e 4.3), tamanho 15 (Tabelas 4.4, 4.5 e 4.6) e tamanho 30 (Tabelas 4.7, 4.8 e 4.9). Estas tabelas permitem a comparação de estimadores 
utilizando o viés e erro quadrático médio empírico. Os resultados obtidos através da simulação realizada pelo método de máxima verossimilhança corrigida, no qual os estimadores dos parâmetros estruturais $\alpha, \beta, \sigma_{e e}$ e $\sigma_{u u}$ estimados, apresentaram pequenos viés e erro quadrático médio. Verifica-se que estes estimadores são menos viciados, ou seja, mais precisos e eficientes.

De forma semelhante, os estimadores para obter $\alpha, \beta, \sigma_{e e}$ através do método escore corrigido, quando $\sigma_{u u}$ é conhecido, mostraram pequenos vies e erro quadrático médio, mas, maiores do que os obtidos pelo método máxima verossimilhança. Durante a simulação pelo método escore corrigido onde o valor de $\sigma_{u u}$ não é conhecido, a aplicação deste método torna-se factível substituindo o valor de $\sigma_{u u}$ pelo valor de $\sigma_{u u}$ estimado pelas réplicas, equação (3.23). Assim, os resultados da simulação comparando os dois métodos foram semelhantes, observando apenas uma pequena diferença nos decimais dos valores de viés e erro quadrático médio, descritos nas Tabelas 4.1 a 4.9 .

Um resultado diferente é encontrado quando utiliza-se o método do escore corrigido onde $\lambda=\frac{\sigma_{e e}}{\sigma_{u u}}$ é conhecido. Isto é observado nas Tabelas 4.1 a 4.9, onde o viés e o erro quadrático médio empírico são maiores.

Cabe ressaltar que ao utilizar $\sigma_{u u}$ estimado, os resultados das simulações são os mesmos, não obtendo muita diferença entre as estimativas.

Analizando todas as simulações mostradas nas tabelas abaixo, pode-se concluir que a medida que aumenta o tamanho da amostra, os dois métodos de estimação (máxima verossimilhança corrigido e escore corrigido onde $\sigma_{u u}$ é conhecido) são similares e geram bons estimadores. Assim, é possivel estabelecer a seguinte relação:

$$
\operatorname{EQME}\left(\widehat{\theta}_{M V}\right)<\operatorname{EQME}\left(\widehat{\theta}_{S C \sigma_{u u} \text { conhecido }}\right)<\operatorname{EQME}\left(\widehat{\theta}_{S C \widehat{\sigma}_{u u}}\right)<\operatorname{EQME}\left(\widehat{\theta}_{\text {SCAconhecido }}\right) .
$$


Tabela 4.1: Vício e erro quadrático médio empírico, com tamanho de amostra $n=3$, réplicas de valores $r=(16,20,30)$ e $s=(8,10,15)$.

\begin{tabular}{|c|c|c|c|c|c|c|}
\hline & \multicolumn{2}{|c|}{ MV } & \multicolumn{4}{|c|}{$\mathrm{SC} \sigma_{u u}$ conhecido } \\
\hline Parâmetro & Vício E & EQM E & Vício E & EQM E & Vício E $\widehat{\sigma}_{u u}$ & $\mathrm{EQM} \mathrm{E} \widehat{\sigma}_{u u}$ \\
\hline$\alpha$ & $-0,1360507$ & 104,2831 & $-0,3188288$ & 932,1805 & $-2,014940$ & 3210,579 \\
\hline$\beta$ & 0,01552068 & 0,945661 & 0,03170343 & 9,770286 & 0,2028429 & 33,36878 \\
\hline$\sigma_{e e}$ & $-0,04142808$ & 0,06083417 & $-0,07953142$ & 0,1151248 & $-0,1938038$ & 0,5085695 \\
\hline \multirow[t]{7}{*}{$\sigma_{u u}$} & $-0,01218739$ & 0,03111794 & & & & \\
\hline & & & \multicolumn{2}{|c|}{$\mathrm{SC} \lambda$ conhecido } & & \\
\hline & & Parâmetro & Vício E & EQM E & & \\
\hline & & $\alpha$ & $-46,86133$ & 17549024 & & \\
\hline & & $\beta$ & 3,815094 & 130253,3 & & \\
\hline & & $\sigma_{e e}$ & 7,448605 & 116,6067 & & \\
\hline & & $\sigma_{u u}$ & 7,448605 & 116,6067 & & \\
\hline
\end{tabular}

Os valores verdadeiros utilizados na simulação foram: $\alpha=1, \beta=1, \sigma_{e e}=1, \sigma_{u u}=1$ e $x_{i} \sim N\left(\mu_{x} ; \sigma_{x x}\right), \operatorname{com} \mu_{x}=10$ e $\sigma_{x x}=3$. 
Tabela 4.2: Vício e erro quadrático médio empírico, com tamanho de amostra $n=3$, réplicas de valores $r=(16,20,30)$ e $s=(8,10,15)$.

\begin{tabular}{c|cc|cccc|}
\hline & \multicolumn{2}{|c|}{ MV } & \multicolumn{4}{c|}{ SC $\sigma_{u u}$ conhecido } \\
\hline Parâmetro & Vício E & EQM E & Vício E & EQM E & Vício E $\widehat{\sigma}_{u u}$ & EQM E $\widehat{\sigma}_{u u}$ \\
\hline$\alpha$ & $-0,1629844$ & 173,0274 & $-0,3445229$ & 1134,701 & $-2,282059$ & 4189,339 \\
$\beta$ & 0,01854214 & 1,632048 & 0,03364680 & 11,85464 & 0,2304810 & 43,46837 \\
$\sigma_{e e}$ & $-0,07534749$ & 0,2420704 & $-0,12799$ & 0,3281864 & $-0,2478048$ & 1,000479 \\
$\sigma_{u u}$ & $-0,01421825$ & 0,03101953 & & & & \\
\hline
\end{tabular}

\begin{tabular}{c|cc}
\hline & \multicolumn{2}{|c}{ SC $\lambda$ conhecido } \\
\hline Parâmetro & Vício E & EQM E \\
\hline$\alpha$ & 28,06969 & 504224,7 \\
$\beta$ & $-2,920764$ & 4907,481 \\
$\sigma_{e e}$ & 7,675299 & 126,6092 \\
$\sigma_{u u}$ & 3,837649 & 31,65231 \\
\hline
\end{tabular}

Os valores verdadeiros utilizados na simulação foram: $\alpha=1, \beta=1, \sigma_{e e}=2, \sigma_{u u}=1$ e $x_{i} \sim N\left(\mu_{x} ; \sigma_{x x}\right)$, com $\mu_{x}=10$ e $\sigma_{x x}=3$. 
Tabela 4.3: Vício e erro quadrático médio empírico, com tamanho de amostra $n=3$, réplicas de valores $r=(16,20,30)$ e $s=(8,10,15)$.

\begin{tabular}{c|cc|cccc|}
\hline & \multicolumn{2}{|c|}{ MV } & \multicolumn{4}{c|}{ SC $\sigma_{u u}$ conhecido } \\
\hline Parâmetro & Vício E & EQM E & Vício E & EQM E & Vício E $\widehat{\sigma}_{u u}$ & EQM E $\widehat{\sigma}_{u u}$ \\
\hline$\alpha$ & $-0,2095769$ & 222,0214 & $-0,3642387$ & 1312,775 & $-2,487026$ & 5184,415 \\
$\beta$ & 0,01991628 & 2,15071 & 0,03513799 & 13,68975 & 0,2516884 & 53,81012 \\
$\sigma_{e e}$ & $-0,1082083$ & 0,5431729 & $-0,1766152$ & 0,6638512 & $-0,3011126$ & 1,658623 \\
$\sigma_{u u}$ & $-0,01509051$ & 0,0309663 & & & & \\
\hline
\end{tabular}

\begin{tabular}{c|cc}
\hline & \multicolumn{2}{|c}{ SC $\lambda$ conhecido } \\
\hline Parâmetro & Vício E & EQM E \\
\hline$\alpha$ & 97,37485 & 31157315 \\
$\beta$ & $-8,775308$ & 237868,0 \\
$\sigma_{e e}$ & 7,789223 & 133,5011 \\
$\sigma_{u u}$ & 2,596408 & 14,83346 \\
\hline
\end{tabular}

Os valores verdadeiros utilizados na simulação foram: $\alpha=1, \beta=1, \sigma_{e e}=3, \sigma_{u u}=1$ e $x_{i} \sim N\left(\mu_{x} ; \sigma_{x x}\right)$, com $\mu_{x}=10$ e $\sigma_{x x}=3$. 
Tabela 4.4: Vício e erro quadrático médio empírico, com tamanho de amostra $n=15$, réplicas de valores $\mathrm{r}=(11,14,17,20,23,26,29,26,23,20,17,14,11,14,17)$ e $\mathrm{s}=(6,9,12,15,18,21,24,21,18,15,12,9,6,9,12)$.

\begin{tabular}{c|cc|cccc|}
\hline & \multicolumn{2}{|c|}{ MV } & \multicolumn{4}{c|}{ SC $\sigma_{u u}$ conhecido } \\
\hline Parâmetro & Vício E & EQM E & Vício E & EQM E & Vício E $\widehat{\sigma}_{u u}$ & EQM E $\widehat{\sigma}_{u u}$ \\
\hline$\alpha$ & $-0,008878329$ & 0,3718065 & $-0,01459396$ & 0,3807728 & $-0,6092244$ & 0,8010131 \\
$\beta$ & 0,0009564387 & 0,003613021 & 0,002050952 & 0,00365585 & 0,06148751 & 0,007926167 \\
$\sigma_{e e}$ & $-0,005011546$ & 0,01024843 & $-0,01112097$ & 0,01118658 & $-0,1761023$ & 0,04599539 \\
$\sigma_{u u}$ & $-0,00418253$ & 0,007412161 & & & & \\
\hline
\end{tabular}

\begin{tabular}{c|cc}
\hline & \multicolumn{2}{|c}{ SC $\lambda$ conhecido } \\
\hline Parâmetro & Vício E & EQM E \\
\hline$\alpha$ & 20,0264 & 402,344 \\
$\beta$ & $-2,003623$ & 4,018202 \\
$\sigma_{e e}$ & 10,62006 & 131,6162 \\
$\sigma_{u u}$ & 10,62006 & 131,6162 \\
\hline
\end{tabular}

Os valores verdadeiros utilizados na simulação foram: $\alpha=1, \beta=1, \sigma_{e e}=1, \sigma_{u u}=1$ e $x_{i} \sim N\left(\mu_{x} ; \sigma_{x x}\right)$, com $\mu_{x}=10$ e $\sigma_{x x}=3$. 
Tabela 4.5: Vício e erro quadrático médio empírico, com tamanho de amostra $n=15$, réplicas de valores $\mathrm{r}=(11,14,17,20,23,26,29,26,23,20,17,14,11,14,17)$ e $\mathrm{s}=(6,9,12,15,18,21,24,21,18,15,12,9,6,9,12)$.

\begin{tabular}{c|cc|cccc|}
\hline & \multicolumn{2}{|c|}{ MV } & \multicolumn{4}{c|}{ SC $\sigma_{u u}$ conhecido } \\
\hline Parâmetro & Vício E & EQM E & Vício E & EQM E & Vício E $\widehat{\sigma}_{u u}$ & EQM E $\widehat{\sigma}_{u u}$ \\
\hline$\alpha$ & $-0,003477321$ & 0,5852417 & $-0,002683522$ & 0,5989885 & $-0,5966369$ & 1,030240 \\
$\beta$ & 0,0004314768 & 0,005678497 & 0,0009285112 & 0,005741054 & 0,06030011 & 0,01013187 \\
$\sigma_{e e}$ & 0,01027581 & 0,04097107 & $-0,01560073$ & 0,04177807 & $-0,1805159$ & 0,07837963 \\
$\sigma_{u u}$ & $-0,01177115$ & 0,007418214 & & & & \\
\hline
\end{tabular}

\begin{tabular}{c|cc}
\hline & \multicolumn{2}{|c}{ SC $\lambda$ conhecido } \\
\hline Parâmetro & Vício E & EQM E \\
\hline$\alpha$ & 20,02434 & 402,4662 \\
$\beta$ & $-2,003443$ & 4,019568 \\
$\sigma_{e e}$ & 10,89590 & 139,3870 \\
$\sigma_{u u}$ & 5,447952 & 34,84676 \\
\hline
\end{tabular}

Os valores verdadeiros utilizados na simulação foram: $\alpha=1, \beta=1, \sigma_{e e}=2, \sigma_{u u}=1$ e $x_{i} \sim N\left(\mu_{x} ; \sigma_{x x}\right)$, com $\mu_{x}=10$ e $\sigma_{x x}=3$. 
Tabela 4.6: Vício e erro quadrático médio empírico, com tamanho de amostra $n=15$, réplicas de valores $\mathrm{r}=(11,14,17,20,23,26,29,26,23,20,17,14,11,14,17)$ e $\mathrm{s}=(6,9,12,15,18,21,24,21,18,15,12,9,6,9,12)$.

\begin{tabular}{c|cc|cccc|}
\hline & \multicolumn{3}{|c|}{ MV } & \multicolumn{4}{c|}{ SC $\sigma_{u u}$ conhecido } \\
\hline Parâmetro & Vício E & EQM E & Vício E & EQM E & Vício E $\widehat{\sigma}_{u u}$ & EQM E $\widehat{\sigma}_{u u}$ \\
\hline$\alpha$ & 0,0001407129 & 0,7993302 & 0,006455682 & 0,8184032 & $-0,5869782$ & 1,264360 \\
$\beta$ & 0,00008015013 & 0,007749905 & 0,0000672322 & 0,007841351 & 0,05938898 & 0,01238649 \\
$\sigma_{e e}$ & 0,02896723 & 0,09246756 & $-0,02004624$ & 0,09221553 & $-0,1849806$ & 0,1307716 \\
$\sigma_{u u}$ & $-0,01517178$ & 0,007439632 & & & & \\
\hline
\end{tabular}

\begin{tabular}{c|cc}
\hline & \multicolumn{2}{|c}{ SC $\lambda$ conhecido } \\
\hline Parâmetro & Vício E & EQM E \\
\hline$\alpha$ & 20,02247 & 402,5984 \\
$\beta$ & $-2,003276$ & 4,020992 \\
$\sigma_{e e}$ & 10,99637 & 142,8472 \\
$\sigma_{u u}$ & 3,665455 & 15,87191 \\
\hline
\end{tabular}

Os valores verdadeiros utilizados na simulação foram: $\alpha=1, \beta=1, \sigma_{e e}=3, \sigma_{u u}=1$ e $x_{i} \sim N\left(\mu_{x} ; \sigma_{x x}\right)$, com $\mu_{x}=10$ e $\sigma_{x x}=3$. 
Tabela 4.7: Vício e erro quadrático médio empírico, com tamanho de amostra $n=30$, réplicas de valores $\mathrm{r}=(11,14,17,20,23,26,29,26,23,20,17,14,11,14,17$, 20,23,26,29,26,23,20,17,14,11,14,17,20,23,26) e $\quad \mathrm{s}=(6,9,12,15,18,21,24,21$, $18,15,12,9,6,9,12,15,18,21,24,21,18,15,12,9,6,9,12,15,18,21)$.

\begin{tabular}{c|cc|cccc|}
\hline & \multicolumn{3}{|c|}{ MV } & \multicolumn{4}{c|}{ SC $\sigma_{u u}$ conhecido } \\
\hline Parâmetro & Vício E & EQM E & Vício E & EQM E & Vício E $\widehat{\sigma}_{u u}$ & EQM E $\widehat{\sigma}_{u u}$ \\
\hline$\alpha$ & $-0,01008605$ & 0,1601512 & $-0,02791004$ & 0,1571449 & $-0,5911162$ & 0,5253536 \\
$\beta$ & 0,001012805 & 0,001553072 & 0,002588823 & 0,001520118 & 0,05886711 & 0,005164401 \\
$\sigma_{e e}$ & $-0,001480800$ & 0,004852555 & $-0,006593019$ & 0,004893043 & $-0,1657136$ & 0,0345382 \\
$\sigma_{u u}$ & $-0,003282701$ & 0,003536909 & & & & \\
\hline
\end{tabular}

\begin{tabular}{c|cc}
\hline & \multicolumn{2}{|c}{ SC $\lambda$ conhecido } \\
\hline Parâmetro & Vício E & EQM E \\
\hline$\alpha$ & 20,00364 & 400,7596 \\
$\beta$ & $-2,002168$ & 4,010248 \\
$\sigma_{e e}$ & 10,97744 & 130,1835 \\
$\sigma_{u u}$ & 10,97744 & 130,1835 \\
\hline
\end{tabular}

Os valores verdadeiros utilizados na simulação foram: $\alpha=1, \beta=1, \sigma_{e e}=1, \sigma_{u u}=1$ e $x_{i} \sim N\left(\mu_{x} ; \sigma_{x x}\right)$, com $\mu_{x}=10$ e $\sigma_{x x}=3$. 
Tabela 4.8: Vício e erro quadrático médio empírico, com tamanho de amostra $n=30$, réplicas de valores $\mathrm{r}=(11,14,17,20,23,26,29,26,23,20,17,14,11,14,17$, $20,23,26,29,26,23,20,17,14,11,14,17,20,23,26)$ e $\quad \mathrm{s}=(6,9,12,15,18,21,24,21$, $18,15,12,9,6,9,12,15,18,21,24,21,18,15,12,9,6,9,12,15,18,21)$.

\begin{tabular}{c|cc|cccc|}
\hline & \multicolumn{2}{|c|}{ MV } & \multicolumn{4}{c|}{ SC $\sigma_{u u}$ conhecido } \\
\hline Parâmetro & Vício E & EQM E & Vício E & EQM E & Vício E $\widehat{\sigma}_{u u}$ & EQM E $\widehat{\sigma}_{u u}$ \\
\hline$\alpha$ & $-0,008343648$ & 0,2533729 & $-0,03560037$ & 0,2495617 & $-0,5992081$ & 0,6376692 \\
$\beta$ & 0,0008664437 & 0,002460651 & 0,003272155 & 0,002407806 & 0,05958938 & 0,006237011 \\
$\sigma_{e e}$ & 0,01790738 & 0,01970645 & $-0,01004205$ & 0,01823729 & $-0,1694692$ & 0,04934687 \\
$\sigma_{u u}$ & $-0,01110412$ & 0,003592253 & & & & \\
\hline
\end{tabular}

\begin{tabular}{c|cc}
\hline & \multicolumn{2}{|c}{$\mathrm{SC} \lambda$ conhecido } \\
\hline Parâmetro & Vício E & EQM E \\
\hline$\alpha$ & 20,00536 & 400,9217 \\
$\beta$ & $-2,002310$ & 4,011722 \\
$\sigma_{e e}$ & 11,25162 & 137,1901 \\
$\sigma_{u u}$ & 5,625812 & 34,29753 \\
\hline
\end{tabular}

Os valores verdadeiros utilizados na simulação foram: $\alpha=1, \beta=1, \sigma_{e e}=2, \sigma_{u u}=1$ e $x_{i} \sim N\left(\mu_{x} ; \sigma_{x x}\right)$, com $\mu_{x}=10$ e $\sigma_{x x}=3$. 
Tabela 4.9: Vício e erro quadrático médio empírico, com tamanho de amostra $n=30$, réplicas de valores $\mathrm{r}=(11,14,17,20,23,26,29,26,23,20,17,14,11,14,17$, $20,23,26,29,26,23,20,17,14,11,14,17,20,23,26)$ e $\quad \mathrm{s}=(6,9,12,15,18,21,24,21$, $18,15,12,9,6,9,12,15,18,21,24,21,18,15,12,9,6,9,12,15,18,21)$.

\begin{tabular}{c|cc|cccc|}
\hline & \multicolumn{2}{|c|}{ MV } & \multicolumn{4}{c|}{ SC $\sigma_{u u}$ conhecido } \\
\hline Parâmetro & Vício E & EQM E & Vício E & EQM E & Vício E $\widehat{\sigma}_{u u}$ & EQM E $\widehat{\sigma}_{u u}$ \\
\hline$\alpha$ & $-0,007517034$ & 0,3463983 & $-0,04150137$ & 0,3428525 & $-0,6054173$ & 0,7487334 \\
$\beta$ & 0,0008054031 & 0,003366291 & 0,003796494 & 0,003302506 & 0,06014359 & 0,007298317 \\
$\sigma_{e e}$ & 0,04090429 & 0,04511672 & $-0,01344856$ & 0,04019763 & $-0,1731424$ & 0,07275721 \\
$\sigma_{u u}$ & $-0,01462341$ & 0,003647201 & & & & \\
\hline
\end{tabular}

\begin{tabular}{c|cc}
\hline & \multicolumn{2}{|c}{$\mathrm{SC} \lambda$ conhecido } \\
\hline Parâmetro & Vício E & EQM E \\
\hline$\alpha$ & 20,00656 & 401,063 \\
$\beta$ & $-2,002406$ & 4,013017 \\
$\sigma_{e e}$ & 11,35114 & 140,0671 \\
$\sigma_{u u}$ & 3,783713 & 15,56301 \\
\hline
\end{tabular}

Os valores verdadeiros utilizados na simulação foram: $\alpha=1, \beta=1, \sigma_{e e}=3, \sigma_{u u}=1$ e $x_{i} \sim N\left(\mu_{x} ; \sigma_{x x}\right)$, com $\mu_{x}=10$ e $\sigma_{x x}=3$. 


\section{Capítulo 5}

\section{Considerações finais}

Neste trabalho apresentamos duas metodologias de estimação baseada nos métodos de máxima verossimilhança (Hokama, (2001)) e do escore corrigido (Gimenez, 1997), para o modelo de regressão linear simples funcional com erro normais e repetições não balanceadas. Apresentamos expressões para os estimadores dos parâmetros estruturais do modelo, que são $\alpha, \beta, \sigma_{e e}$ e $\sigma_{u u}$, assim como, expressões para a matriz de covariâncias assintótica, tanto para o método de máxima verossimilhança, quanto para o método escore corrigido quando $\sigma_{u u}$ e a razão das variâncias dos erros $\lambda=\sigma_{e e} / \sigma_{u u}$ são conhecidas. Aplicamos os resultados encontrados para realizar um estudo de simulação avaliando as propriedades dos estimadores e sua eficiência.

Os resultados dessas simulações mostram que, em geral, os vícios médios e os erros quadráticos médios dos estimadores obtidos pelo método de máxima verossimilhança são pequenos. Da mesma forma, os resultados obtidos com o método do escore corrigido com variância $\sigma_{u u}$ conhecido também mostram que os vícios médios e o erro quadrático médio são pequenos. Utilizando o estimador consistente $\widehat{\sigma}_{u u}$ calculado pelas repetições ao invés do parâmetro $\sigma_{u u}$ conhecido, obteve-se resultado 
semelhantes.

Analogicamente, quando utilizou-se o método do escore corrigido sendo $\lambda=$ $\sigma_{e e} / \sigma_{u u}$ conhecida, em geral, os resultados obtidos mostraram que os vícios médios e os erros quadráticos médios dos parâmetros estruturais do modelo são grandes. Isso deve-se ao estimador de $\beta$ encontrado em (3.44), pois não é um estimador robusto.

Finalmente, notamos que no modelo de regressão linear simples funcional com erro normais e repetições não balanceadas, pode-se concluir que a medida que aumenta o tamanho da amostra, os dois métodos de estimação, resultados dos Capítulos 2 e 3.3, tanto de máxima verossimilhança quanto do escore corrigido onde $\sigma_{u u}$ é estimado são similares e geram bons estimadores. Cabe ressaltar que o tamanho das repetições independem do resultado da estimativa dos métodos utilizados. 


\section{Apêndice A}

\section{Condições de regularidade}

As condições de regularidade para a validade das propriedades assintóticas dos estimadores do escore corrigido e de máxima verossimilhança no modelo funcional são estudadas a seguir. Sendo as seguintes derivadas

$$
I_{i k l}^{*}\left(\theta ; x_{i}, y_{i}\right)=-\frac{\partial U_{i k}^{*}}{\partial \theta_{l}}, \quad k=l=1,2, \ldots, n \text { exitem quase certamente, } \forall i
$$

Para o modelo apresentado tem-se $k=l=\left(\alpha, \beta, \sigma_{e e}, \sigma_{u u}\right)$

C1. Para cada $\theta \in F$, existe $\delta>0$ e uma função $d_{i k l}\left(=d_{i k l}\left(x_{i}, y_{i}\right)\right.$ tal que

$$
\left|I_{i k l}^{*}\left(\theta^{*}\right)\right|<d_{i k l}, \quad q . c
$$

$i=1.2, \ldots, n ; k=l=1,2, \ldots, p, \forall \theta^{*} \in B_{\theta, \delta}=\left\{\theta^{*}:\left\|\theta^{*}-\theta\right\|<\delta\right\} \subset F, \mathrm{e}$

$$
0<\lim \sup \frac{1}{n} \sum_{i=n}^{n} E\left[d_{i k l}^{2}\right]<\infty .
$$

C2. $0<\lim \inf \operatorname{det}\left(\bar{\Lambda}_{n}\left(\theta_{0}\right)\right), 0<\lim \inf \left\|\left(\bar{\Lambda}_{n}^{-1}\left(\theta_{0}\right)\right)\right\| \leq \lim \sup \left\|\left(\bar{\Lambda}_{n}^{-1}\left(\theta_{0}\right)\right)\right\|<\infty$, onde para uma matriz $A=\left[a_{i j}\right],\|A\|=\left(\sum_{i=1}^{p} \sum_{j=1}^{p} a_{i j}^{2}\right)^{1 / 2} \mathrm{e} \operatorname{det}(A) \operatorname{denota} \mathrm{o}$ determinante de $A$. 
C3. Dado $\epsilon>0$, existe $\delta>0$ tal que

$$
\lim \sup \frac{1}{n}\left|\sum_{i=1}^{n} E\left[\sup _{\theta \in B_{\theta_{0} ; \delta}} I_{i k l}^{*}(\theta)-I_{i k l}^{*}\left(\theta_{0}\right)\right]\right|<\epsilon,
$$

$k, l=1,2, \ldots, p$. Uma condição análoga é assumida com lim inf no lugar de lim sup.

C4. Existe $\gamma>0$ tal que, quando $n \rightarrow \infty$,

$$
\frac{1}{n^{1+\frac{\gamma}{2}}} \sum_{i=1}^{n} E\left[\left|U_{i k}^{*}\left(\theta_{0}\right)\right|\right]^{2+\gamma} \rightarrow 0, \quad k=1,2, \ldots, p .
$$

C5. $0<\lim \inf \frac{1}{n} \sum_{i=1}^{n} E\left[U_{i k}^{*}\left(\theta_{0}\right) U_{i l}^{*}\left(\theta_{0}\right)\right] \leq \lim \sup \frac{1}{n} \sum_{i=1}^{n} E\left[U_{i k}^{*}\left(\theta_{0}\right) U_{i l}^{*}\left(\theta_{0}\right)\right]<\infty$, $k, l=1,2, \ldots, p$.

\section{Observações}

1. Se as segundas derivadas parciais de $U_{i k}^{*}\left(\theta_{0}\right)$ existem q.c., então a condição C3 pode ser substituída por C3*, podendo ser verificadas em algumas situações, C3*. Existe uma função $d_{i k l}\left(=d_{i k l}\left(x_{i}, y_{i}\right)\right.$ tal que

$$
\begin{array}{r}
\left|\frac{\partial^{2} U_{i k}^{*}(\theta)}{\partial \theta_{l} \partial \theta_{m}}\right|<d_{i k l m} \quad q . c ., \\
k=l=m=1,2, \ldots, p, i=1,2, \ldots, n \forall \theta \in B_{\theta_{0}, \delta} \text { e } \\
\lim \sup \frac{1}{n} \sum_{i=1}^{n} E\left[\left|d_{i k l m}\right|\right]<\infty
\end{array}
$$

2. Num modelo funcional, as propriedades assintóticas do estimador do escore corrigido claramente dependerão do comportamento assintótico dos $x_{i 0}$, verdadeiros $x_{i}$, anunciados pelas condições $\mathrm{C} 1$ - C5, desde que as matrizes envolvidas dependem de $x_{i 0}$. Note que não é assumido que as matrizes $\bar{\Lambda}$ e $\bar{\Gamma}$ convergem a nenhum limite. As condições $\mathrm{C} 2$ e C5, respectivamente, determinam o comportamento assintótico desta matriz. 
3. A Condição C4 é estabelecida para a aplicação do Teorema Central do Limite de Liaponov. 


\section{Apêndice B}

\section{O Método Delta}

O método delta é bastante utilizado para a obtenção da distribuição assintótica de funções de variáveis aleatórias.

Teorema B.1 Sejam $\left\{T_{n}\right\}_{n \geq 1}=\left\{\left(T_{n 1}, T_{n 2}, \ldots, T_{n p}\right)\right\}_{n \geq 1}$ uma seqüência de vetores aleatórios p-dimencionais, $\mu=\left(\mu_{1}, \mu_{2}, \ldots, \mu_{p}\right) \in R^{p}$ e $g(x)=\left(x_{1}, x_{2}, \ldots, x_{p}\right)$ uma função com valores reais tal que $g_{j}^{\bullet}=\frac{\partial g(x)}{\partial x_{j}}$ existe em uma vizinhança de $\mu$ e é continua no ponto $\mu$, para $j=1,2, \ldots, p$. Seja

$$
g^{\bullet}(u)=\left(g_{j}^{\bullet}(u)\right)_{1 \leq j \leq p}=\left(\left.\frac{\partial g(x)}{\partial x_{j}}\right|_{x=\mu}\right)_{1 \leq j \leq p}
$$

$S e \sqrt{n}\left(T_{n}-\mu\right) \stackrel{D}{\rightarrow} N(0, \Sigma) \quad$ ent $\tilde{a} o, \sqrt{n}\left(g\left(T_{n}\right)-g(\mu)\right) \stackrel{D}{\rightarrow} N\left(0, g^{\bullet}\left(T_{n}\right) \Sigma g^{\bullet}\left(T_{n}\right)^{t}\right)$ Demonstração: Ver Serfling (1980). 


\section{Apêndice C}

\section{Programas em R}

Nesta seção apresentam-se os programas em R para a implementação do método iterativo de MV e do escore corrigido.

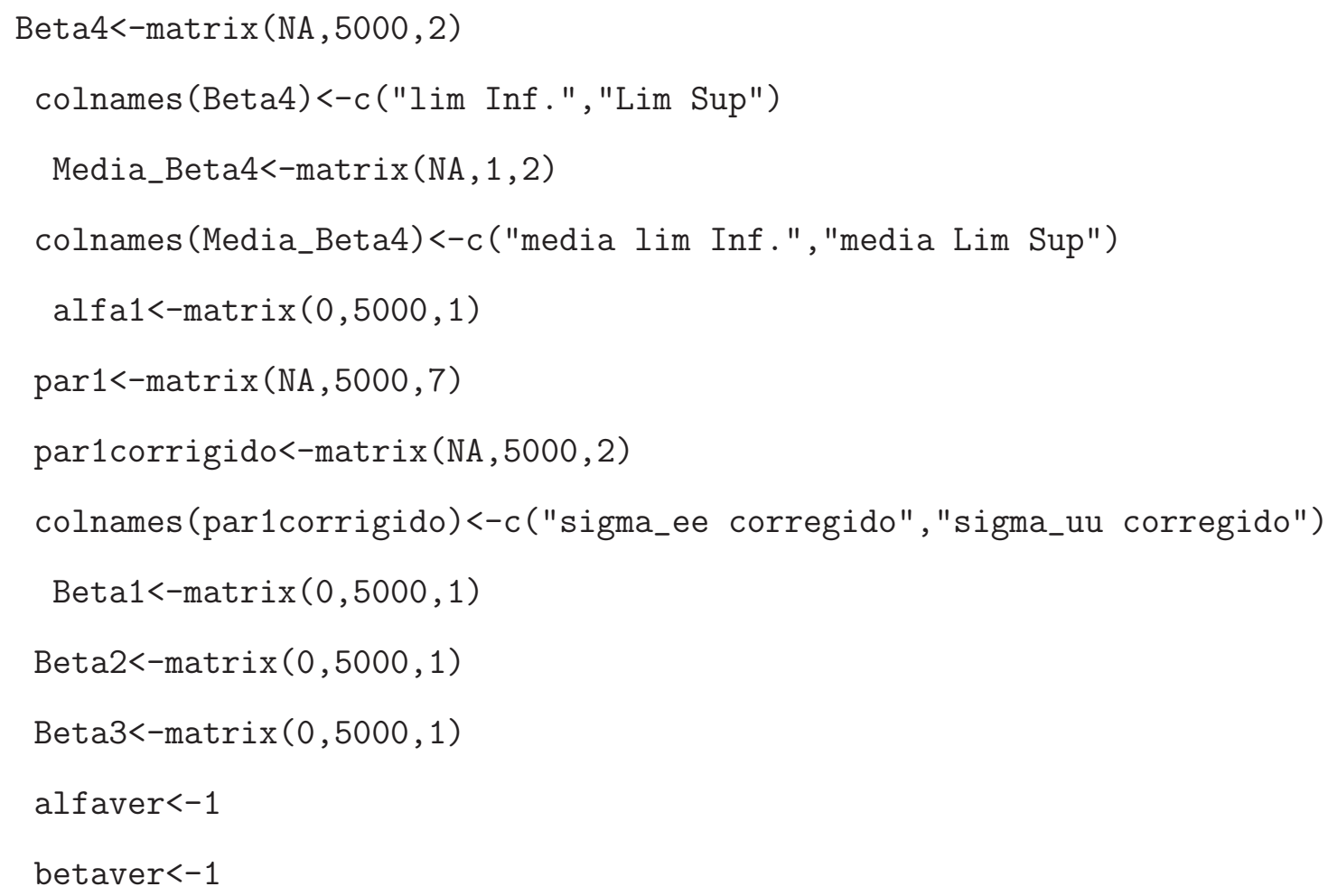




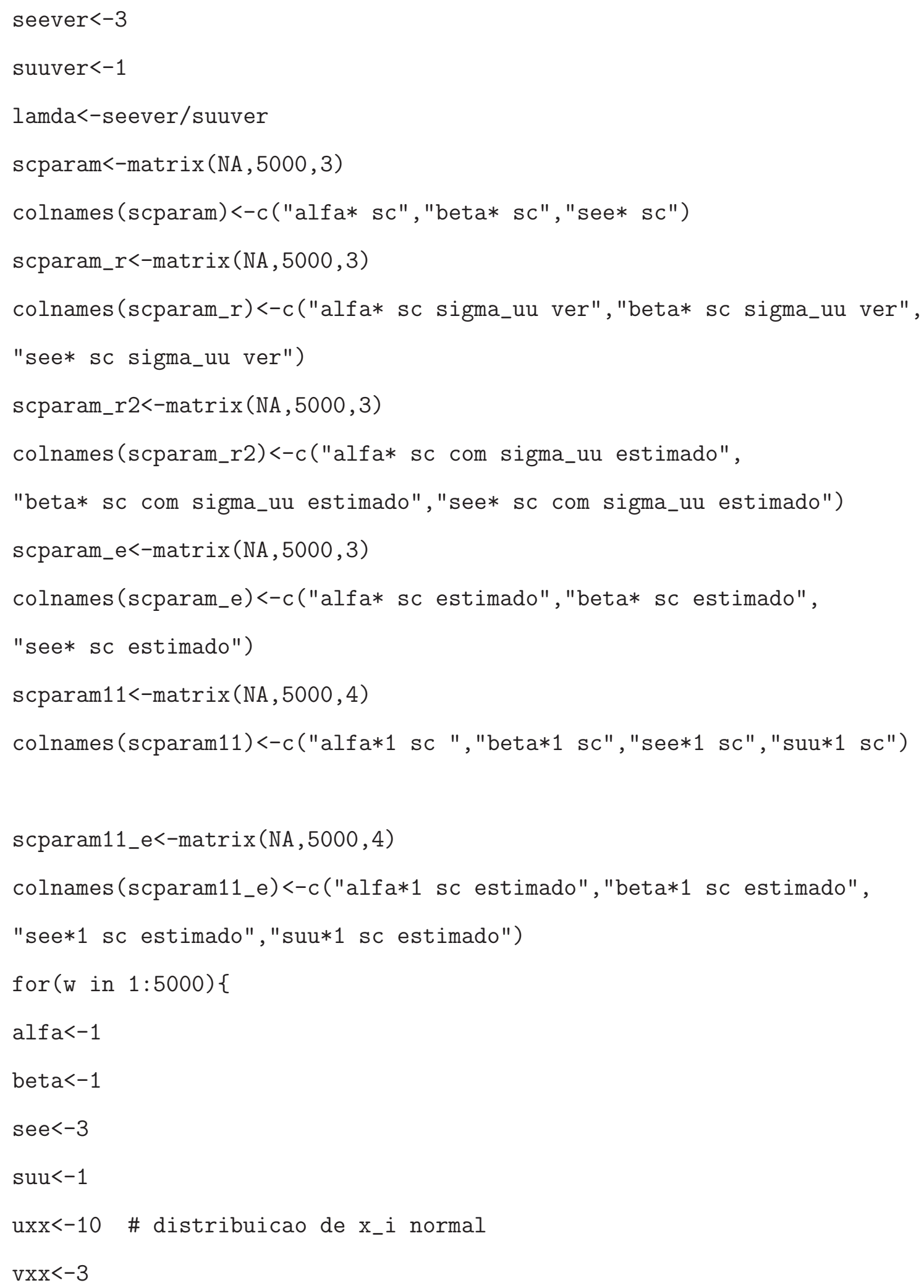




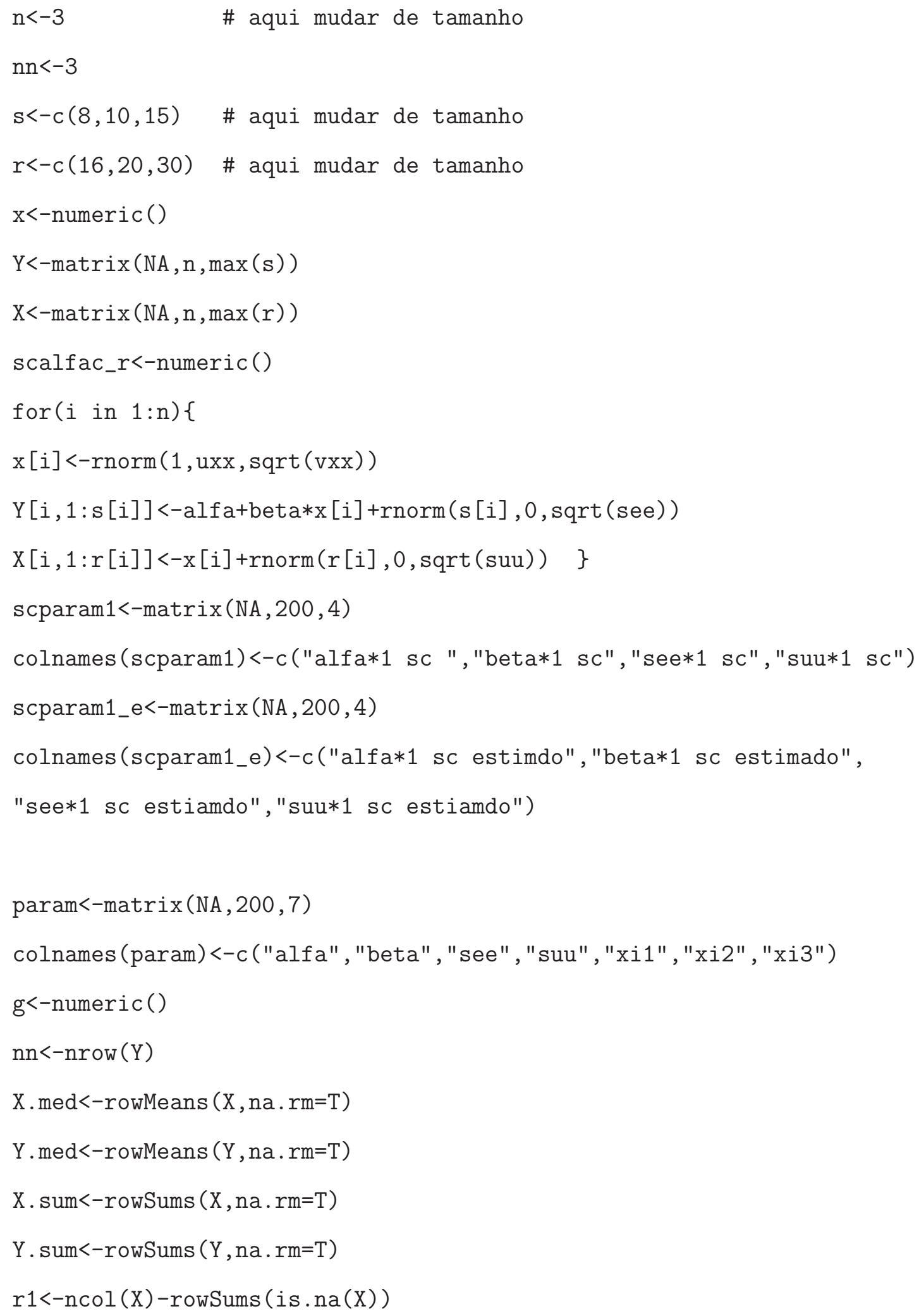




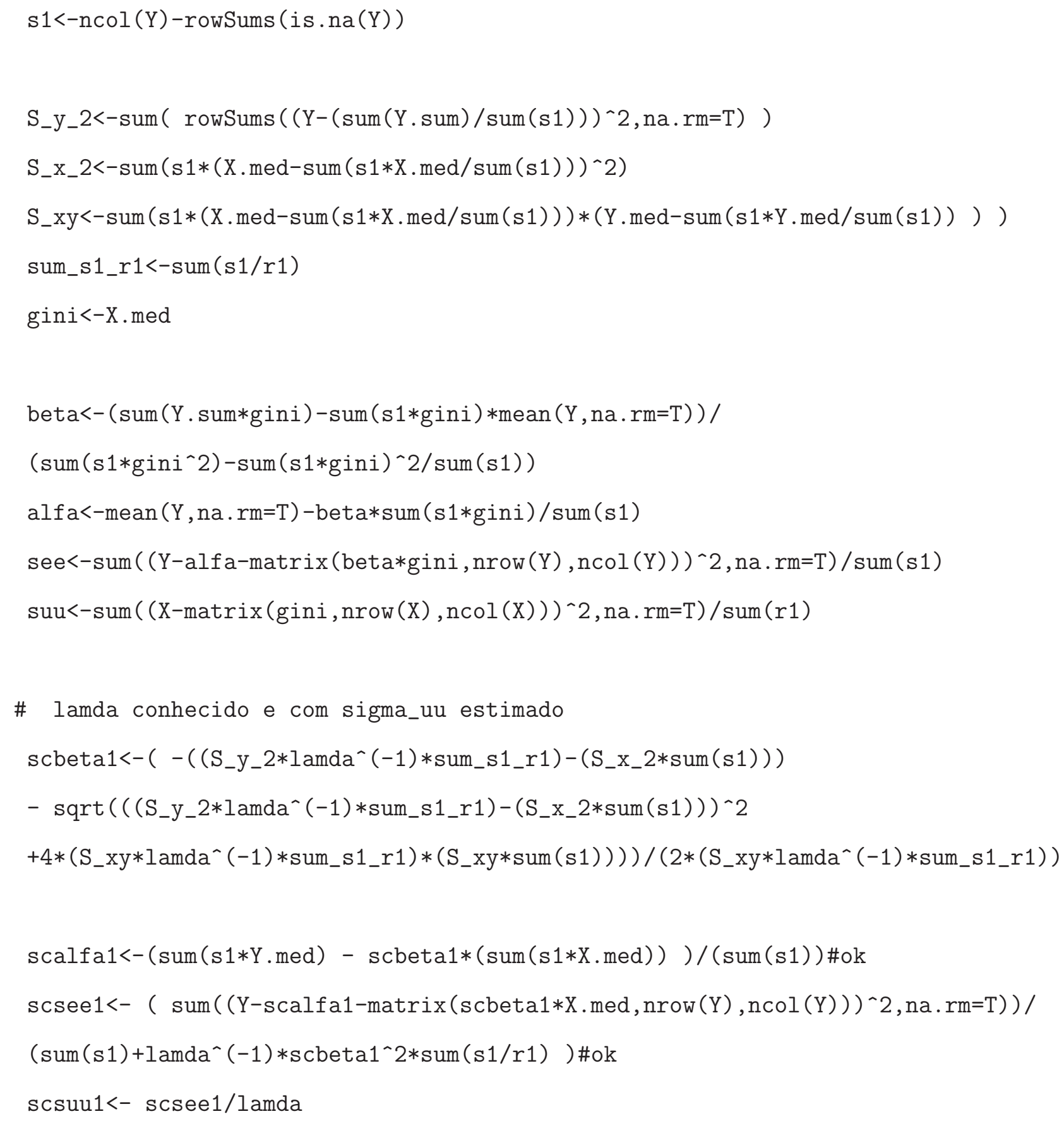




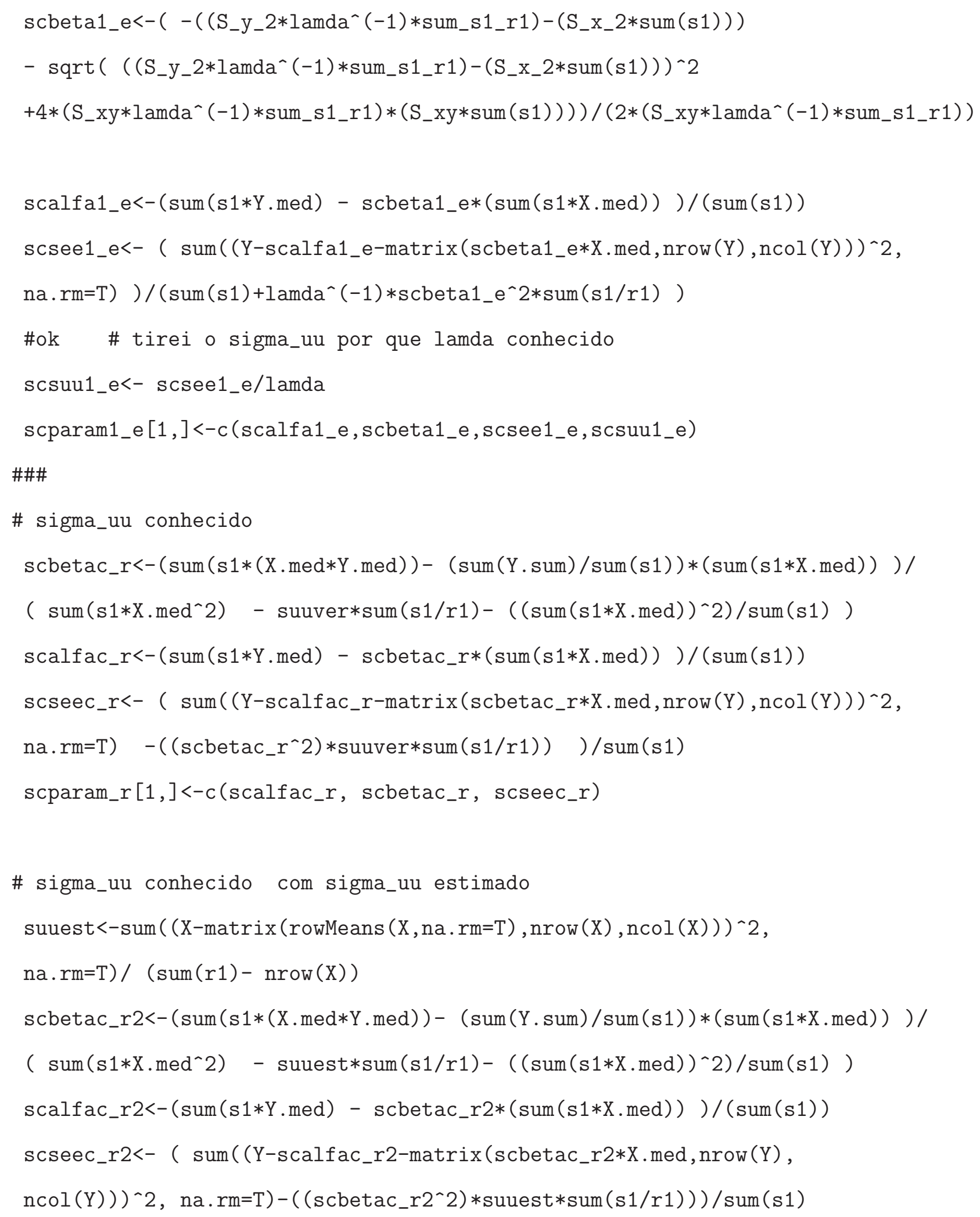


scparam_r2[1,]<-c(scalfac_r2, scbetac_r2, scseec_r2)

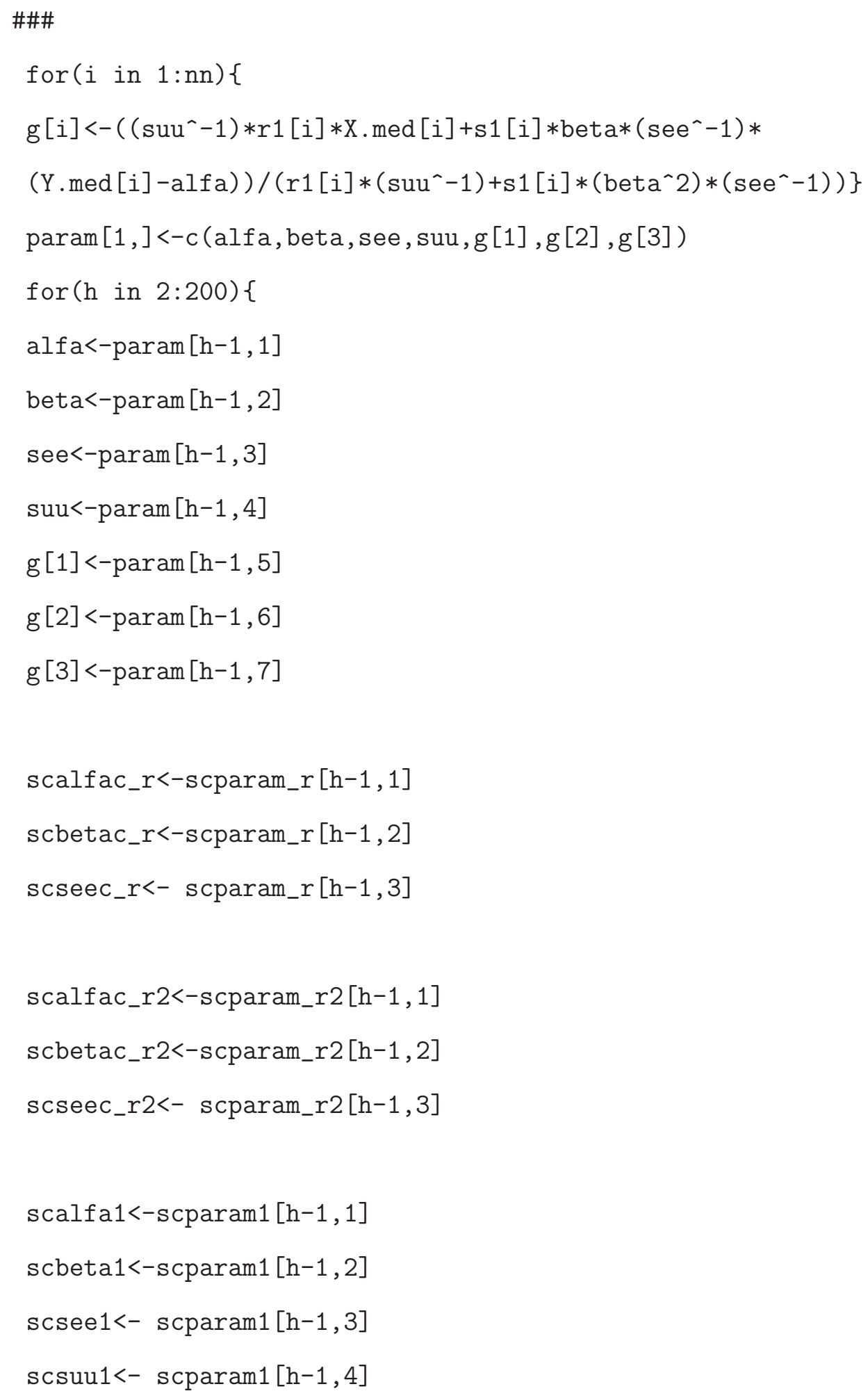




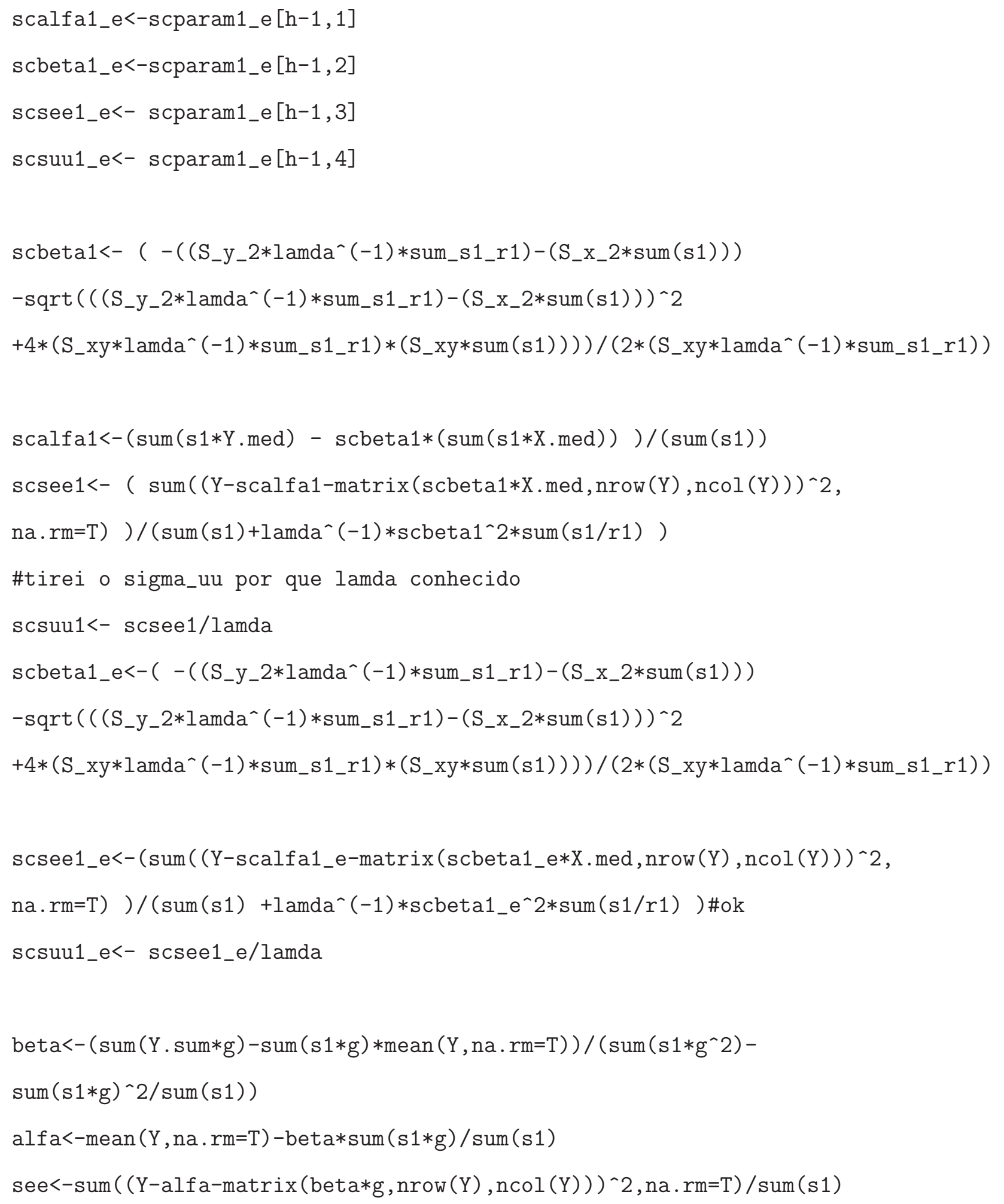




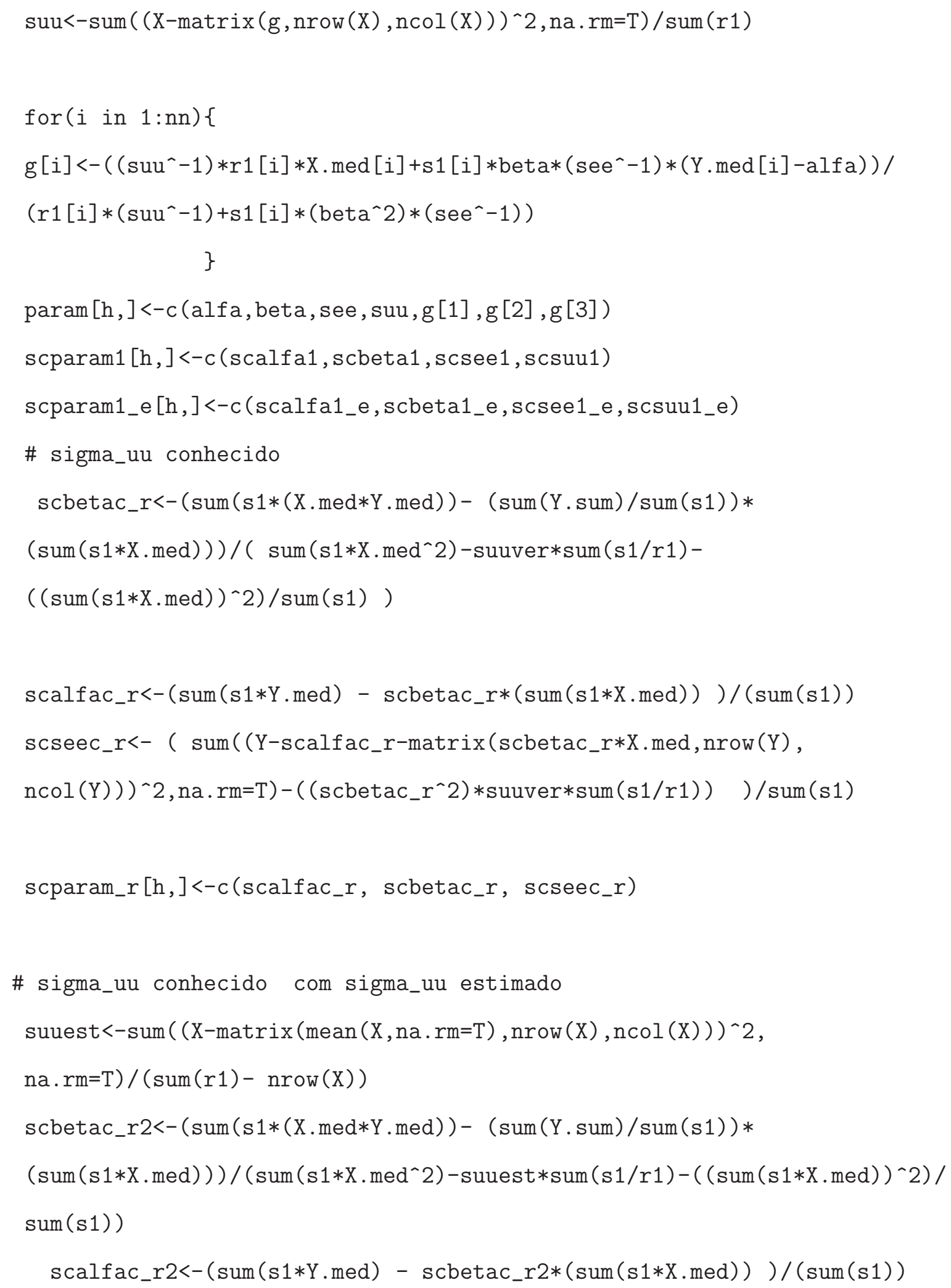




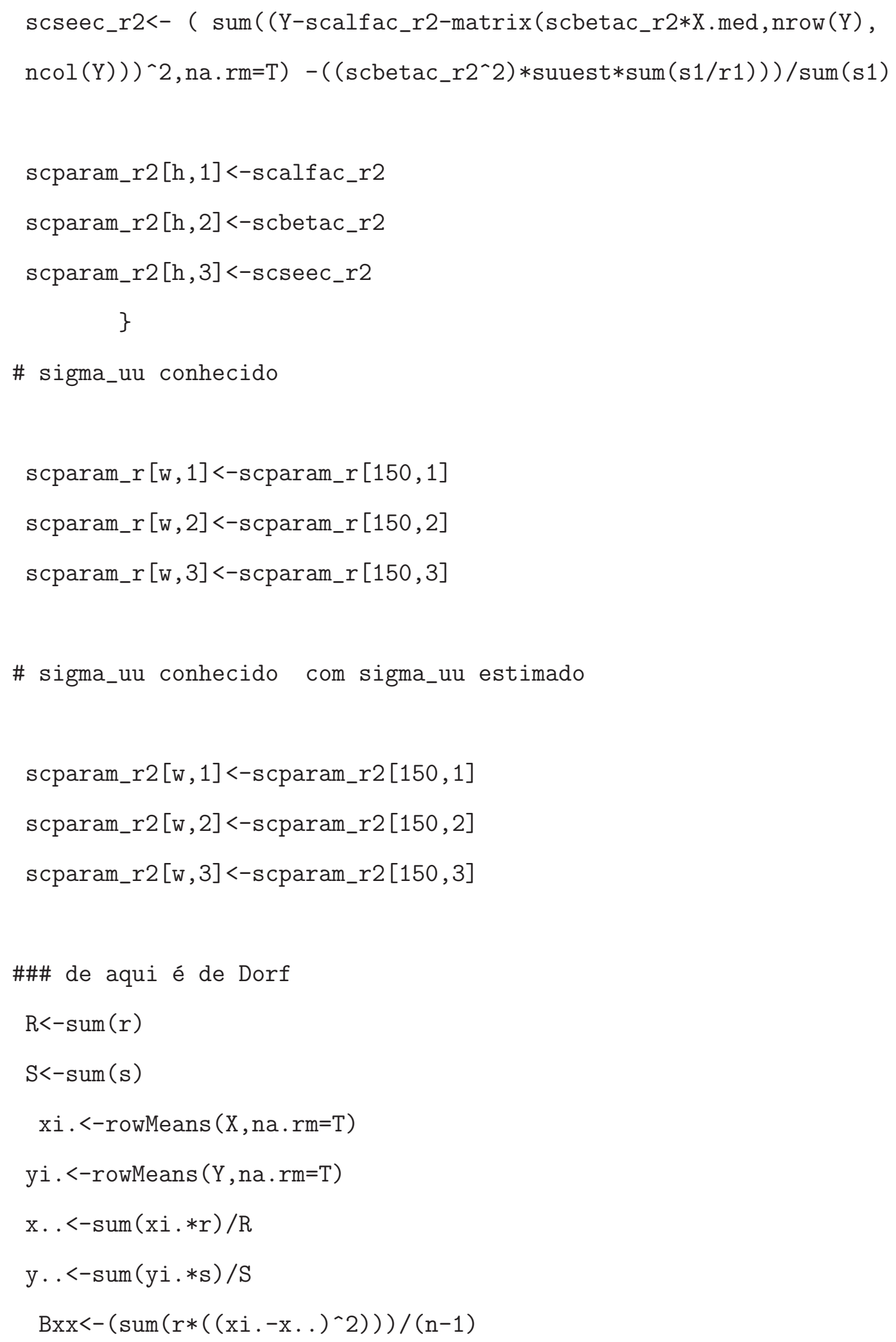




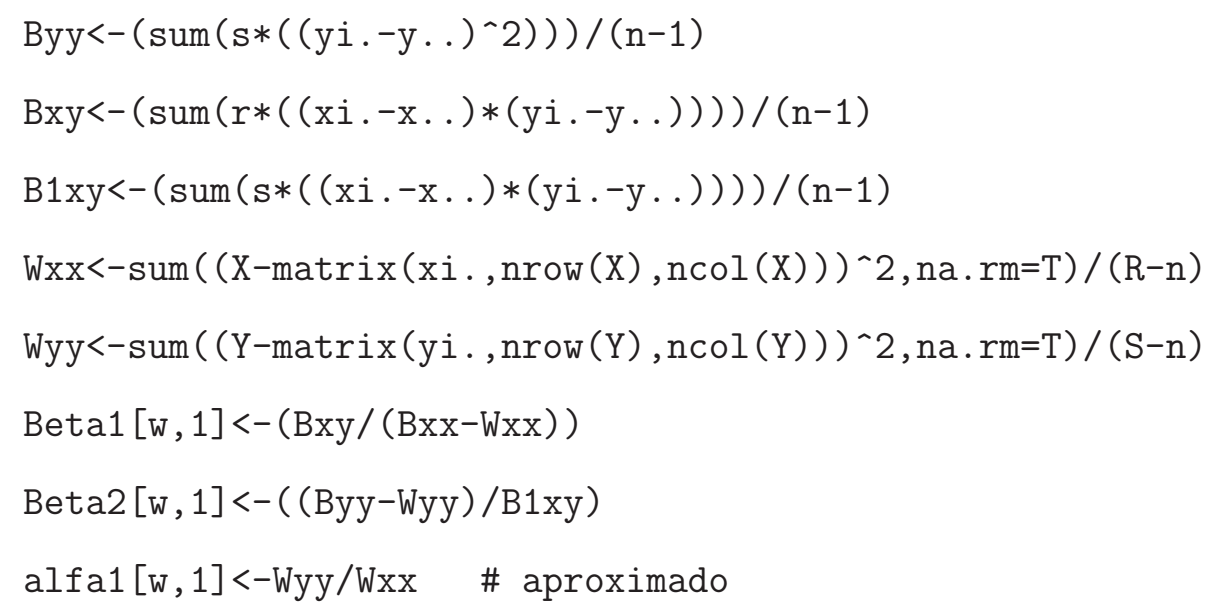




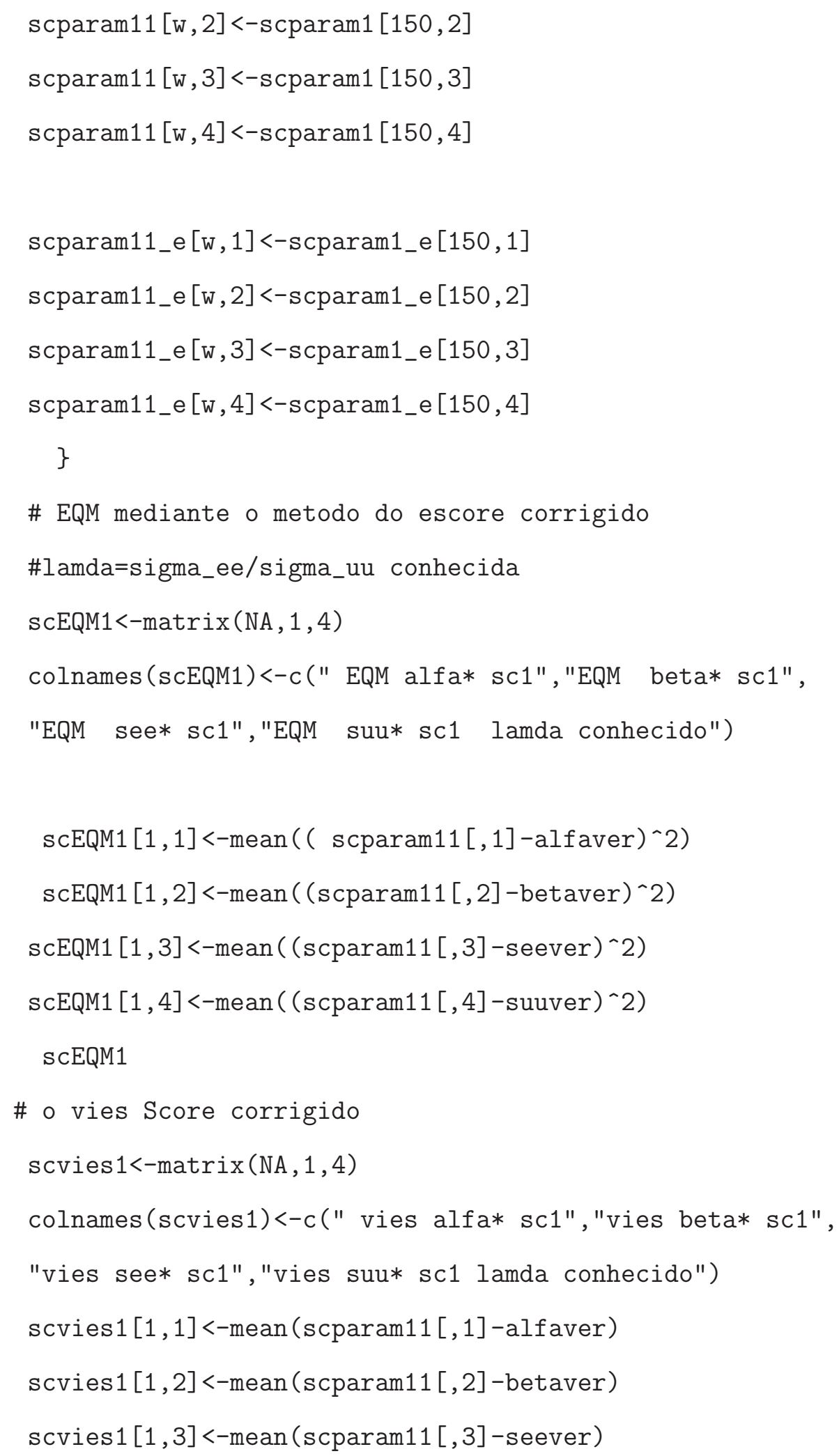




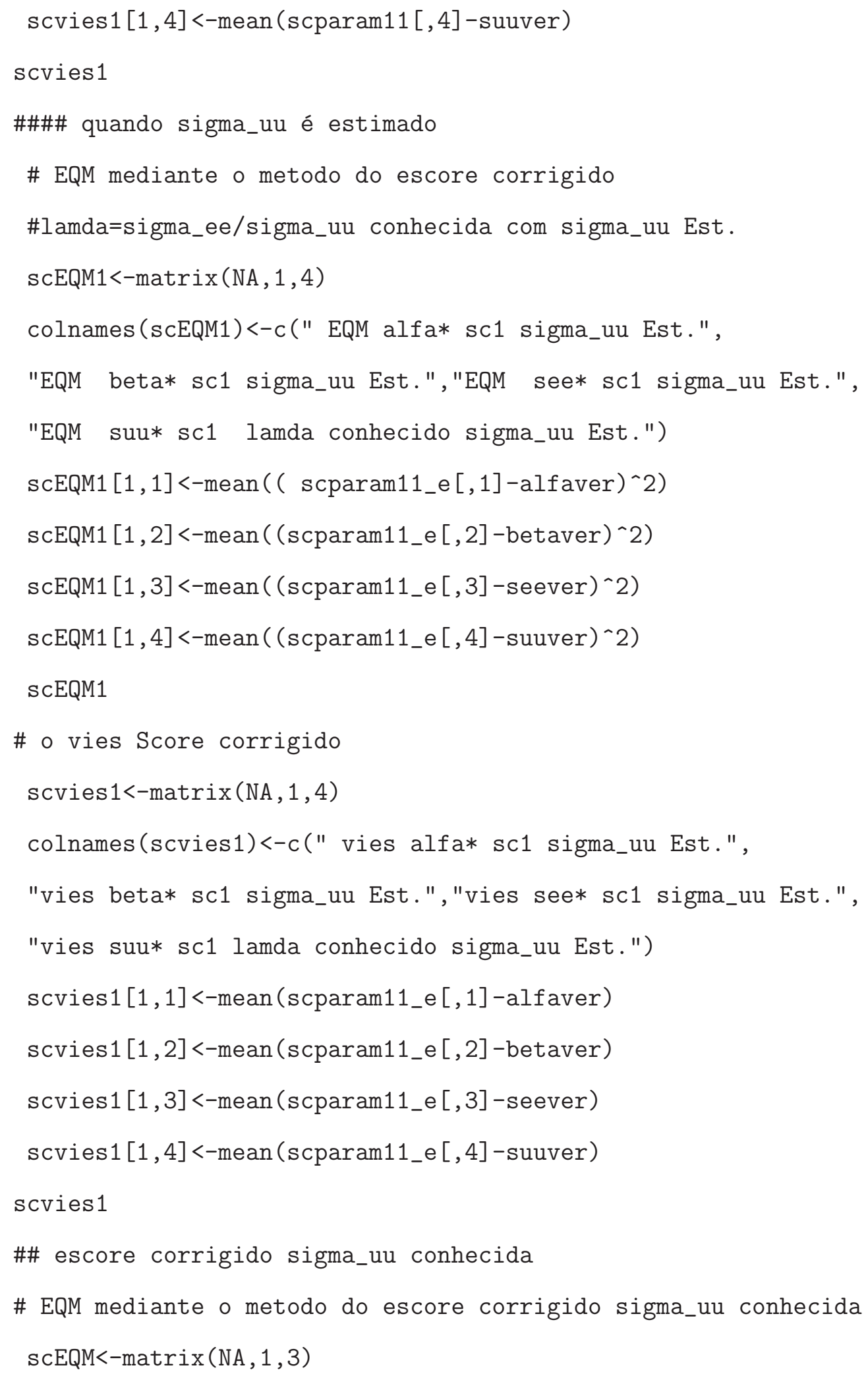




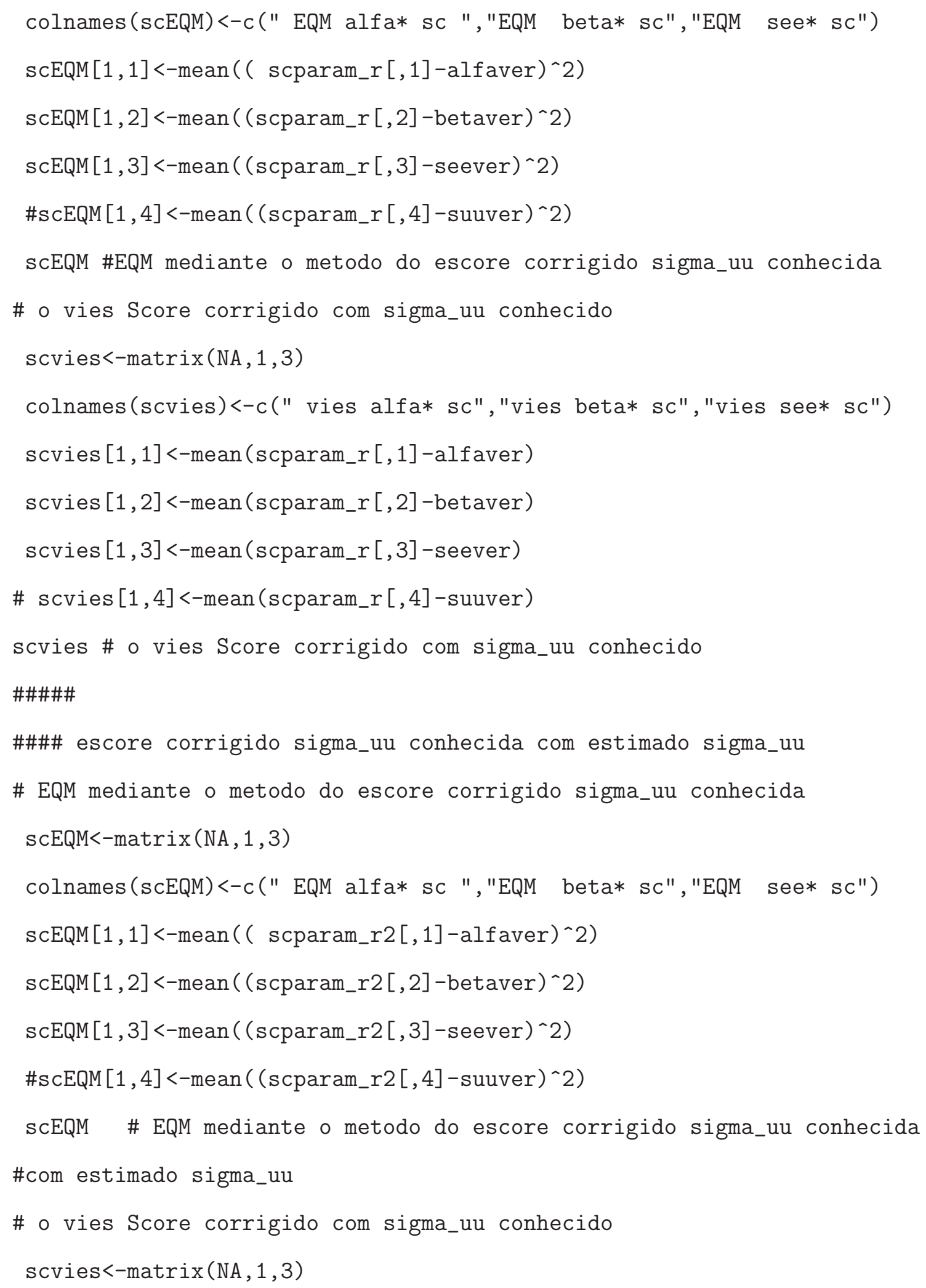




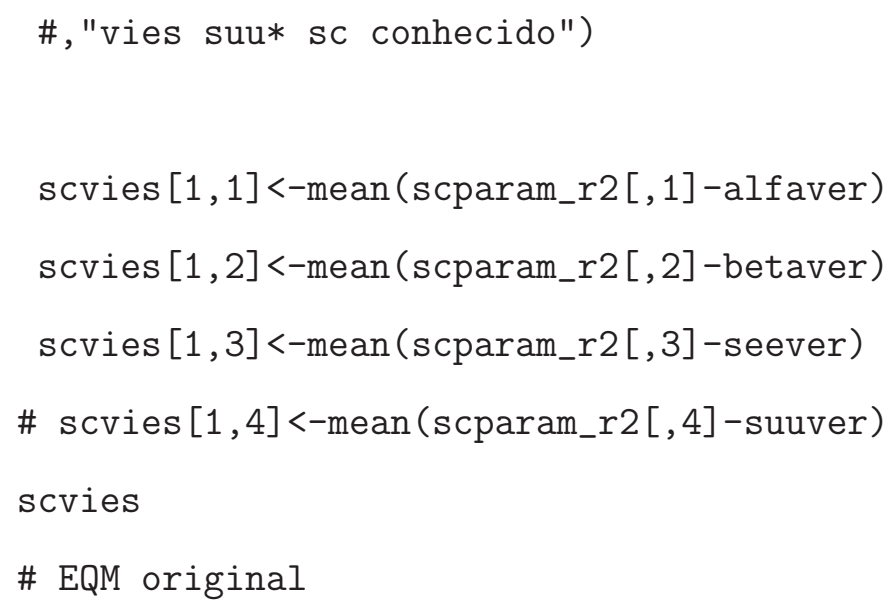




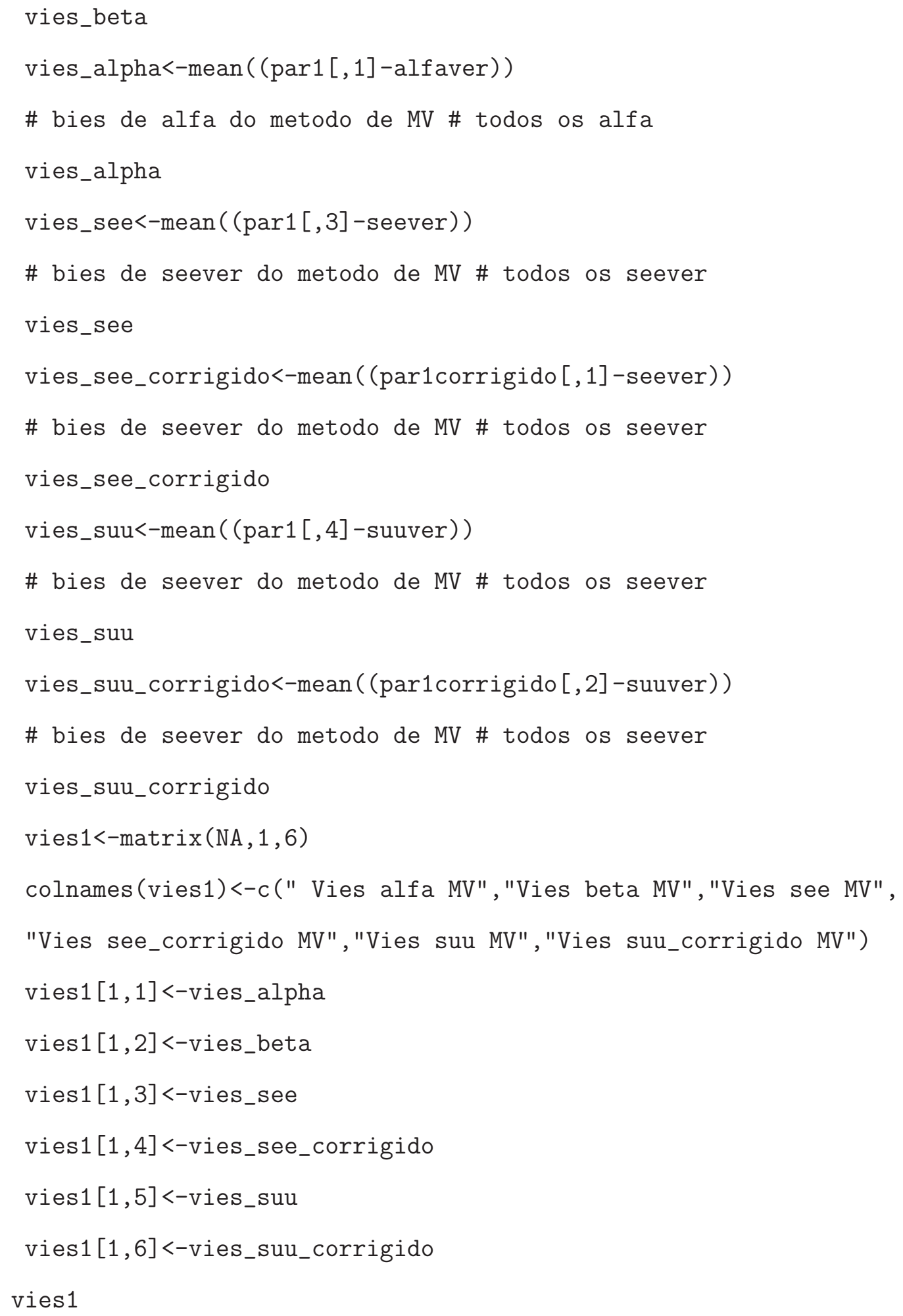




\section{Referências Bibliográficas}

Barnett, V. D. (1969). Simultaneous pairwise linear structural relationships, Biometrics, 25: 129-142.

Cheng, C. \& Van Ness, J. (1991). On the unreplicated ultrastructural model, Biometrika, 78: 442-445.

Dorf, M. \& Gurland, J. (1960). Estimation of the parameters of a linear functional relation, Journal of the Royal Statistical Society, B, 23: 160-170.

Fuller, W. A. (1987). Measurement Error Models, John Wiley and Sons.

Gimenez, P. C. (1997). Inferência em modelos com erros nas variáveis através do método do escore corrigido, $\mathrm{Ph}$. D. thesis, IME-USP.

Gimenez, P. C. \& Bolfarine, H. (1997). Corrected score functions in classical errorin-variables and incidental parameter models, Australian Journal of Statistics, 39: 325-344.

Gleser, L. (1985). A note on g.r.dolby's unreplicate ultraestructural error in variables model, Biometrika, 72: 117-124.

Gleser, L. J. (1981). Estimation in a multivariate error-in-variables regression model: large samples results, Annals of Statistics 9: 24-44. 
Hokama, J. (2001). Estimação e precisão no modelo de regressão linear com erros nas variáveis e mensurações replicadas, $\mathrm{Ph}$. D. thesis, IME-USP.

Hokama, J., Morettin, P. A., Bolfarine, H. \& Galea-Rojas, M. (2001). Consistent estimation in functional linear relationships with replications, Technical Report. Department of Statistics, University of São Paulo. 2001-20.

Kimura, D. (1992). Functional comparative calibration using em-algorithm, Biometrics, 48: 1263-1271.

Mak, T. K. (1982). Estimation in the presence of incidental parameters, Canadian Journal of Statistics, 2: 121-132.

Nakamura, T. (1990). Corrected score function for errors-in-variables models: Methology and application to generalized linear models, Biometrika, 77: 127-137.

Neyman, J. \& Scott (1948). Consistent estimates based on partially consistent observations, Econometrica, 16: 1-16.

Norden, R. H. (1973). A survey of maximum likelihood estimation, Rev. Inst. Internat. Statist., 41: 39-58.

Patefield, W. M. (1977). On the information matrix in the linear functional relationship problem, Applied Statistics, 26: 69-70.

Patefield, W. M. (1978). The unreplicated ultrastructural relation: Large sample properties, Biometrika, 65: 535-540.

R (2006). $\quad$ R Development Core Team: A Language and Environment for Statistical Computing, Vienna, Austria. ISBN 3-900051-07-0. Disponível em: http://www.R-project.org. 
Sen, P. K. \& Singer, J. M. (1993). Large Sample Methods in Statistics:An Introduction With Applications, Chapman and Hall, New York.

Serfling, R. J. (1980). Approximation theorems of mathematical statistics, New York : Florida State University.

Solari, M. E. (1969). The maximum likelihood solution of the problem of estimating a linear functional relationship, Journal of the Royall Statistical Society, $\boldsymbol{B}$, 31: $372-375$.

Stefanski, L. A. \& Carroll, R. J. (1987). Conditional score and optimal scores for generalised linear measurement error models, Biometrika, 74: 703-716.

Vilca-Labra, F., Arellano-Valle, R. \& Bolfarine, H. (1998). The maximum likelihood solution of the problem of estimating a linear functional relationship, Journal of Multivariate Analysis, 1 65:36-57. 